\title{
A review of Gallium Nitride LEDs for multi-gigabit-per-second visible light data communications
}

Sujan Rajbhandari' ${ }^{1,2}$, Jonathan J. D. McKendry ${ }^{3}$, Johannes Herrnsdorf ${ }^{3}$, Hyunchae Chun ${ }^{2}$, Grahame Faulkner $^{2}$, Harald Haas ${ }^{4}$, Ian M. Watson ${ }^{3}$, Dominic O'Brien², and Martin D. Dawson ${ }^{3}$

${ }^{1}$ School of Computing, Electronics and Mathematics, Coventry University, Coventry, CV1 2JH, United Kingdom

${ }^{2}$ Department of Engineering Science, University of Oxford, Oxford, OX1 3PJ, United Kingdom

${ }^{3}$ Institute of Photonics, Department of Physics, University of Strathclyde, Glasgow, G1 1RD, United Kingdom

${ }^{4}$ Institute for Digital Communications, Li-Fi R\&D Centre, University of Edinburgh, Edinburgh, EH9 3JL, United Kingdom

Abstract

The field of visible light communications (VLC) has gained significant interest over the last decade, in both fibre and free-space embodiments. In fibre systems, the availability of low cost plastic optical fibre (POF) that is compatible with visible data communications has been a key enabler. In free-space applications, the availability of hundreds of THz of the unregulated spectrum makes VLC attractive for wireless communications. This paper provides an overview of the recent developments in VLC systems based on gallium nitride (GaN) light-emitting diodes (LEDs), covering aspects from sources to systems. The state-of-the-art technology enabling bandwidth of GaN LEDs in the range of $>400 \mathrm{MHz}$ is explored. Furthermore, advances in key technologies, including advanced modulation, equalisation, and multiplexing that have enabled free-space VLC data rates beyond $10 \mathrm{~Gb} / \mathrm{s}$ are also outlined. 


\section{Introduction}

The evolution of computing, consumer electronics and mobile communications technologies is leading to an exponential increase in end-user data requirements. Reports by Cisco, for example, predict that there will be a nine-fold increase in mobile communications traffic from 2014 to the end of 2020, almost doubling every two years [1]. If the global traffic volume increases at the current rate, there will soon be 'a spectrum crisis' as radio frequency (RF) technology cannot keep pace with the demand [2]. It is also very unlikely that a single communication technology can support these growing data requirements in all places at all times. Hence, future generations of networks must support the co-existence and cooperation of different wireless technologies (RF, millimetre wave, and optical wave) [3]. With the evolution of the new wireless communication standards like the fourth generation $(4 \mathrm{G})$ and fifth generation $(5 \mathrm{G})$ networks, the available RF spectra are efficiently utilized by using advanced signal processing concepts such as massive multiple-input multiple-output (MIMO) systems and shrinking cell size leading to femtocells. In parallel, untapped frequencies at millimetre and nanometre wavelengths are also being considered. It is widely expected that visible light communications (VLC) systems and their extension to fully networked, bi-directional multiuser wireless systems referred to as $\mathrm{LiFi}$ ( see [4] for detail) will play a key part in 5G-and-beyond connectivity, especially for indoor environments [2], [5], [6].

Indoor lighting has also undergone a revolution with the recent advances in solid-state lighting (SSL) devices based on visible light emitting diodes (LEDs). Incandescent bulbs with an extremely low efficiency of 13-18 lumens per watt are being phased out. The compact fluorescent light (CFL) which was introduced in the 1990s offers a better energy conversion (55-70 lumens per watt). However, these efficiencies have recently been superseded by LED and laser diode (LD) based lighting. The average efficiency of Gallium-Nitride LEDs is higher than 100 lumens per Watt and is expected to reach 200 lumens per Watt by 2020 [7], [8]. Besides higher efficiencies, the LEDs also offer other advantages including a long operational lifetime (up to 50,000 hours), compact form factor, no emission of harmful ultraviolet or infrared radiation, mercury-free operation and a low maintenance cost. Additionally, the manufacturing cost of LEDs is dropping as the technology continues to mature for mass production, making LED based illumination economical in comparison to CFLs over its lifetime. Hence, the adoption of LED-based illumination is rising steadily [9], [10].

The popularity of SSL-based illumination has created a unique opportunity as each light bulb can potentially serve as a communication hotspot. Unlike traditional lighting devices, SSL devices can be modulated at a rate imperceptible to the human eye. This has paved the way for the dual function of illumination and communication using LEDs, in principle enabling communication at minimal extra cost and energy consumption [11]. The wide availability of lighting infrastructure and the feasibility of achieving communication rates beyond 100's of Mb/s makes VLC a cost effective and attractive complementary technique to RF technology.

The first reported VLC system based on a visible LED was in 1999 [12] when Pang et al. used modulated LED traffic lights for broadcasting audio and other information. Tanaka et al. adopted LEDs for illumination and communication in the early 2000s [13]. Interest in VLC now is growing rapidly with a number of practical demonstrations. In Japan, the visible light communication consortium (VLCC) [14] was formed in 2003 which is now superseded by the visible light communication association (VLCA) [15]. A number of key technologies were demonstrated in the European Union funded 'hOME Gigabit Access' project (OMEGA) project [16]. Recently, the UK government funded Ultra-parallel visible light communications (UP-VLC) [17] project has demonstrated a $10 \mathrm{Gbits} / \mathrm{s}$ VLC system [18]. In parallel to these research activities, there have been efforts to establish a VLC standard and IEEE802.15.7 was proposed in 2011 [19], [20]. A task group on shortrange optical wireless communication (OWC) was formed in 2015 to revise the IEEE 802.15.7 standard [21].

Early VLC demonstrations were based on conventional chip, broad-area $\left(0.1-1 \mathrm{~mm}^{2}\right)$ Gallium Nitride $(\mathrm{GaN})$ LEDs originally developed for lighting, which enabled data rates typically up to $100 \mathrm{Mbit} / \mathrm{s}$ [22]. Recently, there has been work on increasing the bandwidth of these devices by developing LEDs with dimensions of less than $100 \mu \mathrm{m}$ (' $\mu$ LEDs') [23]. These $\mu$ LEDs can be driven at a significantly higher current density than the broad-area LEDs and, hence, they offer an optical bandwidth in excess of several hundred $\mathrm{MHz}$ [24]. This is an order of magnitude higher than the traditional broad-area LEDs and makes $\mu$ LEDs attractive sources for multi-Gbit/s VLC systems [25], [26]. The illumination levels required for lighting can be achieved by operating multiple micro-LEDs in a ganged fashion or by a hybrid approach where micro-LEDs are used in conjunction with high-power LEDs.

Furthermore, the communication capacity can be increased by adopting multi-LED systems and applying techniques such as a) wavelength division multiplexing (WDM) and/or b) spatial modulation/multiplexing. In WDM, LEDs with different colours (commonly red-green-blue) carry independent data streams and the system relies on the successful separation of these colours at the receiver [18], [27]-[31]. Spatial modulation/multiplexing depends on the spatial separation of multiple transmitters and receivers to allow 
multiple streams of parallel data channels to be transmitted simultaneously. Several demonstrations of spatial multiplexing (also known as multiple input multiple output (MIMO)) systems have been reported with high spatial density [32] and data rates up to $7 \mathrm{Gbit} / \mathrm{s}$ [33].

In parallel with above-mentioned progress in LED manufacturing and multiplexing techniques, there has also been a significant advance in modulation schemes and signal processing. The initial demonstration of VLC systems adopted on-off keying (OOK) modulation [22]. Though the implementation of OOK is simple, because of low spectral efficiency ( $1 \mathrm{bits} / \mathrm{s} / \mathrm{Hz})$ it severely limits maximum achievable transmission speed. As a result, a number of multi-level advanced modulation schemes exploiting multiple dimensions (wavelength, polarisation and spatial) that VLC systems naturally possess, have been studied. The multi-level pulse amplitude modulation (PAM) exploits the amplitude of optical signals whereas orthogonal frequency division multiplexing (OFDM) exploits the frequency. Modulation schemes to utilize the phase, spatial and colour dimensions such as carrierless amplitude and phase (CAP) [34], optical spatial modulation (OSM) [35] and colour shift keying (CSK) [36], respectively, have also been significantly studied. As a result of these developments, VLC systems can now achieve transmission rate close to the Shannon capacity [37] and a $10 \mathrm{~Gb} / \mathrm{s}$ link has been practically demonstrated using LEDs [18], [34].

For applications where LEDs are used for both illumination and data communication, it is highly desirable to have a large-bandwidth LED source. Traditional rare-earth doped Yttrium Aluminium Garnet (e.g. Ce:YAG) phosphor-based white LEDs have a bandwidth of few MHz, limited mostly by the slow phosphor temporal response. In order to improve the bandwidth, it is necessary to either filter out the light from the slow phosphor at the receiver, or to replace the slow phosphor by a colour converter (or converters) with a fast response. It has been shown, for example, that organic colour converters can have a bandwidth in excess of 100 $\mathrm{MHz}$ and are suitable for white light generation [26], [38]. Using a "cool" white light generated by such colour converter, a data rate of $1.6 \mathrm{Gbit} / \mathrm{s}$ was demonstrated at an illumination level of $240 \mathrm{lux}$, [26]. which is the fastest data rate achieved so far with a single white light LED source at this lux level.

In this paper, a survey of the technologies that have enabled multi-Gbit/s VLC systems is given. The survey focuses mostly on the practical VLC demonstrations using GaN LEDs and the underpinning technologies that have enabled these demonstrations. The rest of the paper is organized as follows: an overview of a typical VLC system is given in Section 2. Section 3 introduces GaN-based LEDs and approaches to increase the modulation bandwidths and data rates using these sources. Section 4 surveys high-speed data communication demonstrations, outlining key technologies including modulation schemes, equalisation techniques, spatial multiplexing, and WDM. Finally, the conclusions and future outlook are presented in Section 5.

\section{An Overview of VLC systems}

A VLC system is enabled by modulating a visible light-emitting device, such as an LED or LD, using a data source. This modulated light propagates over a channel, such as free-space or a waveguide link, and the data is subsequently recovered using an optical sensor as shown schematically in Figure 1. The transmitter consists of a driver circuit to drive an Electro-Optical (E-O) converter such as an LED or LD. VLC systems normally employ light emitters such as an LED or LD with a diffuser which provides uniform illumination and are relatively safe to look at with the naked eye. Hence, intensity modulation (IM) is the only practical and costeffective method to modulate these light sources as the coherent communication requires a narrow linewidth coherent source (which conflicts the requirement for a good colour rendering index), and expensive external modulator and local oscillator. Optical coherent modulation and demodulation incur a significant cost, making them impractical for most of the targeted VLC applications. The receiver consists of an optics system, an OptoElectrical (O-E) converter such as a photodiode, amplifier(s) and signal processing circuitry. These elements are described briefly in the following section.

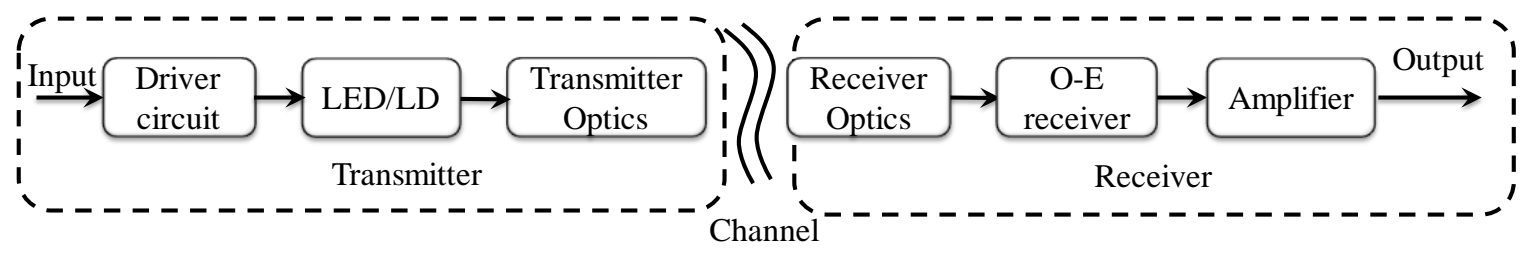

Figure 1: A block diagram of a VLC system. 


\subsection{Optical emitters}

White light suitable for illumination may be generated by a) combining a solid-state device (SSD) emitting light of one colour with a complementary colour-converting material b) or by combining the emission of multiple SSDs each emitting at different wavelengths. In the former approach, the emission from an ultraviolet or blue source (generally a GaN/InGaN blue LED/LD) is frequency down-converted using organic or, more typically, inorganic colour converters. In the second approach, emission from at least three monochromatic sources (red, green and blue) is spatially superimposed. The first approach using colour converters is commercially cheaper for mass production, where blue-emitting LEDs combined with a yellowemitting Ce:YAG phosphor is currently the dominant format of white-emitting solid-state lighting. The multicolour approach is more expensive but offers the flexibility of colour tuning and WDM for communications.

\section{a) Inorganic LEDs and LDs}

The peak emission wavelength of a SSD source depends on the bandgap energy of the p-n junction. LEDs fabricated from the indium gallium nitride ( $\mathrm{InGaN}$ ) alloys are the dominant form of semiconductor used in white light generation. These materials are direct bandgap semiconductors, capable in principle of producing light-emitting devices emitting light in the deep ultraviolet (UV) and visible regions of the electromagnetic spectrum. Commercial white light LED bulbs generally employ an InGaN LED coated with cerium(III)-doped yttrium aluminium garnet (Ce:YAG) phosphor. The Ce:YAG emits a broad yellow spectrum when excited with blue light. The white light appropriate for illumination is generated by mixing the blue electroluminescence (EL) from the SSD light source and the yellow phosphorescence from the phosphor [39], [40]. The yellow phosphor based illumination is compact and cost-effective. However, the yellow phosphor has a long upperstate lifetime, causing a possible bottleneck for VLC applications as the bandwidth of phosphor coated LED (PC-LED) is limited to a few $\mathrm{MHz}$ [22]. Alternative materials for colour conversion with bandwidth significantly higher than Ce:YAG have been demonstrated (see Section 4.6 for detail). However, the bandwidth of the broad-area blue LED itself is limited to 10's of $\mathrm{MHz}$ only. Hence, LD based illumination and communication has been considered recently [41]-[43]. LDs tend to have narrower spectral linewidth and higher modulation bandwidth than LEDs because the stimulated emission process reduces the carrier lifetime. However, there is a strict requirement for the LDs to be eye-safe, LDs have poor colour rendering due to their narrow linewidth, and require a complex driver circuit with temperature stabilization.

White light generation using the mixed emissions from three (RGB) or more monochromatic sources offers an excellent colour rendering index (>95) [39] and has potential to support high data rate using WDM. The manufacturing and maintenance cost of multi-colour devices, however, is higher and maintaining the desired colour balance is challenging as the individual sources are affected by temperature and aging effects. Moreover, the efficiency of AlInGaN-based LEDs begins to decrease as the peak emission wavelength moves into the green and yellow region of the visible spectrum, and while efficient orange and red-emitting LEDs can be fabricated from the Aluminium Gallium Indium Phosphide (AlInGaP-on-GaAs) alloy system, the lack of efficient green-yellow emitters poses a problem (often referred to as the 'green-yellow gap') [10]. The focus of this paper is on GaN-based VLC systems. Therefore, further discussion of GaN technologies will be given below.

\section{b) OLEDs}

OLEDs generate light using an organic thin film structure between anode and cathode. OLEDs have attractive attributes that make them suitable for display technologies. They are lightweight, flexible and cheap to manufacture as printing or spraying methods can be used. The downside of OLEDs for VLC applications is limited bandwidth, typically hundreds of kHz. OLEDs optimized for data transmission have been reported, with a bandwidth of up to $63 \mathrm{MHz}$, achieved through relatively small active areas of $0.018 \mathrm{~mm}^{2}$ in combination with specially chosen organic materials with carrier nobilities of $10^{-3}-10^{-2} \mathrm{~cm}^{2} / \mathrm{Vs}$ [44]. More generally, much low carrier nobilities in OLEDs severely limit bandwidths, and comparative motilities in carrier injection layers in typical InGaN/GaN LEDs are $>1 \mathrm{~cm}^{2} / \mathrm{Vs}$. With equalization (see Section 4.3), these devices are, however, feasible to use for low data rate applications as data rates higher than $10 \mathrm{Mbit} / \mathrm{s}$ have been demonstrated in a laboratory environment [45], [46].

\subsection{The VLC channel}

The free space VLC systems are either point-to-point or diffused links. In a point-to-point line of sight (LOS) link, a narrow beam transmitter communicates with a receiver having limited field of view (FOV). The point-to-point link supports a high data rate transmission due to low geometrical loss and being free from inter- 
symbol interference (ISI) due to channel path delay [47]. The LOS link, however, has a limited mobility and is susceptible to blocking and shadowing. Diffused links use wide divergence angle transmitters and large FOV receivers and do not rely solely on LOS path. As the result, the diffuse links do not require strict link set-up and do support mobility. However, the diffuse link suffers from high path loss and possible ISI.

The received optical power for a VLC link is related to the LOS channel direct current (DC) gain $H_{d}(0)$ and reflected path gain $d H_{r e f}(0)$ and is given by [48], [49]:

$$
P_{r}=\sum^{N_{L E D}}\left\{P_{t} H_{d}(0)+\int_{\text {Reflections }} P_{t} d H_{\text {ref }}(0)\right\}
$$

where $P_{t}$ and $P_{r}$ are transmitted and received optical powers, respectively and $N_{L E D}$ is the number of LEDs.

In general, lighting units installed on the ceiling or as desk lamps are used as the communication terminal. Hence, there is normally a direct LOS path between the transmitter and receiver. The diffused paths formed by the reflection of light from multiple surfaces including the walls, ceiling and floor can increase signal strength at the receiver. The contribution of optical power received by the diffused paths depends on a number of factors such as the room dimensions, the divergence angle, distribution and orientation of sources; the field of view, location and orientation of receiver and the reflectivity of the surfaces [50], [51]. Because of the path differences in the diffused channel, the received signal experiences a time delay causing ISI. However, the contribution of the diffused path for a VLC channel is relatively small, and ISI due to the multipath is not a serious issue except when the receiver is close to the wall [52]. There are a number of studies to fully characterize the path delay and possible effect on communication. As such channel studies are not within the scope of this paper, the interested reader can refer to references [47], [49], [53], [54].

Because of the low-loss transmission window in the blue and green regimes of step-index polymer optical fibre (SI-POF), guided-wave VLC has been considered for indoor applications [55]-[58]. The channel losses in SI-POF are relatively small in comparison to free space loss (e.g. $0.16 \mathrm{~dB} / \mathrm{m}$ at $650 \mathrm{~nm}$ and $450 \mathrm{~nm} 0.2$ $\mathrm{dB} / \mathrm{m}$ [55]). However, the limited bandwidth of the POF $(200 \mathrm{MHz} \times 50 \mathrm{~m})$ limits the achievable data rate for high-speed applications [55].

This survey mostly focuses on free-space communication. Nevertheless, the technology described in the paper can be easily adopted for POF as well, due to similarities in the way information is modulated and demodulated.

\subsection{Optical receivers}

The optical receivers are O-E converters which convert the received light to a photocurrent that can be further processed by the receiver system. An ideal optical receiver must have high sensitivity to light at the wavelength(s) used to transmit data, with high bandwidth and low complexity. Current VLC receivers are broadly based on photodiodes and image sensors. Charge-coupled device (CCD) or Complementary metal-oxide semiconductor (CMOS) based image sensors that are widely implemented in a number of modern devices (smartphone, camera, and laptop) can potentially be used as the VLC receiver. However, the image sensor has a low number of frames per second (fps) rate (in the $\mathrm{kHz}$ range or less), and hence they can support only low data rates in the kbits/s range [59]-[64]. Silicon photodiodes are more suitable for high-speed VLC applications as they have high external quantum efficiencies in the visible light range, and they are cheap and suitable for mass production. Among a number of families of silicon photodiodes, PIN photodiodes (with no internal gain) and avalanche photodiodes (APDs) are commonly employed for VLC applications. Because of the internal gain, an APD offers better sensitivity in comparison to a PIN photodiode [55], [65], [66] at the cost of complex circuitry and higher reverse bias voltage requirements. Recently, receivers based on single-photon avalanche diodes (SPADs) have also been studied for VLC applications [67]-[70]. Though SPADs based receivers can potentially have a higher sensitivity, the achievable data rate at present is limited to 100 's of $\mathrm{MHz}$.

\subsection{Advantages of VLC systems}

Besides being a 'green' communication technology by its further use of existing lighting infrastructure, VLC systems offer the following advantages:

\section{a) License-free operation and virtually unlimited bandwidth}

The RF spectrum is limited and heavily regulated throughout the world. Typically, license fees need to be paid to central governments in order to use part of the RF spectrum [71]. For example, UK mobile operators paid $£ 80.3 \mathrm{~m}$ to utilise a $900 \mathrm{MHz}$ band of frequency and $£ 119.3 \mathrm{~m}$ for $1800 \mathrm{MHz}$ in 2015 [72]. Other regions of the spectrum are also heavily regulated and allocated for restricted applications such as military or satellite use 
[73]. Moreover, the available spectrum is becoming scarce as new devices and technologies are being introduced. VLC systems, on the other hand, use the visible range of the electromagnetic (EM) spectrum from $370 \mathrm{~nm}$ to $780 \mathrm{~nm}$ which is readily available everywhere to operate free of cost without being constrained by regulations. Moreover, the communication bandwidth available in the visible range is $>300 \mathrm{THz}$ which is 10,000 times more than the entire RF spectrum which covers only $300 \mathrm{GHz}$ (see Figure 2).

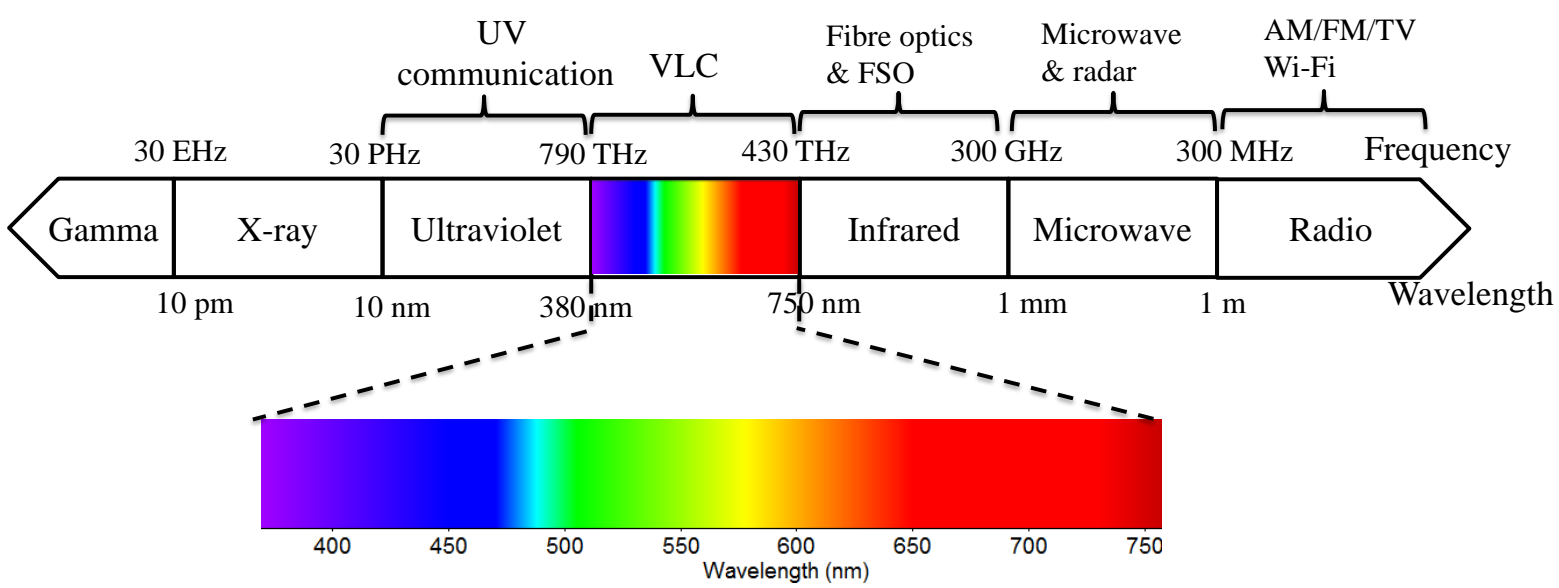

Figure 2: The frequencies and wavelengths of the electromagnetic spectrum. Also shown is the frequency allocation for different communication applications. FSO: free space optics, Wi-Fi: Wireless Fidelity, TV: television, AM: amplitude modulation, FM: Frequency modulation.

\section{b) High spatial reuse}

The optical spectrum cannot penetrate opaque objects including walls. This makes the coverage of a VLC system limited to, e.g., one room. As a result, the same spectrum can be reused in other rooms. Moreover, the direction and coverage of optical emission can be precisely controlled using optics, enabling dense space division multiplexing with high capacity per unit volume.

\section{c) Ultra-dense spatial multiplexing}

In order to provide uncorrelated copies of the signal for successful MIMO decoding, the minimum separation of the adjacent transmitter and receiver elements must be greater than half the carrier wavelength [74]. This limits the adjacent transmitter/receiver separation to the centimetre range for $\mathrm{GHz} \mathrm{RF}$ carriers and higher for MHz carriers. The theoretical spatial separation for optical MIMO (using visible wavelengths), on the other hand, is in the hundreds of nanometre range, and has been demonstrated practically using displays and charged couple devices (CCDs) with pitch sizes of micrometres [32], [33], [75].

\section{d) Security}

Because of confinement of the optical spectrum within a room, VLC systems intrinsically provide a physical layer security against eavesdropping. Moreover, the data transmission is available only in the illuminated area, and hence the VLC system is 'visibly secure'[76].

\section{e) Green communication}

VLC uses the existing lighting infrastructure and highly energy-efficient LEDs for data communication. Since there are minimum illumination requirements in home and office environments, most of the energy LEDs used are for illumination. Communication is an additional benefit that was not envisaged when LED-based lighting was developed. As the LEDs are deployed in many new areas including illumination, signalling, smartphone, consumer electronics, toys and clothing/wearables, VLC is expected to be a key technology in realising the so-called Internet of Things (IoT) [77]-[79]. 


\section{Gallium Nitride-based LEDs}

\subsection{Introduction to GaN LEDs}

As described above, the development of III-nitride LEDs over the last 20 years has made available solidstate optoelectronic emitters that span the entire visible spectrum. III-nitride LEDs with reported external quantum efficiencies (EQEs) in excess of $56 \%$ have been available for several years [10]. Ongoing efficiency improvements in industrial laboratories now focus heavily on techniques for enhancing light extraction efficiency, which at the same time are viable for mass production [80]. While internal quantum efficiencies are maximized for active regions containing multiple InGaN-based quantum wells (QWs) and emitting in the blueviolet spectral region, phosphor-based colour conversion and mixing, as already discussed, will allow LED lamps to supersede incandescent and fluorescent light sources as the dominant technology used for generalpurpose lighting [9].

Today, the vast majority of III-Nitride LEDs are grown using Metal Organic Chemical Vapour Deposition (MOCVD), where the LED epitaxial structure is grown on a single-crystal substrate [81]. The most commonly-used substrate material is sapphire $\left(\mathrm{Al}_{2} \mathrm{O}_{3}\right)$. Although sapphire does not have an intrinsically good lattice match to hexagonal wurtzite-phase GaN, it does offer matching of crystal symmetry, and sophisticated but highly reproducible growth initiation processes have been developed in MOCVD. Sapphire substrates are most commonly used in the (0001) or c-plane orientation, where hexagonal wurtzite-phase GaN grows with its unique crystallographic $c$-axis perpendicular to the substrate surface. The two alternative substrate materials likely to compete with sapphire in future commercial LEDs are silicon ( $\mathrm{Si}$ ) and bulk GaN. Si offers advantages in terms of low cost, ready availability of larger (200 mm diameter and larger) substrates, and compatibility with contemporary automated manufacturing lines. Bulk GaN currently remains expensive, and available only in smaller sizes. However, its use as a substrate greatly simplifies growth of device structures in non-(0001) orientations, as discussed further below, for which process windows for hetero-epitaxy may be prohibitively narrow.

The common use of an insulating sapphire substrate requires that both anode and cathode contacts are made on the same side of the device, in contrast to many other types of III-V semiconductor LED grown on conductive substrates. This is illustrated schematically in Figure 3, where it can be seen that the p-type layer and multi-quantum well (MQW) region have been etched through to allow a recessed electrical contact to be made to the n-type layer below. The same contact geometry applies to GaN LEDs grown on Si substrates, as nonconductive AlGaN-based buffer and strain management layers must be grown on the Si before the functional layers of the LED structure.

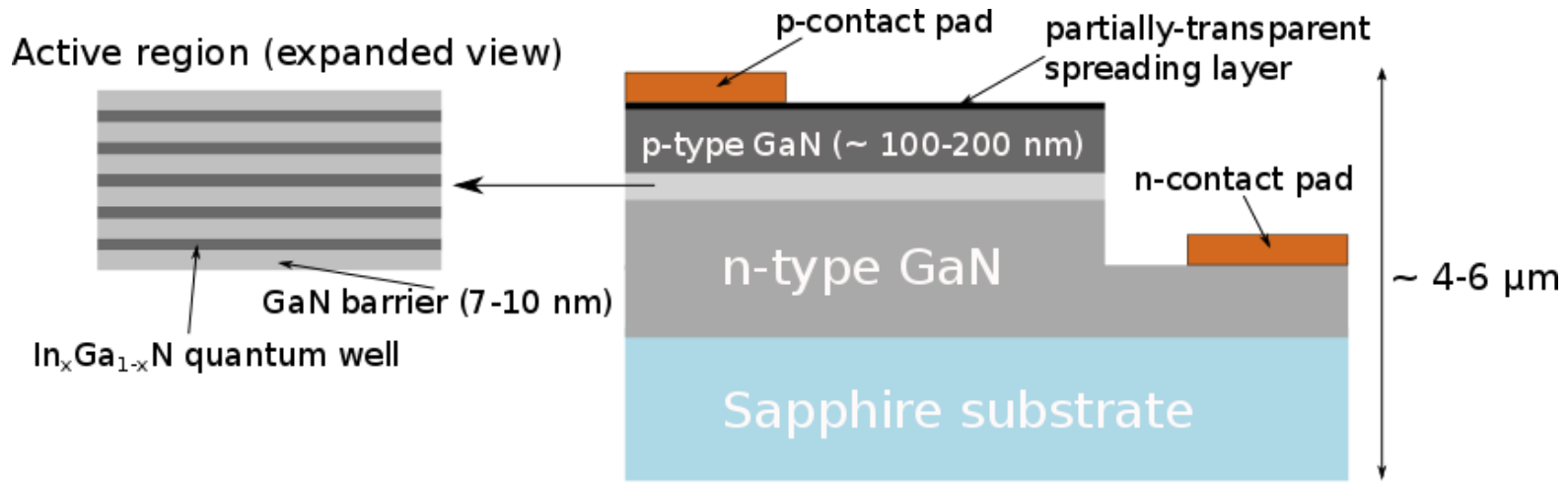

Figure 3 - A simplified cross-section of a typical III-Nitride LED, fabricated in a top-emitting geometry. Additional thin functional layers are generally present above and/or below the active quantum well region to control carrier injection and confinement.

All contemporary III-nitride LEDs have the characteristic that conductivity in the n-type layers of the device greatly exceeds that in the p-type region. Effective current spreading from the anode in the top-emitting geometry shown in Fig. 3 depends upon a transparent conductive layer deposited over the emitting mesa during device fabrication. This spreading layer can be either a sufficiently thin metal layer, or consist of a transparent conductive oxide. The transparency of sapphire substrates, however, also allows devices to be designed with light extraction exclusively through the substrate (so-called "flip-chip" configuration), allowing for a thick metal p-contact across the entire mesa region. The utility of such approaches is further enhanced by the possibility of fully removing the growth substrate during processing; distinct techniques for this exist for sapphire and Si.

Current commercial III-nitride LEDs are typically optimised for lighting or display applications, and their design is chosen to optimise parameters such as efficiency, output power and cost, rather than parameters 
of importance to VLC such as modulation bandwidth. The area of a typical rectangular-shaped LED die as shown in Figure 3 is several hundred microns per side, and many die may be connected together in series or in parallel in a single package, further increasing the effective active area.

This large area is chosen, in part, to mitigate the effects of the 'efficiency droop' phenomenon - where the internal quantum efficiency (IQE) of III-nitride LEDs peaks at relatively modest current densities (tens to hundreds of $\mathrm{A} / \mathrm{cm}^{2}$ ) and decreases at higher current densities [82]. Therefore, increasing the area allows the device to operate over its operating current range whilst in a regime where the IQE remains relatively high. An increase in the total area over which current is injected also alleviates the very significant engineering challenges in heat extraction, which is essential to keep commercial packaged devices within their design operating temperature range.

With such large areas, the modulation bandwidths of these LEDs are ultimately limited by their $R C$ (resistance $\times$ capacitance) time constant. Nonetheless, modulation bandwidths of the order of $20 \mathrm{MHz}$ are possible from a typical 'off-the-shelf' III-Nitride LED [83]. The small-signal capacitance of LEDs is still subject to investigation [84]but to our current knowledge the RC limit becomes significant above device sizes on the order of $200 \times 200 \mu \mathrm{m}^{2}$. The rest of this section will describe various work that has been undertaken to optimise the design of III-nitride LEDs for VLC, in particular how the modulation bandwidth can be increased by a variety of methods which are summarized in Table ITable.

Table I: Overview of approaches to increase the modulation bandwidth of GaN LEDs

\begin{tabular}{|c|c|c|}
\hline LED technology & Maturity & Bandwidth (MHz) \\
\hline High Power Broad area LEDs & Commercial mass production & 200 \\
\hline GaN RCLEDs & Academic prototypes & 120 \\
\hline Semi/non-polar GaN LEDs & $\begin{array}{c}\text { Academic and industrial research on } \\
\text { epitaxial growth }\end{array}$ & 1100 \\
\hline Bespoke GaN epitaxial structures & Purely academic research & $>400$ \\
\hline GaN Nano-wire LEDs & Purely academic research & 2000 \\
\hline GaN micro-LEDs & $\begin{array}{c}\text { Early stage of commercialization } \\
\text { (targeting niche-markets) }\end{array}$ & 200 \\
\hline Plasmonic LEDs & Academic prototypes & Prosper \\
\hline
\end{tabular}

\subsection{Approaches to increasing GaN LED bandwidth}

Resonant-cavity LEDs (RCLEDs)

Resonant-cavity light-emitting diodes (RCLEDs) are a form of LED in which the light-emitting region is located within an optical cavity, designed in such a way that the cavity is in resonance with the emission wavelength of the light-emitting region (hence the term, resonant cavity). A simplified cross-sectional schematic of an RCLED is shown in Figure 4(a). The active region of the RCLED, shown as a single QW in Figure 4(a), is located at the antinode of the standing optical wave of the cavity defined by the mirror layers. One of the mirrors would typically have a lower reflectivity than the other, such that light is predominantly emitted through that mirror, which acts as an output coupler.

The cavity has several beneficial effects: enhanced directionality, i.e. an enhancement of the light intensity emitted normal to the device surface; enhanced spectral purity; and higher extraction efficiency [85], [86], these properties make RCLEDs particularly attractive for fibre-based VLC, where the enhanced directionality and spectral purity respectively enhances coupling to fibres and reduces chromatic dispersion.

The mirrors used in RCLEDs can take various physical forms, and the two mirrors may be dissimilar in a given device. Where possible, it is advantageous if a distributed Bragg reflector (DBR) can be grown epitaxially before growth of the active region. Such an epitaxial DBR may be combined with a second non-epitaxial dielectric DBR, and this provides a straightforward way to obtain a very high- $Q$ cavity. Dielectric DBRs used in RCLEDs are usually formed by alternating layers of $\mathrm{SiO}_{2}$ and a transition metal oxide, such as $\mathrm{TiO}_{2}$ or $\mathrm{Ta}_{2} \mathrm{O}_{5}$. However, even interfaces between dissimilar materials pairs such as $\mathrm{GaN}$ and sapphire, or GaN and a metal contact, can suffice as one mirror in a RCLED with relatively low $Q$.

Challenges inherent in growing epitaxial DBRs mean that RCLEDs based on III-Nitride materials are not yet highly developed, and it is therefore relevant to mention relevant results obtained with other III-V materials. 
RCLEDs emitting around approximately $650 \mathrm{~nm}$ have emission which coincides with a window of low absorbance in POF, as can be seen in Figure 4(b). Compressively strained GaInP QWs were embedded within AlGaAs-AlAs Distributed Bragg Reflectors (DBRs) in these RCLEDs, for which modulation bandwidths of up to $200 \mathrm{MHz}$ were reported [87]. Similar AlInGaP RCLEDs are now readily available commercially. Pessa et al. reported $622 \mathrm{Mb} / \mathrm{s}$ transmission over $1 \mathrm{~m}$ of step-index POF, which indicates that data rates in the order of hundreds of megabits are achievable over POF using red-emitting AlInGaP RCLEDs [88].

RCLEDs emitting in the blue and green are also desirable for use as transmitters for POF-based communications, as the lowest absorbance losses in POF are found at these wavelength ranges (Figure 4). As already noted, epitaxial growth of suitable DBRs in the AlInGaN alloy system is challenging. The most obvious approach to growing III-nitride DBRs is to vary the AlN fraction in AlGaN layers, and Shaw et al. modelled RCLEDs with such DBRs explicitly for VLC applications with POF [89]. In the AlGaN system, however, only small refractive index differences can be achieved between adjacent DBR layers, and growth of more layers to increase reflectivity leads to severe strain management issues. These disadvantages of all-AlGaN DBRs led to extensive studies of AlInN-based DBRs, which have been applied in RCLEDs with one dielectric DBR [90]. AlInN can be lattice matched to $\mathrm{GaN}$ itself at an InN fraction of $\sim 18 \%$, and nominally strain-free DBRs can also be grown with various combinations of $\mathrm{AlGaN}$ and AlInN layers. Disadvantges of working with AlInN in MOCVD include the slow growth rates, and repeated temperature ramps required during DBR growth. To our knowledge, current applications of III-Nitride RCLEDs in optical communication links involve devices in which no epitaxial DBR growth was attempted. Data transmission at $200 \mathrm{Mb} / \mathrm{s}$ over $100 \mathrm{~m}$ of POF was reported by Akhter et al. using a RCLED with a peak emission wavelength of $509 \mathrm{~nm}$ [91], and Tsai et al. demonstrated 100 $\mathrm{Mb} / \mathrm{s}$ transmission over $100 \mathrm{~cm}$ of free space using a blue-emitting RCLED [86].

(a)

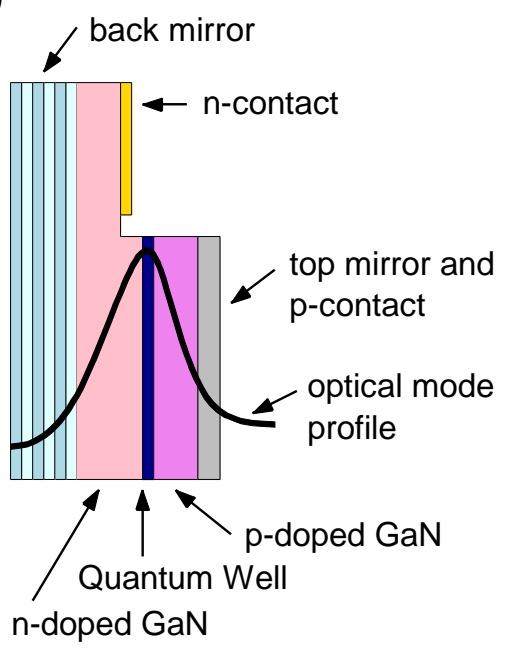

(b)

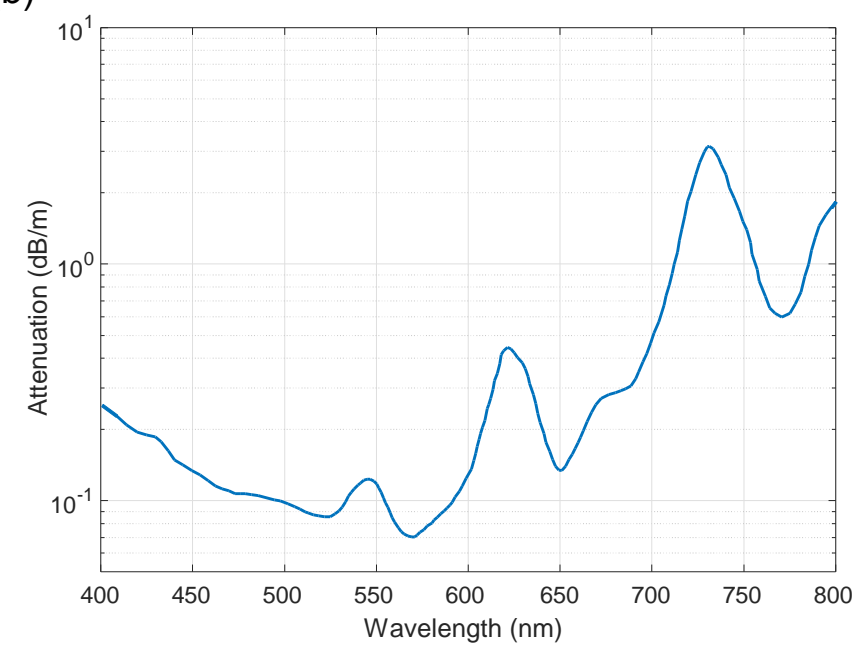

Figure 4: (a) simplified cross-sectional schematic of a resonant-cavity LED (shown oriented to emit horizontally) and (b) attenuation of step-index polymer optical fibre versus wavelength. Adapted from [92].

\section{b) Semi/non-polar GaN LEDs}

Nearly all GaN-based semiconductor epitaxial structures grown today have a hexagonal (wurtzite) crystal structure. As noted already, growth in an orientation with the unique $c$-axis of the wurtzite phase perpendicular to the substrate surface remains the most common, and was historically developed and optimized well before growth in other possible orientations. The growth orientation affects device properties not only through the obvious anisotropy in crystal structure, but also through the effects of polarisation fields discussed shortly, running parallel to the $c$-axis or [0001] crystal direction. Reducing deleterious effects of these fields has motived much recent interest in growth in non-traditional orientations. Figure 5 illustrates the most important growth orientations on hexagonal GaN, and Scholz [93] presents an overview of typical approaches to realize different growth orientations. Growth in semi-polar orientations has generally offered larger process windows than growth in non-polar orientations. Also growth in all the non-(0001) orientations often uses bulk GaN substrates, in turn providing motivation for bulk crystal growth methods able to produce large equi-axed crystal boules which can be cut into substrates with a variety of orientations. Our particular interest in this review is the prospect for semi-/non-polar III-nitride growth enabling LEDs with intrinsically high modulation speeds, although much work in the field has been motivated directly by development of more efficient devices emitting 
in the green and yellow spectral regions. LEDs fabricated in non-(0001) orientations also provide partial linear polarisation of the emitted light, which potentially offers novel degrees of freedom for data encoding in VLC.

a)

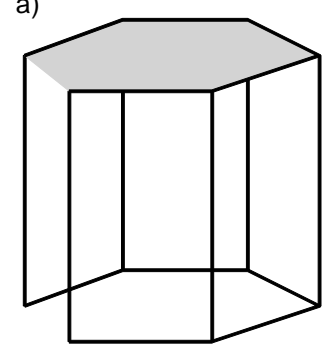

d)

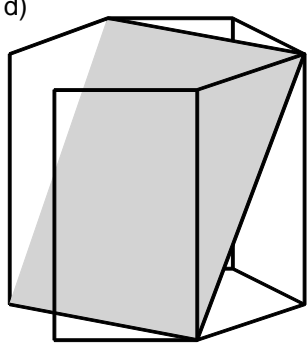

b)

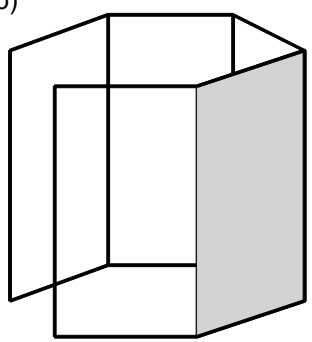

e)

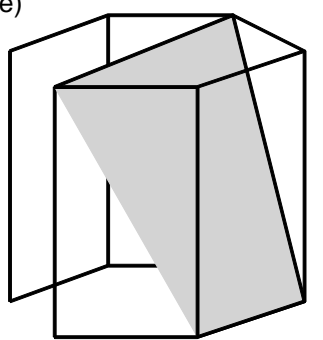

c)

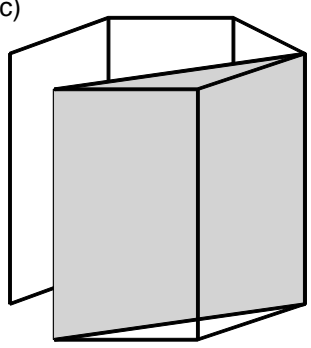

f)

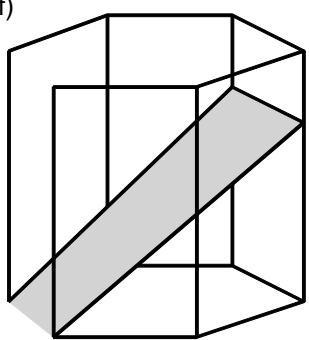

Figure 5: Important growth orientations in hexagonal GaN: a) $c$-plane (polar), b) $m$-plane (non-polar), c) $a$-plane (non-polar); d)-f) examples of semi-polar planes, d) $11 \overline{2} 2$, e) $10 \overline{1} 1$, f) $10 \overline{1} 3$. The plane shaded in grey represents the GaN crystal plane parallel to the substrate surface. It is shown in relation to a hexagonal prism larger than the conventional unit cell of $\mathrm{GaN}$, and the crystallographic $c$-axis is vertical in each case.

The wurtzite crystal structure lacks a centre of symmetry, and therefore III-nitride materials possess a spontaneous polarisation, whose magnitude depends on the material composition. This spontaneous polarisation makes an intrinsic contribution to the electric fields. In any kind of strained heterostructure, an additional field component arises owing to piezoelectric effects. InGaN-based QWs are under compressive strain of at least a few percent in typical LED structures, and in these circumstances the spontaneous and piezoelectric field contributions are of comparable magnitude, and generate net fields in the $\mathrm{MV} / \mathrm{cm}$ range. It should be emphasised that these internal electric fields are present even without an externally applied bias and have profound effects on the properties of III-nitride quantum wells [94]. The electric field within the quantum wells leads to a separation and reduced overlap of the electron and hole wavefunctions, a phenomenon referred to as the "quantum confined Stark effect" (QCSE) [94]. The QCSE, illustrated in Figure 5 leads to a redshift of the emission wavelength and, notably, to reduced radiative and Auger recombination rates [95], [96]. This means that the bandwidth of LEDs with a strong QCSE may be limited by the reduced recombination rates.

Generally, the QCSE in GaN-based QWs is strongest if grown on the polar c-plane orientation, for which the net polarisation field is perpendicular to the plane of the QWs. For the non-polar $a$ and $m$-plane orientations, the polarisation field is in the plane of the QWs, and no QCSE is present. Semi-polar planes, as illustrated by three examples of practical importance in Figure 5, present an intermediate case in which the QCSE is reduced in impact by the inclination of the polarisation direction to the plane of the QWs.
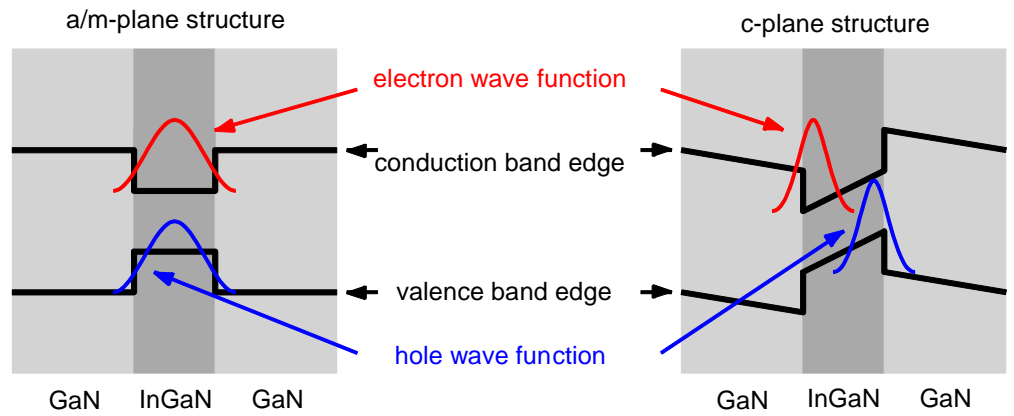

Figure 6: Illustration of the QCSE, where the vertical axis represents potential energy and the horizontal axis distance in the growth direction (the typical QW thickness is 2-3 nm). In $c$-plane GaN LEDs, the net electric field leads to a separation of the electron and hole wavefunctions and thus reduced carrier recombination rates. 
Most experimental evidence about the effect of growth orientation on carrier lifetimes is so far is based on photoluminescence lifetime (PL) measurements. Various groups reported a reduction of PL lifetime by up to two orders of magnitude when comparing semi/non-polar structures to $c$-plane structures [87]-[89], [92]. The first such report was by Waltereit et al. [97] who observed a PL lifetime reduction from 5.8 ns on a $c$-plane structure to $450 \mathrm{ps}$ on an $m$-plane structure. PL lifetimes between $100 \mathrm{ps}$ and $500 \mathrm{ps}$ have been reported by several groups and on a range of semi/non-polar planes [97]-[100]. If these short carrier lifetimes can be harnessed, they would readily provide modulation bandwidths on the order of $1 \mathrm{GHz}$ for VLC applications. High-speed electrically driven semi-/non-polar LEDs have not been reported yet, however, with the notable exception of the nanowire devices discussed below in section 3.2(e).

GaN can also be grown with a cubic (zinc-blende) crystal structure [101], [102] which does not have the drawback of a spontaneous polarisation. Piezoelectric fields may still be present in strained QWs, but the QCSE is generally of much less impact than in wurtzite-based device structures. However, high crystal quality is difficult to achieve, and growth typically relies on slow and cost-intensive molecular beam epitaxy [102], [103], thus inhibiting widespread use. Chichibu et al. [104] showed by time-resolved PL measurements that the carrier lifetime in cubic InGaN/GaN QWs is on the order of $200 \mathrm{ps}$, similar to that in comparable semi-/non-polar QWs. There are few reports of LEDs based on cubic GaN following the early work in ref. [78]. Recently Stark et al. reported green-emitting LEDs grown on microstructured Si substrates, using MOCVD, in a process which may be more viable for production than previous approaches [105]. To our knowledge, no high-speed electrically driven devices have yet been reported.

\section{c) Bespoke planar GaN epitaxy on $c$-plane sapphire substrates}

Section 3.2(b) discussed approaches to reduce the QCSE, and thus its effect on the bandwidth, by manipulating the crystal structure and growth direction. It is also possible to increase the carrier recombination rate in $c$-plane GaN LEDs by engineering the epitaxial layers in otherwise conventional structures. This can be achieved by doping of certain layers, varying the $\mathrm{InN}$ fraction in, and thickness of, the QWs, and introducing additional functional layers.

Shi et al. [106], [107] used n-type doped barrier layers in the LED QW region in order to improve the material quality and the radiative recombination rate. They attributed benefits to strain relaxation due to microstructure alteration upon silicon doping. Barrier doping also leads to a reduction of the QCSE through field screening, causing a blue-shift compared to an otherwise identical un-doped control LED sample. An improved modulation speed of $120 \mathrm{MHz}$ was achieved, under the same bias current, for the partially n-doped LED, compared to $40 \mathrm{MHz}$ from the un-doped control sample. The maximum output power was also enhanced by approximately 3 times [107].

$\mathrm{Li}$ and co-authors [108] used a shallow InGaN/GaN QW with low InN fraction to reduce the electrostatic field within the LED active area. An improvement in the output power of around 25\% was observed for the shallow QW device compared to a reference LED. This was attributed to an enhancement of the radiative recombination rate due to the reduced QCSE.

Lee et al. [109] used a low AlN-fraction AlGaN interlayer in between the active MQWs and the AlGaN electron-blocking layer, yielding an increase in output power by $29 \%$ compared to a control device. This was attributed to a higher hole concentration and improved hole distribution and, therefore, an enhanced radiative recombination rate, which also implies a higher bandwidth.

A recent report on cyan-emitting LEDs optimised for data communications achieved 3-dB E-O bandwidths of almost $1 \mathrm{GHz}$ under room-temperature operation, and corresponding output powers of $1.7 \mathrm{~mW}$ [110]. These results were achieved from devices with 4-period InGaN/GaN QW active regions, in which the GaN barrier layers had no intentional doping, and were reduced in thickness to an unusually small value of 5 $\mathrm{nm}$. Comparisons were made with a more conventional reference structure featuring 17-nm barriers, involving both experimental measurements on devices, and simulations. These indicated that modification of the active region could increase bandwidth twofold, and simulations confirmed a much more uniform distribution of radiative recombination throughout the active region in the case of the thin-well structure. 
A more radical approach to novel device structures than discussed in the previous section involves use of 3-dimensional so-called nanowire arrays ${ }^{1}$. Several forms of GaN nanowire LED have been demonstrated or proposed. However, the type most relevant to the present discussion are fabricated from arrays of nanowires epitaxially grown with their long axes perpendicular to a substrate, and processed so that many nanowires are interconnected in parallel over a macroscopic area in a finished device. Typically GaN nanowires are grown in the wurtzite phase, with a [0001] growth direction, and the crystallographic facets of each nanowire give it hexagonal prismatic shape. The conventional MOCVD techniques for doping and heterostructure growth can be adapted to nanowire growth, and allow growth of core-shell device structures [111]. In the situation as illustrated in Figure 7, InGaN-based QWs can be grown on the sidewalls of hexagonal prismatic nanowires in the non-polar $m$-plane orientation, whose benefits have already been discussed. Current efforts in GaN-based nanowire-LEDs are primarily driven by the prospect of developing highly efficient emitters in the green/yellow spectral range, for which the large recombination volume per unit area of substrate is a critical consideration [112]. However, pioneering results by Koester et al. [113] show that this technology also enables LEDs with GHz bandwidth.

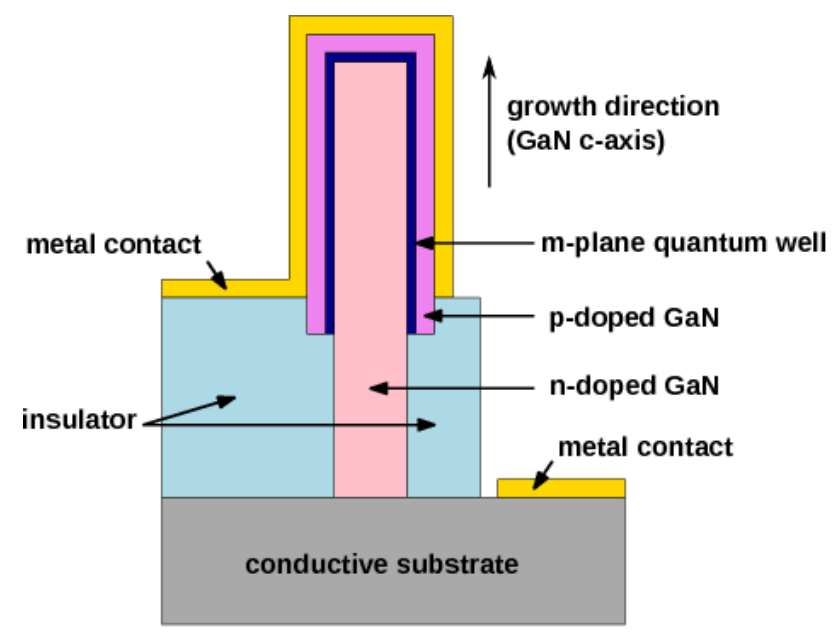

Figure 7: Schematic cross-section of an individual GaN nanowire element in a high-speed GaN nanowire LED. This embodiment assumes a conductive substrate, such as the Si used in the work of ref. [113].

The nanowire LEDs reported by Koester et al. featured nanowires of $15 \mu \mathrm{m}$ height, diameters ranging from $400-1500 \mathrm{~nm}$, and an areal density of $\sim 110$ per $(100 \mu \mathrm{m})^{2}$. In the large-area LEDs fabricated, typically 50 individual nanowires were estimated to contribute to light emission, owing to immature processing and contacting techniques. Figure 8 shows the optical output from these devices with a modulation at $1.1 \mathrm{GHz}$ and $1.3 \mathrm{GHz}$, respectively. From this result, it can be seen that the electrical to optical modulation bandwidth of the LED is between $1.1 \mathrm{GHz}$ and $1.3 \mathrm{GHz}$. To our knowledge, this is the highest bandwidth reported for a violet/blue emitting LED to date. Koester et al. [113] attribute this high bandwidth to the QCSE-free $m$-plane quantum wells in their device which reduce the carrier lifetime to a few $100 \mathrm{ps}$.

Currently, nanowire LEDs are still very challenging to fabricate and have modest optical output powers. However, the above results clearly show that advances in the field can lead to a new generation of high-speed LEDs for communications, particularly if improvements in growth and processing of nanowire arrays are pioneered for general lighting applications, and can be then applied to specialised data communications devices.

\footnotetext{
${ }^{1}$ The terms 'nanowire', 'nanocolumn' and 'nanorod' are used somewhat interchangeably in the literature. The nano-objects of interest here are typically $>100 \mathrm{~nm}$ in diameter, such that lateral quantum confinement effects are unimportant.
} 


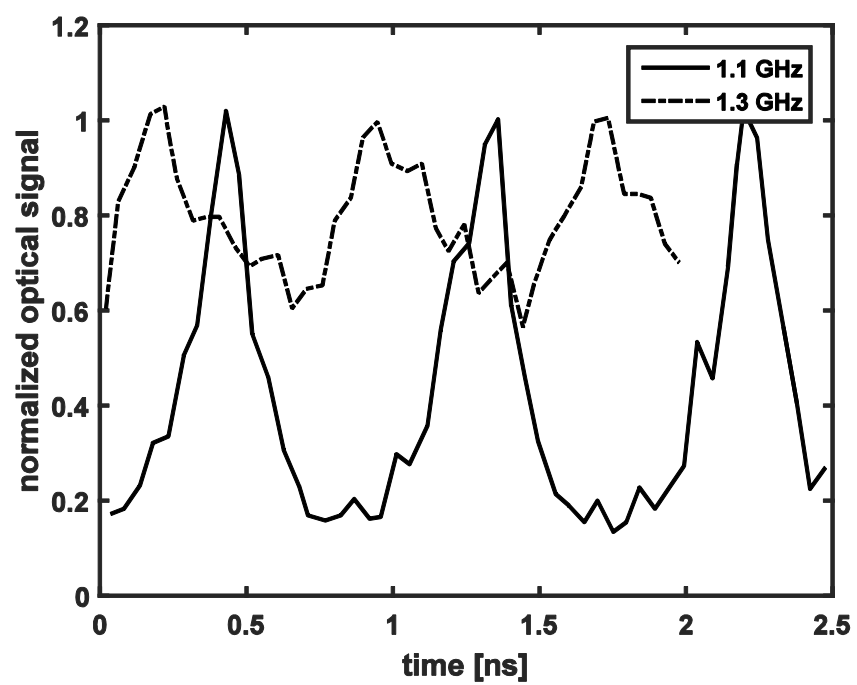

Figure 8: Modulated optical signal from a nanowire LED indicating a bandwidth of about 1.1 GHz. Data was taken from Koester et al. [113] and is normalized to the peak value of the optical pulses.

\section{e) High bandwidth GaN-based $\mu$ LEDs using conventional epistructures}

This section surveys work in which LEDs engineered to have small-area current injection regions have been developed for data communications using conventional planar epitaxial structures. Factors ultimately controlling modulation bandwidth are also discussed with reference to a conventional model for recombination dynamics. This approach has the advantage that existing mass-market epitaxial material is readily available for rapid implementation and commercialization.

In 2010, McKendry et al. reported the electrical-to-optical (E-O) modulation bandwidths measured from single pixels of GaN-based $\mu$ LED arrays having peak emission wavelengths of 370, 405 and $450 \mathrm{~nm}$ and pixel diameters of $72 \mu \mathrm{m}$ [23]. The highest bandwidth reported was $245 \mathrm{MHz}$, in the case of the $450 \mathrm{~nm}$-emitting $\mu \mathrm{LED}$. Although these $\mu$ LEDs were fabricated from standard commercially-available 2" c-plane sapphire-GaN LED epitaxial wafers, and were processed using photolithography and etching techniques, the bandwidths reported were an order of magnitude higher than typical off-the-shelf conventional chip GaN LEDs.

Following this work, McKendry et al. reported E-O modulation bandwidths measured from GaN $\mu$ LED arrays which consisted of $8 \times 8$ individually addressable pixels with diameters ranging from 14 to $84 \mu \mathrm{m}$, allowing the influence of the $\mu$ LED size on the bandwidth to be studied [114]. It was found that, generally speaking, the smaller $\mu$ LEDs demonstrated higher maximum modulation bandwidths than their larger area counterparts on the same array, with bandwidths $>400 \mathrm{MHz}$ reported for blue and green-emitting $\mu$ LEDs, the highest reported for GaN LEDs at that time. When the modulation bandwidth versus current density (that is, the injected current normalised to the active area of the $\mu$ LED) was examined for $\mu$ LEDs of different area, but from the same array, a clear relationship between bandwidth and current density was observed. Although the smaller $\mu$ LEDs showed a higher maximum bandwidth, each $\mu$ LED had a similar bandwidth for a given injected current density, as shown in Figure 9. 


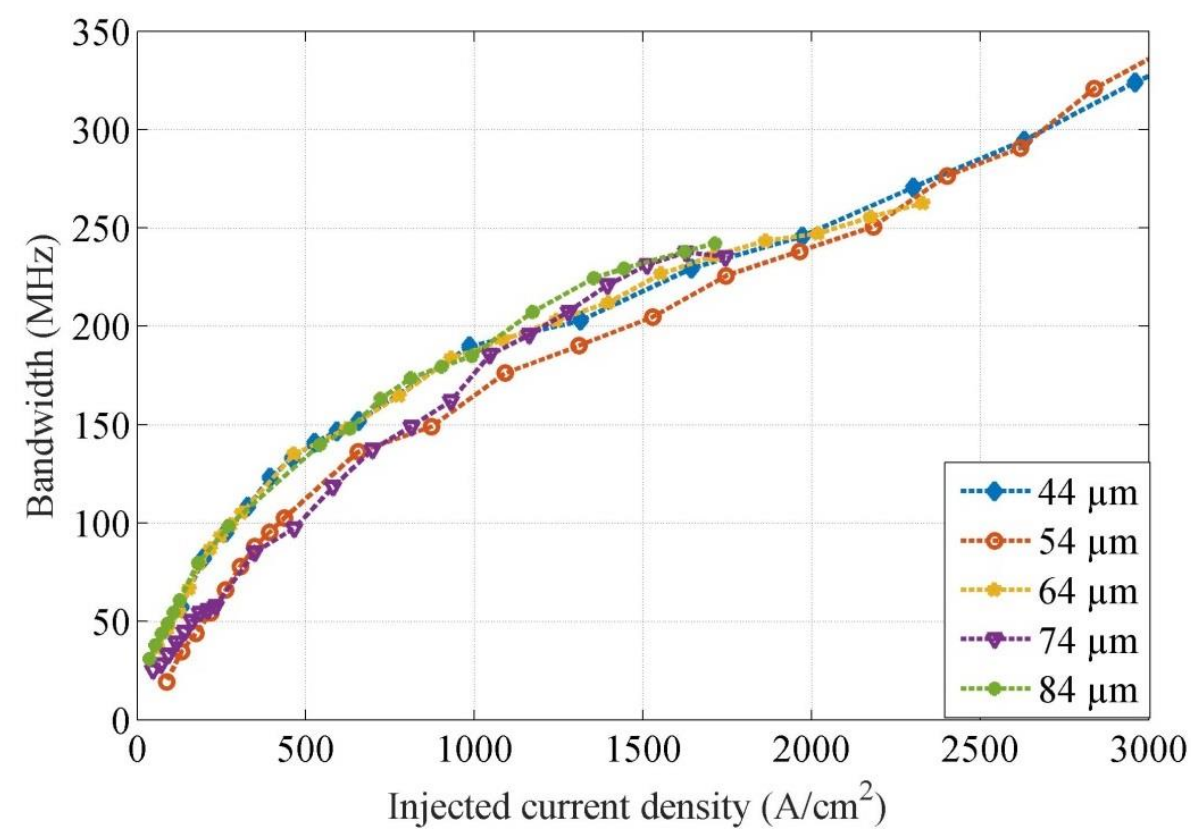

Figure 9: Electrical-to-optical bandwidth versus current density for $\mu$ LEDs of different diameters, but from the same $450 \mathrm{~nm}$-emitting $8 \times 8$ array. Experimental data reused from [114].

Since capacitance scales with device area this observation indicated that the modulation bandwidth of these $\mu$ LEDs was not limited by their $R C$ time constant, as is typically the case for conventional LEDs, otherwise one would expect to observe that for a given injected current density that the larger $\mu$ LEDs bandwidth would be lower than the bandwidth of the smaller $\mu$ LEDs. This observation that bandwidth varies with current density is best explained in terms of the modulation bandwidth of these $\mu$ LEDs being determined by the differential lifetime of the carriers within their quantum wells $\left(\tau_{\text {diff }}\right)$. The relationship between $\tau_{\text {diff }}$ and the carrier density has been extensively studied by others seeking to understand the 'efficiency droop' phenomenon that affects GaN LEDs, where the LED internal quantum efficiency (IQE) decreases as the injected current density increases. The simple ' $A B C$ ' model, used to describe carrier lifetimes in semiconductors [115], considers that the relationship between $\tau_{\text {diff }}$ and the carrier density $(N)$ is shown by equation (2):

$$
\tau(N)=\frac{1}{A+2 B N+3 C N^{2}}
$$

Here $A$ and $B$ are the Shockley-Read-Hall (SRH) non-radiative recombination and radiative recombination coefficients, respectively. $C$ is a higher-order non-radiative recombination coefficient, attributed to Auger recombination in other semiconductor materials, although in GaN-based LEDs this remains an ongoing topic of discussion [82].

From (2) it can be seen that as $N$ increases, $\tau_{\text {diff }}$ decreases. This provides an explanation for the behaviour observed in Figure 9 and for the high bandwidths shown by $\mu$ LEDs in general. Assuming a similar $N$ for similar current densities, each of the $\mu$ LEDs in Figure 4 would, therefore, be expected to exhibit a similar modulation response according to (2). Furthermore, as smaller area $\mu$ LEDs are able to be driven to higher current densities, this can explain how they generally exhibit higher modulation bandwidths than their larger area counterparts the capability to sustain higher carrier densities allows them to attain higher modulation bandwidth via a corresponding reduction in $\tau_{\text {diff. }}$.

High modulation bandwidths from micro-scale LEDs have been reported by several other research groups. Though there are variations in the structure and fabrication of these devices, high current density operation underpins the high bandwidths in each case. Liao et al., reported a blue-emitting $\mu$ LED with a gallium-doped zinc oxide current-confining aperture [116]. The current-confinement allowed modulation bandwidth of $225 \mathrm{MHz}$ to be demonstrated. Subsequently, Liao et al. reported current confinement in a greenemitting $\mu$ LED by the use of a ring-shaped electrode, with a bandwidth of $\sim 463 \mathrm{MHz}$ reported [27]. Maaskant $e t$ al. reported a 14-element $\mu$ LED array with a modulation bandwidth $>450 \mathrm{MHz}$ at a current of $70 \mathrm{~mA}(10$ $\mathrm{kA} / \mathrm{cm}^{2}$ ). Inter-connecting 14 elements allowed the output power to be increased, and a data rate of $500 \mathrm{Mb} / \mathrm{s}$ was subsequently demonstrated over a few $\mathrm{cm}$ of free space [117]. A cyan $\mu$ LED with a peak emission of 500 $\mathrm{nm}$ and a bandwidth of approximately $400 \mathrm{MHz}$ was reported by Wun et al. [118]. This emission of this $50 \mu \mathrm{m}$ 
diameter $\mu \mathrm{LED}$ was optimised for data transmission over polymer optical fibre (POF), and $1.07 \mathrm{Gbit} / \mathrm{s}$ data transmission was demonstrated over $50 \mathrm{~m}$ of POF.

These reports demonstrate that very high modulation bandwidths, compared to conventional chip LEDs, are possible using standard LED epitaxial material and processing techniques, by patterning the LED active area into $\mu$ LED structures. High-speed data transmission over free-space and guided wave that has been achieved with such high bandwidth $\mu$ LEDs will be discussed in subsequent sections.

\section{f) Plasmonic-enhanced LEDs}

A distinct approach to LEDs with high modulation bandwidths exploits resonant interactions between the QW excitations and surface plasmon polaritons, supported by proximal films or nanostructures of a suitable metal. Early demonstrations of such effects in III-nitride semiconductors were reported by Okamoto et al., who emphasized the resonant coupling condition possible between blue-emitting InGaN/GaN QWs and bulk-like silver [119]. The plasmonic coupling concept has now been applied to III-nitride LEDs quite widely [120], and recent work has specifically emphasised the potential for VLC. The commonest experimental methodology is to compare the performance of devices containing metal nanostructures with control devices without these. Zhu et al. reported on GaN-based plasmonic LEDs which had active areas of $8800 \mu^{2}$, but in which the epitaxial wafer was processed into nano-columns down the level of the QW active region, and then re-planarized by use of a spin-on glass [121]. LEDs in which discrete silver nanoparticles were embedded close to the QWs exhibited E-O bandwidths of $\sim 30 \mathrm{MHz}$, approximately twice those of control devices without the silver nanoparticles present. A different fabrication approach was followed by Yang et al., who exploited the so-called V-pits, of inverted hexagonal pyramid shape which develop naturally in certain MOCVD growth regimes [122]. Coating of a heavily V-pitted device surface with silver created a nano-structured metal layer, whose morphology was shown to be favourable for plasmonic coupling at relatively large separations of $>100 \mathrm{~nm}$ from the QW active region. A large plasmonic enhancement in E-O bandwidth was demonstrated for LEDs of $100 \mu \mathrm{m}$ diameter. At the highest dc drive voltage of $10 \mathrm{~V}$, a plasmonic-enhanced device had a bandwidth of $200 \mathrm{MHz}$, versus 115 $\mathrm{MHz}$ observed from a counterpart device without the silver layer. Full realization of the potential of plasmonic effects in high-speed III-nitride LEDs poses various challenges. For example, optimized metal nanostructures can be designed by finite difference time domain electromagnetic modelling, but can only be approximated by current fabrication technologies, while practical devices require additional continuous metal layers to spread current and allow external contacting.

\section{High-speed data communication}

Optical wireless communication (OWC) for indoor wireless local area networks (WLAN) was first studied at IBM Laboratory by Gfeller and Bapst in 1979 using infrared radiation [123]. With the development and wide availability of GaN LEDs in the visible range and due to advantages outlined in section 2, VLC systems have, however, emerged as the key wireless technology that has the potential to overcome the looming RF 'spectrum crunch' [124]. This section surveys high-speed VLC demonstrations, with a focus on techniques adopted to improve the data communication capacity of a VLC link.

Table II: Survey of practical demonstrations of VLC systems

\begin{tabular}{|c|c|c|c|c|c|c|c|}
\hline Year & Sources & $\begin{array}{l}\text { Modulation } \\
\text { scheme }\end{array}$ & Multiplexing & $\begin{array}{c}\text { Pre- } \\
\text { equalisation }\end{array}$ & $\begin{array}{c}\text { Post- } \\
\text { equalisation }\end{array}$ & Data rate (BER threshold) & Ref. \\
\hline 2016 & RGB LEDs & OFDM & WDM & & & $10 \mathrm{Gbit} / \mathrm{s}\left(\mathrm{BER}<3.8 \times 10^{-3}\right)$ & {$[18]$} \\
\hline 2016 & $\mu \mathrm{LED}$ & PAM & Spatial & & $\mathrm{X}$ & $7 \mathrm{Gbit} / \mathrm{s}\left(\mathrm{BER}<3.8 \times 10^{-3}\right)$ & [33] \\
\hline 2016 & $\mu \mathrm{LED}$ & OFDM & & & & $5 \mathrm{Gbit} / \mathrm{s}\left(\mathrm{BER}<3.8 \times 10^{-3}\right)$ & {$[24]$} \\
\hline 2016 & $\mu \mathrm{LED}$ & PAM & & & $\mathrm{X}$ & $3.5 \mathrm{Gbit} / \mathrm{s}\left(\mathrm{BER}<3.8 \times 10^{-3}\right)$ & {$[24]$} \\
\hline 2016 & $\mu \mathrm{LED}$ & PAM & & $\mathrm{X}$ & & 2 Gbit/s $\left(\right.$ BER $\left.<10^{-12}\right)$ & [125] \\
\hline 2016 & Laser & OOK & & & & $1 \mathrm{Gbit} / \mathrm{s}$ & [126] \\
\hline 2015 & RGB laser & OFDM & WDM & & & 12.4Gbit/s $\left(\mathrm{BER}<3.6 \times 10^{-3}\right)$ & [127] \\
\hline 2015 & RGB laser & OFDM & WDM & & & $14 \mathrm{Gbit} / \mathrm{s}\left(\mathrm{BER}<3.6 \times 10^{-3}\right)$ & [31] \\
\hline 2015 & Blue Laser & OFDM & & & & 9 Gbi t/s $\left(\mathrm{BER}<3.6 \times 10^{-3}\right)$ & [128] \\
\hline 2015 & $\begin{array}{c}\text { Blue laser }+ \\
\text { phosphor }\end{array}$ & OFDM & & & & $5 \mathrm{Gbit} / \mathrm{s}\left(\mathrm{BER}<3.8 \times 10^{-3}\right)$ & {$[43]$} \\
\hline 2015 & $\begin{array}{c}\text { Blue laser }+ \\
\text { phosphor }\end{array}$ & OFDM & Spatial & & & $10 \mathrm{Gbit} / \mathrm{s}\left(\mathrm{BER}<3.8 \times 10^{-3}\right)$ & {$[42]$} \\
\hline 2015 & RYGB & CAP & WDM & $\mathrm{X}$ & $\mathrm{X}$ & $8 \mathrm{Gbit} / \mathrm{s}\left(\mathrm{BER}<3.8 \times 10^{-3}\right)$ & [34] \\
\hline 2015 & RYGB & OFDM & WDM & & & $5.6 \mathrm{Gbit} / \mathrm{s}\left(\mathrm{BER}<3.8 \times 10^{-3}\right)$ & [30], \\
\hline
\end{tabular}




\begin{tabular}{|c|c|c|c|c|c|c|c|}
\hline & & & & & & & [129] \\
\hline 2015 & RGB & CAP & WDM & $\mathrm{X}$ & & $4.5 \mathrm{Gbit} / \mathrm{s}\left(\mathrm{BER}<3.8 \times 10^{-3}\right)$ & {$[130]$} \\
\hline 2015 & $\begin{array}{l}\text { Blue laser + } \\
\text { phosphor }\end{array}$ & OFDM & & & & $4 \mathrm{Gbit} / \mathrm{s}\left(\mathrm{BER}<9.7 \times 10^{-5}\right)$ & [131] \\
\hline 2015 & $\begin{array}{l}\text { Blue laser + } \\
\text { phosphor }\end{array}$ & $\mathrm{OOK}$ & & & & $4 \mathrm{Gbit} / \mathrm{s}\left(\mathrm{BER}<3.8 \times 10^{-3}\right)$ & {$[132]$} \\
\hline 2015 & $\begin{array}{l}\mu \mathrm{LED}+\text { polymer } \\
\text { colour converter }\end{array}$ & OFDM & WDM & & & $2.3 \mathrm{Gbit} / \mathrm{s}\left(\mathrm{BER}<3.8 \times 10^{-3}\right)$ & {$[38]$} \\
\hline 2015 & White LED & $\begin{array}{l}\text { OFDM (bit and } \\
\text { power loading) }\end{array}$ & & $\mathrm{X}$ & & $2 \mathrm{Gbit} / \mathrm{s}\left(\mathrm{BER}<3.8 \times 10^{-3}\right)$ & [133] \\
\hline 2015 & White LED & OFDM & & & & 1.6 Gbit/s $\left(\mathrm{BER}<3.8 \times 10^{-3}\right)$ & [134] \\
\hline 2015 & White LED & QAM-OFDM & & $\mathrm{X}$ & & $1.4 \mathrm{Gbit} / \mathrm{s}\left(\mathrm{BER}<3.8 \times 10^{-3}\right)$ & $\begin{array}{l}{[135],} \\
{[136]}\end{array}$ \\
\hline 2015 & White LED & PAM & & & $\mathrm{X}$ & $1.1 \mathrm{Gbit} / \mathrm{s}$ & [137] \\
\hline 2015 & White LED & QAM-OFDM & & $\mathrm{X}$ & & $750 \mathrm{Mbit} / \mathrm{s}\left(\mathrm{BER}<3.8 \times 10^{-3}\right)$ & [138] \\
\hline 2015 & White LED & QAM-OFDM & & $\mathrm{X}$ & & $682 \mathrm{Mbit} / \mathrm{s}\left(\mathrm{BER}<2.5 \times 10^{-3}\right)$ & [139] \\
\hline 2015 & LED & OFDM & Spatial & & & $1.4 \mathrm{Gbit} / \mathrm{s}\left(\mathrm{BER}<3.8 \times 10^{-3}\right)$ & [140] \\
\hline 2015 & White LED & OFDM & Spatial & & & $1.3 \mathrm{Gbit} / \mathrm{s}\left(\mathrm{BER}<3.8 \times 10^{-3}\right)$ & [141] \\
\hline 2015 & Nanowire & $\mathrm{OOK}$ & & & & $1 \mathrm{Gbit} / \mathrm{s}$ & {$[113]$} \\
\hline 2014 & & 16-QAM & Polarisation & & $\mathrm{X}$ & $1 \mathrm{Gbit} / \mathrm{s}\left(\mathrm{BER}<3.8 \times 10^{-3}\right)$ & [142] \\
\hline 2014 & Blue LED & 4-QAM & Spatial & & $\mathrm{X}$ & $500 \mathrm{Mbit} / \mathrm{s}\left(\mathrm{BER}<3.8 \times 10^{-3}\right)$ & [143] \\
\hline 2014 & White LED & OOK-NRZ & Spatial & $\mathrm{X}$ & & $50 \mathrm{Mbit} / \mathrm{s}\left(\mathrm{BER}<3.8 \times 10^{-3}\right)$ & [144] \\
\hline 2014 & RGB & QAM & Wavelength & & & $\begin{array}{c}4.22-\text { Gbit/s }\left(\mathrm{BER}<3.8 \times 10^{-}\right. \\
3)\end{array}$ & {$[145]$} \\
\hline 2014 & White LED & OOK-NRZ & & & $\mathrm{X}$ & $340 \mathrm{Mbit} / \mathrm{s}\left(\mathrm{BER}<2 \times 10^{-3}\right)$ & [146] \\
\hline 2014 & White LED & NRZ-OOK & & $\mathrm{X}$ & $\mathrm{X}$ & $550 \mathrm{Mbit} / \mathrm{s}(\underset{9}{\mathrm{BER}})$ & [147] \\
\hline 2014 & $\mu \mathrm{LED}$ & $\begin{array}{l}\text { OFDM (bit and } \\
\text { power loading) }\end{array}$ & & & & $3 \mathrm{Gbit} / \mathrm{s}\left(\mathrm{BER}<3.8 \times 10^{-3}\right)$ & [25] \\
\hline 2014 & $\begin{array}{c}\mu \mathrm{LED}+\text { polymer } \\
\text { colour converter } \\
\text { (white) }\end{array}$ & OFDM & & & & $1.68 \mathrm{Gbit} / \mathrm{s}\left(\mathrm{BER}<3.8 \times 10^{-3}\right)$ & [26] \\
\hline 2013 & Blue Laser & OOK-NRZ & & & & $2.5 \mathrm{Gbit} / \mathrm{s}\left(\mathrm{BER}<3.8 \times 10^{-3}\right)$ & {$[148]$} \\
\hline 2013 & Red LED & OOK-NRZ & & & & $300 \mathrm{Mbit} / \mathrm{s}\left(\mathrm{BER}<10^{-9}\right)$ & [149] \\
\hline 2013 & White LED & $\begin{array}{l}\text { Rate adaptive } \\
\text { OFDM }\end{array}$ & & & & $500 \mathrm{Mbit} / \mathrm{s}\left(\mathrm{BER}<3.8 \times 10^{-3}\right)$ & {$[150]$} \\
\hline 2013 & White LED RGB & OOK-NRZ & Wavelength & $\mathrm{X}$ & & $477 \mathrm{Mbit} / \mathrm{s}\left(\mathrm{BER}<3.8 \times 10^{-3}\right)$ & [151] \\
\hline 2013 & White LED & OOK-NRZ & & & $\mathrm{X}$ & $170 \mathrm{Mbit} / \mathrm{s}\left(\mathrm{BER}<3.8 \times 10^{-3}\right)$ & $\begin{array}{l}{[152],} \\
{[153]}\end{array}$ \\
\hline 2013 & RGB & CAP & Wavelength & $\mathrm{X}$ & $\mathrm{X}$ & $3.22 \mathrm{Gbit} / \mathrm{s}\left(\mathrm{BER}<3 \times 10^{-3}\right)$ & $\begin{array}{l}{[154],} \\
{[155]}\end{array}$ \\
\hline 2013 & $\mu$ LED (blue) & OOK & Spatial & & & $1.5 \mathrm{Gbit} / \mathrm{s}\left(\mathrm{BER}<<1 \times 10^{-10}\right)$ & [156] \\
\hline 2013 & White LED & OFDM & Spatial & & & $1 \mathrm{Gbit} / \mathrm{s}\left(\mathrm{BER}<2 \times 10^{-3}\right)$ & {$[157]$} \\
\hline 2013 & RGB & QAM-OFDM & Spatial & & & $575 \mathrm{Mbit} / \mathrm{s}\left(\mathrm{BER}<2 \times 10^{-3}\right)$ & {$[158]$} \\
\hline 2012 & RGB(WDM) & OFDM & Spatial & & & $3.4 \mathrm{Gbit} / \mathrm{s}\left(\mathrm{BER}<3.8 \times 10^{-3}\right)$ & [159] \\
\hline 2012 & RGB & OFDM & & & & $2.1 \mathrm{Gbit} / \mathrm{s}\left(\mathrm{BER}<10^{-3}\right)$ & {$[160]$} \\
\hline 2012 & White LED & CAP & & $\mathrm{X}$ & $\mathrm{X}$ & $1.1 \mathrm{Gbit} / \mathrm{s}\left(\mathrm{BER}<10^{-3}\right)$ & {$[161]$} \\
\hline 2012 & White LED & $\begin{array}{l}\text { OFDM (bit and } \\
\text { power loading) }\end{array}$ & & & & $1 \mathrm{Gbit} / \mathrm{s}\left(\mathrm{BER}<3.8 \times 10^{-3}\right)$ & {$[162]$} \\
\hline 2012 & RGB & OOK & Wavelength & $\mathrm{X}$ & $\mathrm{X}$ & $614 \mathrm{Mbit} / \mathrm{s}$ & {$[163]$} \\
\hline 2012 & RGB & DMT-QAM & Wavelength & & & $1.25 \mathrm{Gbit} / \mathrm{s}\left(\mathrm{BER}<2 \times 10^{-3}\right)$ & [164] \\
\hline 2011 & RGB & DMT-QAM & Wavelength & & & $813 \mathrm{Mbit} / \mathrm{s}\left(\mathrm{BER}<2 \times 10^{-3}\right)$ & {$[165]$} \\
\hline 2010 & $\mu$ LED (blue) & $\mathrm{OOK}$ & & & & $1 \mathrm{Gbit} / \mathrm{s}\left(\mathrm{BER}<1 \times 10^{-10}\right)$ & [23] \\
\hline 2010 & White LEDs & DMT-QAM & & & & $513 \mathrm{Mbit} / \mathrm{s}\left(\mathrm{BER}<2 \times 10^{-3}\right)$ & {$[166]$} \\
\hline 2010 & White LEDs & OOK-NRZ & & & & $230 \mathrm{Mbit} / \mathrm{s}\left(\mathrm{BER}<2 \times 10^{-3}\right)$ & {$[167]$} \\
\hline 2009 & White LEDs & DMT-QAM & & & & $200+\mathrm{Mbit} / \mathrm{s}\left(\mathrm{BER}<10^{-3}\right)$ & {$[168]$} \\
\hline 2009 & White LEDs & OOK-NRZ & & & & $125 \mathrm{Mbit} / \mathrm{s}$ & [169] \\
\hline 2009 & White LEDs & OOK-NRZ & & & $\mathrm{X}$ & $100 \mathrm{Mbit} / \mathrm{s}\left(\mathrm{BER}<10^{-9}\right)$ & [83] \\
\hline 2008 & White LEDs & OOK-NRZ & & $\mathrm{X}$ & & $80 \mathrm{Mbit} / \mathrm{s}\left(\mathrm{BER}<10^{-6}\right)$ & {$[22]$} \\
\hline 2008 & White LEDs & OOK-NRZ & & $\mathrm{X}$ & & $40 \mathrm{Mbit} / \mathrm{s}\left(\mathrm{BER}<10^{-6}\right)$ & [170] \\
\hline 2007 & White LEDs & DMT-QAM & & & & $100 \mathrm{Mbit} / \mathrm{s}\left(\mathrm{BER}<10^{-6}\right)$ & {$[171]$} \\
\hline 2006 & White LEDs & OFDM & & & & - & [172] \\
\hline 2002 & White LEDs & BPSK & & & & - & [173] \\
\hline
\end{tabular}




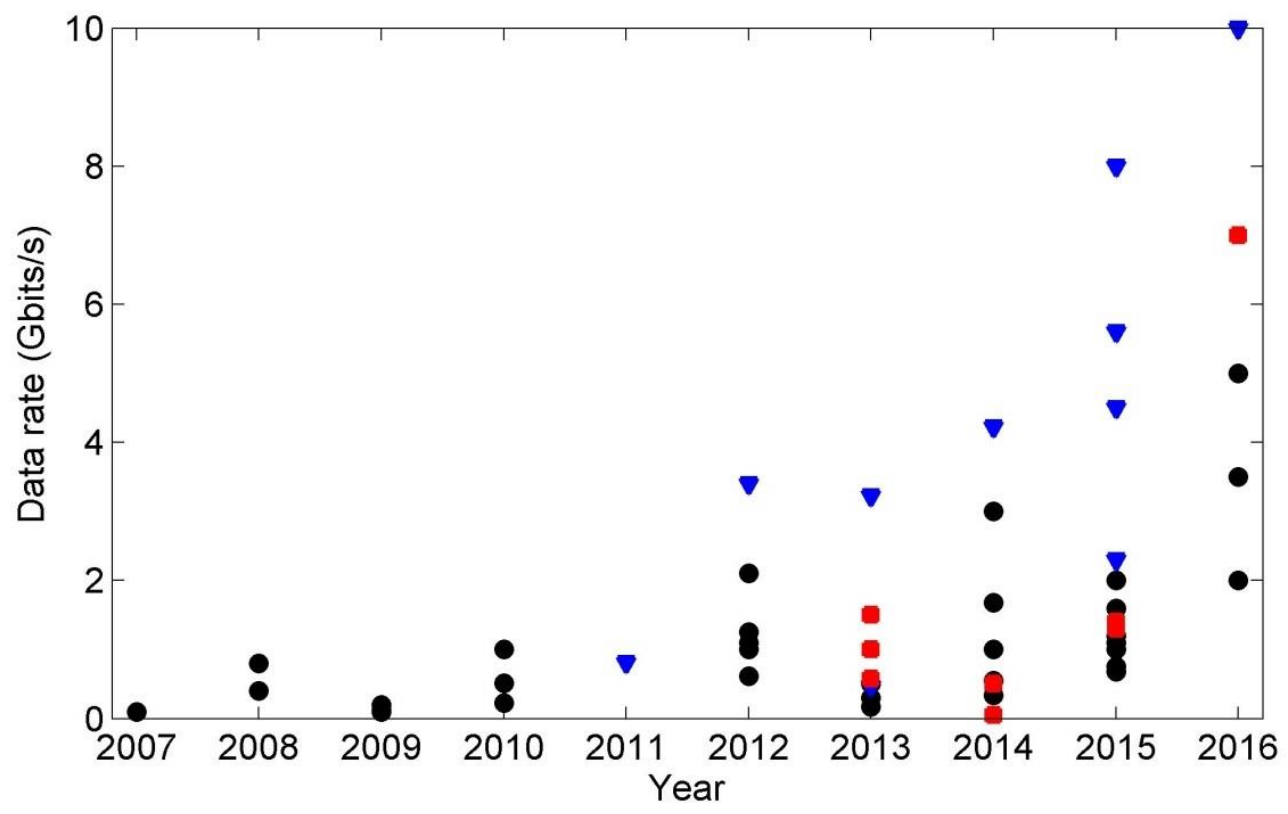

Figure 10: Progress in the reported data rates using visible GaN LEDs, black (circular) dots representing demonstration without any multiplexing, red (square) those with spatial multiplexing and blue (triangular) those with WDM.

Table II and Figure 10 clearly demonstrate that the research interest in VLC systems grew significantly over the last decade. There were few VLC demonstrations using white LEDs before 2008 and reported data rates were in the range of $100 \mathrm{Mbit} / \mathrm{s}$ using analogue equalisation or discrete Multitone (DMT) techniques [22], [170], [171]. By 2012, Gbit/s systems were demonstrated using a single white LED [161], [162]. More recently, researchers were able to achieve a $2 \mathrm{Gbit} / \mathrm{s}$ link using a commercial white LED [133]. The introduction of $\mu L E D s$ for VLC has further fuelled the improvement in the data rate. McKendry et al.showed a 1 Gbit/s free space VLC link using OOK without any equalisation [23]. Using the optimum bit and power loading DCOOFDM, the UP-VLC project demonstrated a 3 Gbit/s link 2013 [25] and more recently a 5 Gbit/s link [24] using a single blue $\mu \mathrm{LED}$.

Table I also clearly demonstrates the evolution of the following key technologies that enabled multiGbit/s data rates:

a) Improving the available bandwidth by adopting devices with a higher bandwidth

b) Efficient use of available bandwidth by adopting spectrally efficient multilevel modulations such as PAM or OFDM

c) Advanced signal processing such as pre-equalization, post-equalization and bit and power loading (for OFDM)

d) Exploiting the spatial dimension using spatial diversity and/or multiplexing

e) Exploiting the colour dimension using WDM

These key technologies that have enabled multi-Gbit/s VLC systems are discussed in the following sections.

\subsection{Modulation schemes}

The coherent transmission that has served well for RF communications is not cost-effective for VLC systems [48]. The low-cost VLC (and OWC in general) systems use optical intensity modulation (IM) and a direct detection (DD) receiver (referred to as IM/DD), i.e. information is modulated in the instantaneous intensity of an optical carrier, and the receiver detects the optical intensity of the incoming signal [48]. As a result, there are fundamental differences between RF and optical communications: a) information in OWC is carried on the intensity of an optical carrier, contrary to the amplitude and phase of the RF carrier, and the receiver detects the optical intensity of the incoming signal, b) optical signals are real-valued and unipolar whereas RF signals can be complex-valued and bipolar, and c) the signal-to-noise ratio (SNR) is proportional to the square of the received optical power, rather than the square of the carrier amplitude in the case of RF. As a result, only the baseband transmission is cost-effective for VLC systems i.e. there is no up-conversion to the carrier frequency using a local oscillator. Moreover, bipolar signalling schemes must be biased with a DC level 
to make them unipolar. Consequently, the modulation schemes employed in RF systems may need to be modified when applying them to VLC systems, which results in reduced spectral and power efficiencies [174].

Selecting a modulation technique depends on a number of key criteria. The important criteria that are often used as benchmarks for comparison of modulation schemes are listed below:

a. $\quad$ Power Efficiency: Power efficiency is the most important criterion when evaluating modulation techniques. The electrical power efficiency $\eta_{p}$ is the average electrical power required to achieve a given error probability at a given data rate and is defined as [48], [175]:

$$
\eta_{p}=\frac{E_{\text {pulse }}}{E_{b}}
$$

where $E_{\text {pulse }}$ is the energy per pulse and $E_{b}$ is the average energy per bit.

b. Bandwidth Efficiency: Bandwidth efficiency, also refer to as spectral efficiency, determines the number of bits that can be assigned per unit available bandwidth (i.e. bits/s/Hz) and is defined as:

$$
\eta_{B}=\frac{R_{b}}{B}
$$

where $R_{b}$ is the achievable bit rate and $B$ is the bandwidth of the system.

c. Receiver sensitivity: The receiver sensitivity is defined as the minimum optical power required to achieve a desirable error probability. The receiver sensitivity is related to power efficiency. The ratio of electrical power to optical power is dependent on the absolute level of optical power [176] and modulation schemes. Note that the smaller the receiver sensitivity, the better is the system, as such a system requires less power to achieve the desirable error probability.

The modulation schemes for VLC systems may be classified into a) baseband modulation and b) subcarrier modulation (SCM). The popular baseband modulation schemes include on-off keying (OOK), pulse amplitude modulation (PAM), pulse position modulation (PPM) and pulse interval modulation (PIM). The subcarrier base modulation includes orthogonal frequency division multiplexing (OFDM) and carrier-less amplitude and phase (CAP) modulation. There are other forms of modulation known as colour shift keying (CSK), which are based on constellation mapping by varying the colour space in the chromaticity diagram [19], [177]. However, the high-speed practical VLC demonstrations are geared towards the use of OOK, PAM, OFDM, and CAP. An overview of these modulation schemes is given in the following sub-sections.

\section{a) On-off keying (OOK)}

OOK is the simplest form of baseband modulation for IM/DD where information is sent by switching a source 'ON' and 'OFF' at a rapid speed. In the OOK non-return-to-zero (OOK-NRZ) scheme, 'ones' and 'zeros' are represented by the presence or absence, respectively, of an optical pulse for the bit duration, as illustrated in the time waveforms in Figure 11(a). The popularity of OOK is due to its simplicity in implementation and power efficiency. For example, a digital switching circuit like a NAND gate can serve as the driver for switching the light sources 'ON' and 'OFF' [45] and a simple digital comparator at the receiver can recover the binary data. Because of the simple driver circuit requirement, it is easy to fabricate integrated driver circuits that can drive a large number of LEDs independently. McKendry et al. demonstrated a Complimentary Metal-Oxide-Semiconductor (CMOS) based driver for GaN LED that can individually and independently control a 16×16 array of $\mu$ LEDs [23]. OOK-NRZ has been widely adopted for VLC, see Table 1 . Le Minh et al.adapted an OOK-NRZ scheme with pre/post equalisation and demonstrated up to $100 \mathrm{Mbit} / \mathrm{s}$ using commercial white LEDs [22], [83], [170]. Vuvic et al.demonstrated data rates up to $230 \mathrm{Mbits} / \mathrm{s}$ at 1000 lux [167]. Fujimoto et al. used a pre-emphasis circuit with OOK-NRZ to achieve a data rate of $477 \mathrm{Mbits} / \mathrm{s}$ using a red LED [151] and $614 \mathrm{Mbits} / \mathrm{s}$ by adopting analogue pre- and post-equalisation circuits [163]. Using a commercial phosphor-based white LEDs, Li et al.demonstrated $550 \mathrm{Mbit} / \mathrm{s}$ real-time VLC [147]. Using high bandwidth $\mu$ LEDs, up to 1.7 Gbits/s single-channel VLC links were recently demonstrated [23], [24], [156].

\section{b) Pulse Amplitude Modulation (PAM)}

In an $L$-PAM scheme (where typically $L=2^{\mathrm{M}}$ and $M$ is a positive integer), a pulse is selected from the alphabets of $\{0,1,2, \ldots,(L-1)\}$ to represent an $M$-bits input symbol. The spectral efficiency of multi-level PAM increases linearly with the bit resolution, but at the cost of reduced power efficiency [178], [179]. In addition to the optical power penalty due to multi-level signalling, multi-level PAM further suffers from optical power penalty due to the LED non-linearity. Hence, it was experimentally demonstrated that 4-PAM (Figure 
11(b)) offers improved performance in comparison to higher level PAM even in severely band-limited systems [125], [180].

Yang et al. [181] introduced differential PAM for bandlimited VLC systems. In the diff erential PAM scheme, signals with equal amplitudes but a unit time delay are transmitted through diff erent LEDs. Optical signals from these LEDs are superimposed in free space and detected using a single optical receiver. A 100 Mbits/s link was demonstrated with differential PAM using white LEDs. Li et al.demonstrated that it is feasible to generate multi-level PAM by linearly combining optical intensity in free space from spatially separated multiple LEDs [182]. This superposed PAM (SPAM) is insensitive to the non-linearity of the individual LEDs.

\section{c) Pulse position modulation (PPM)}

PPM (Figure 11(c)) is an orthogonal modulation scheme where information is carried in the position of a pulse within the symbol period. Each PPM symbol consists of $L$ time slots. Within the PPM symbol, all slots except one are empty and the temporal position of this pulse within the symbol period carries the information. Hence, PPM requires both slot and symbol synchronization at the receiver and is therefore vulnerable to loss of synchronization. However, PPM is well known for its power efficiency and has a long legacy of application in fibre optics and space communications [183], [184].

Most of the VLC systems have a high SNR, but limited bandwidth due to the use of phosphor-containing LEDs [185]. Because of the poor spectral efficiency of PPM, there is a limited adoption of PPM for high-speed VLC systems. Nonetheless, PPM is adopted in the IEEE 802.15.7 standard and the IrDA serial data communication standard [19]. IEEE 802.15.7 recommend a variable PPM, which changes the duty cycle to achieve a required dimming level [19]. There are also a number of variations to standard PPM including differential PPM (DPPM), overlapping PPM (OPPM) multiple PPM (MPPM), and expurgated PPM (EPPM). The study of these modulation schemes is not within the scope of this review. The interested reader can refer to references [186]-[189].

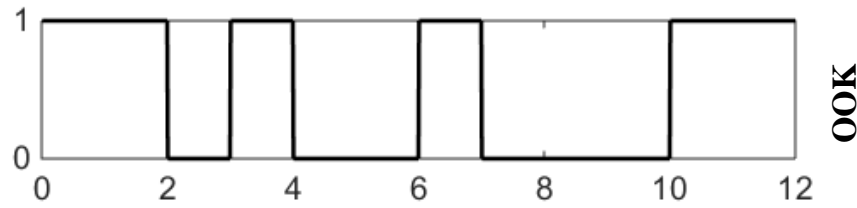

(a)
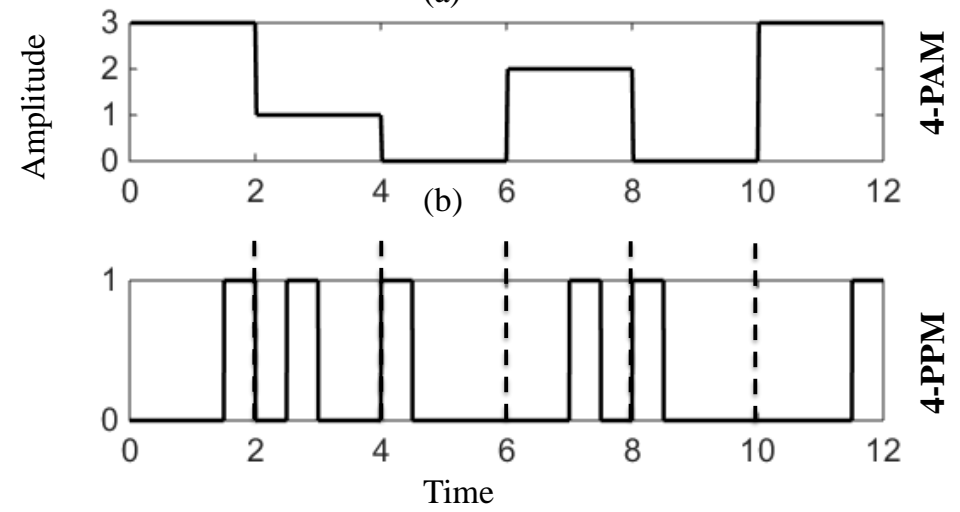

(c)

Figure 11: Time waveforms of (a) OOK, (b) 4-PAM, and (c) 4-PPM for a binary input sequence of $\{110100100011\}$ for the same data rate

\section{d) Carrier-less amplitude and phase modulation}

CAP bears a similarity to quadrature amplitude modulation (QAM), as both schemes use orthogonal bases to transmit two data streams in parallel. Unlike QAM, however, CAP does not use sinusoidal carriers to generate orthogonal components. Instead, CAP uses a pair of digital filters whose impulse response forms a Hilbert transform pair used as the orthogonal bases. Hence, the CAP is generated by combining two PAM signals which are convolved with these filters. If the two filters are chosen as sine and a cosine, then CAP offers identical performance to QAM [190]. The key advantages of CAP over QAM, however, is a simpler implementation 
[190] as it is not necessary to modulate the baseband CAP signal with quadrature carriers. This makes CAP an attractive scheme for IM/DD systems.

The impulse responses of the two orthogonal filters can be calculated using time-domain multiplication of cosine and sine with a root-raised cosine filter (RRCF) for in-phase (I) and quadrature phase (Q) channels, respectively. Figure 12 (a) and (b) show the transmitter pulse shaping filters for I and Q channels along with cosine and sine and root-raised cosine filter (RRCF) impulse response. Three parameters determine the impulse response of the transmitter filters: the sine and cosine frequency, the RRCF roll off factor $\alpha$ and the filter length (i.e. number of filter taps) [191]. The frequency can be arbitrarily chosen as long as it is higher than the highest frequency of the SRRC. The roll-off factor $\alpha$ determines the minimum bandwidth requirement and the filter length. The minimum passband bandwidth requirement for a CAP signal is $R_{s}(1+\alpha)$ where $R_{s}$ is the baud rate and $0 \leq \alpha \leq 1$. This means efficient use of available bandwidth can be achieved by choosing the value $\alpha$ close to zero. This, however, increases the complexity as a larger number of taps are required to meet the roll-off factor [191]. The smaller roll-off factor also increases the peak-to-average-power-ratio (PAPR) [192] which is potentially an issue for a limited dynamic range system.

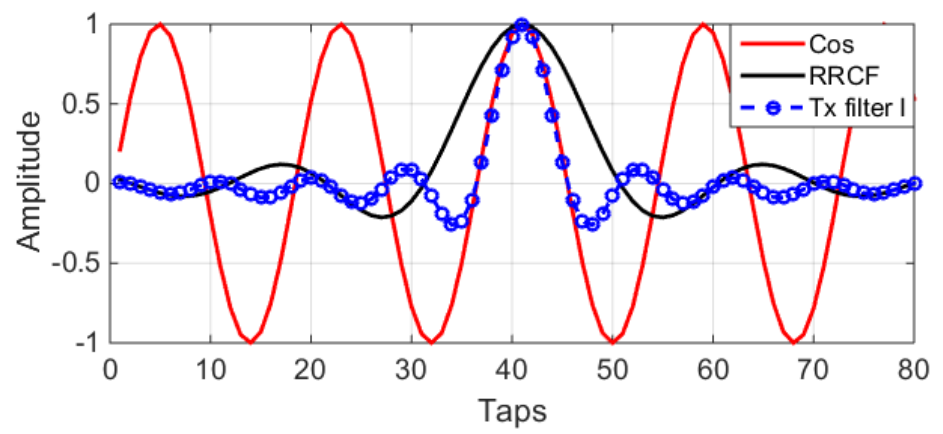

(a)

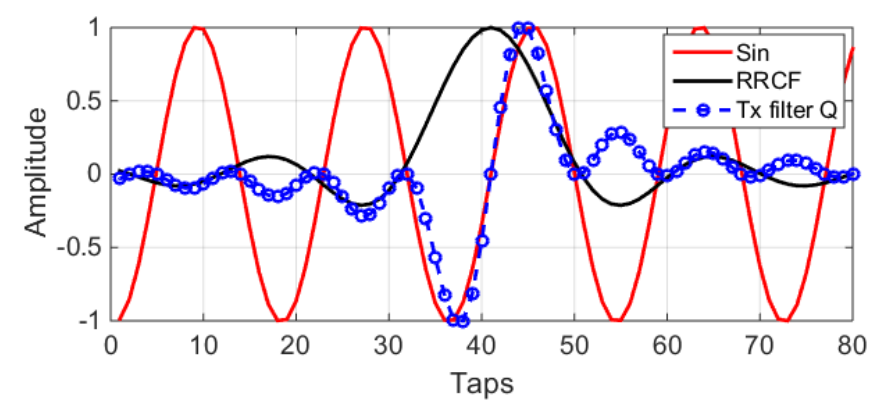

(b)

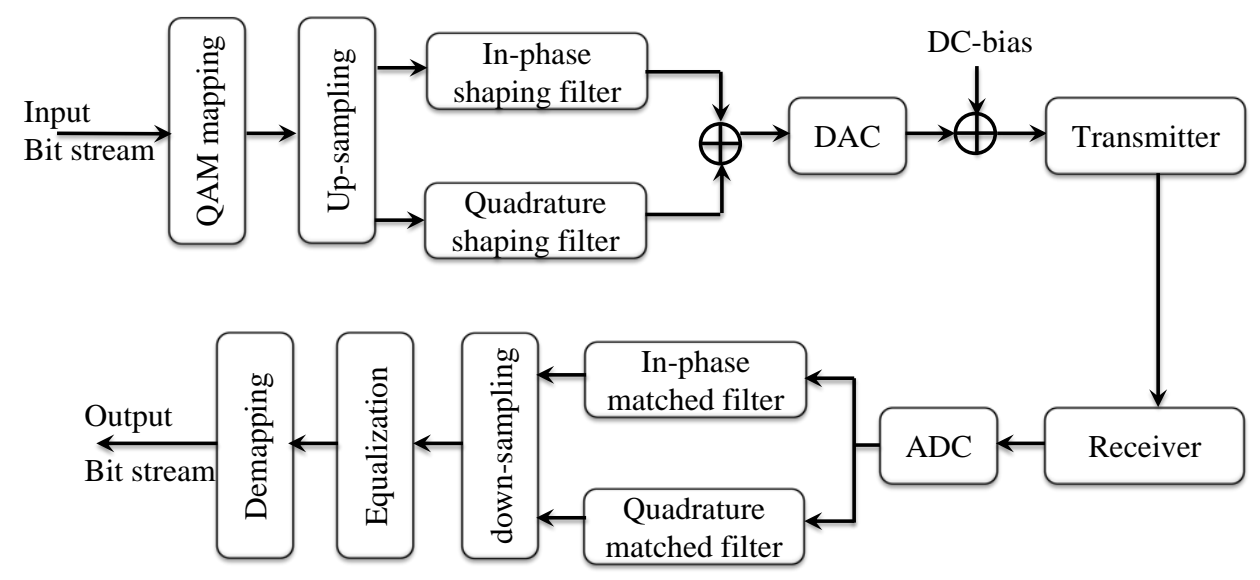

(c)

Figure 12: a) transmitter filter for $I$-channel, b) transmitter filter for $Q$-channel and c) the schematic block diagram of the CAP signal generation and demodulation. 
A schematic block diagram of CAP signals generation and demodulation given in Figure 12 (c). Notice that the CAP modulator and demodulator resembles the traditional QAM system except that the multiplier (multiplying input signal with a carrier signal) is replaced by a bandpass filter [193]. When the carrier frequency is not high, the filters (given in Figure 12 (a) and (b)) can be approximated with a reasonable number of taps. For a high carrier frequency, it is not feasible to approximate the filters [193]. This is the reason CAP is popular for the baseband transmission system [161].

CAP is generated by first mapping the incoming bit stream into a complex QAM constellation, which is then up-sampled by a factor of $N_{s}$, the number of samples per symbol, by inserting $N_{s}-1$ zeros between two symbols. The I and Q sequences are separated and the pulse shaping filters are applied. The outputs of the filers are then summed and converted to analogue signal by an analogue-to-digital converter (ADC). Since CAP signals are bipolar, a DC-bias is added before intensity modulation of the optical transmitter. At the receiver end, two matched filters (matched to I and Q transmitter filters) separate the two orthogonal signals. Then downsampling and signal recovery is carried out, which involves equalization and de-mapping [194].

CAP offers a simpler implementation with much lower PAPR than OFDM [155]. However, for optimum performance, CAP requires a flat-band response. For non-flat spectral systems, CAP requires very complex equalizers [34], [195]. Recently, a multiband CAP was proposed which divides the bandwidth into a number of sub-bands [155]. With a sufficiently large number of sub-bands, each sub-band can be considered to be flat relaxing the condition for a flat-band response. The number of bits/symbol assigned each sub-band can be adjusted as in the case of the bit and power loading OFDM scheme [191], [196], [197]. Multi-band CAP, however, suffers from high PAPR as in the case of other subcarrier modulation [197].

\section{e) Orthogonal frequency division multiplexing (OFDM)}

OFDM is a special case of subcarrier modulation in which the carriers are orthogonal over one symbol period. Since data are transmitted in parallel over a relatively large number of subcarriers, the symbol period for each subcarrier is much longer than for a serial system and, hence, ISI is significantly reduced [174], [172]. Each subcarrier in OFDM is independently modulated and can adapt to match the system frequency response. Hence, OFDM is a preferred option in a band-limited system. Another key advantage of OFDM is its simplicity in implementation as an Inverse Fast Fourier Transform (IFFT) and Fast Fourier Transform (FFT) pair can be used to generate and demodulate the OFDM signal (see Figure 13).

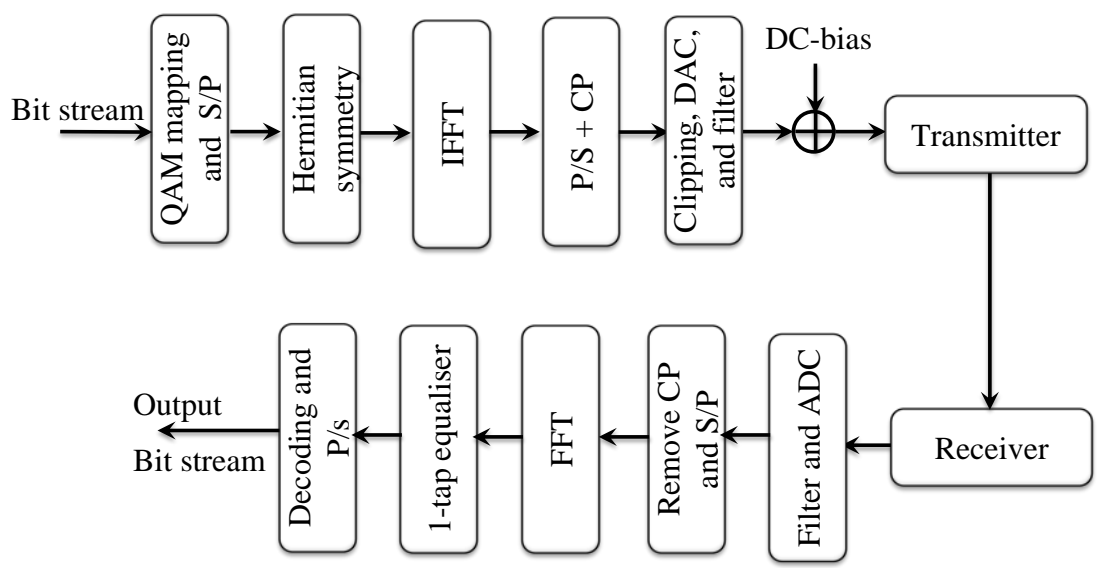

Figure 13: A schematic block diagram of the DCO-OFDM signal generation and demodulation.

Conventional OFDM cannot be directly applied to the IM optical system because the OFDM signals at the output of IFFT (Figure 13) are complex and bipolar and an IM/DD system requires a real and unipolar signal [174]. In order to generate a real signal, Hermitian symmetry is imposed before applying the IFFT i.e. the subcarriers corresponding to the negative frequencies are made complex conjugates of the other half [174]. This reduces the spectral efficiency at least by a factor of two in comparison to conventional OFDM [198], [199]. The real signal is further conditioned to generate a unipolar signal as described below.

In a DC-biased optical OFDM (DCO-OFDM), a Hermitian symmetry is imposed before applying the IFFT to generate a real signal. The output of the IFFT is hard-clipped and a DC-bias is applied to make the signal unipolar. Figure 13 shows the block diagram of DCO-OFDM generation and demodulation processes. 
First, input bit streams are mapped to QAM constellations followed by a serial to parallel (S/P) converter. The signal is then mapped to orthogonal subcarrier frequencies by IFFT. Note that signal on the second half of IFFT inputs is the complex conjugate of the first half, in order to generate real outputs. In most practical OFDM systems, a cyclic prefix (CP) is added to the start of each OFDM symbol i.e. a number of samples from the end of the symbol is appended to the start of the symbol. Though the CP introduces reduces the overall throughput, it eliminates both ISI and inter-carrier interference (ICI). The OFDM symbols are then serialized using P/S, followed by conversion to analogue signals using a digital-to-analogue converter (DAC) and a filter. An appropriate clipping is applied before DC addition to make the signal unipolar. One of the key issues with OFDM signals is their high PAPR [200] and, hence, the OFDM signal needs to be clipped significantly in a dynamic-range-limited system. Even with a high DC-bias, a portion of the negative signal is clipped, thus limiting the performance.

The signal recovery process almost follows the inverse of signal generation. First, the received signal is converted to a discrete time signal by filtering and an analogue-to-digital converter (ADC) followed by $\mathrm{CP}$ removal and serial-to-parallel (S/P) conversion. Then, a FFT is applied to the signal followed by 1-tap frequency domain equalization, parallel-to-serial (P/S) conversion and finally the symbol recovery.

In asymmetrically clipped optical OFDM (ACO-OFDM), the signal is clipped at zero level [201] and hence does not require an additional DC-bias. This is achieved by assigning QAM symbols only to odd subcarriers. In this case, noise generated by asymmetrical clipping below the zero level only falls into even subcarriers, and odd subcarriers are not impaired [201]. Thus, by giving up half of the spectrum, ACO-OFDM reduces the need for a DC-bias. This in return improves the optical power efficiency, but at the cost of reduced spectral efficiency.

Further modifications have been proposed to make OFDM more suitable for IM. Unipolar OFDM (UOFDM) [202] and flip-OFDM [203] separate the positive and negative signal of the IFFT output into two separate frames. The polarity of negative amplitudes is inverted to make them unipolar and zeros are padded before or after each sample to differentiate positive and negative samples. U-OFDM and flip-OFDM offer the same spectral efficiency as ACO-OFDM and achieve the same performance in an additive white Gaussian noise (AWGN) channel [204]. Further improvement in spectral efficiencies can be achieved by superimposing different layers of U-OFDM as outlined in [202].

Azhar et al.[205] performed an experimental comparison of different OFDM schemes. It was concluded that in practical systems with a limited dynamic range, DCO-OFDM outperforms other OFDM schemes. The inferior performance of ACO-OFDM was among other things primarily attributed to the introduction of baseline wander (BLW) due to clipping at zero level. A similar conclusion was obtained analytically in [206], where it is shown that DCO-OFDM outperforms flip-OFDM in an average optical power and dynamic range limited system.

\subsection{Comparison of Modulation schemes}

The selection of an appropriate modulation scheme depends on a number of factors, most importantly spectral efficiency, power efficiency, complexity, reliability, robustness towards interference and resilience to system non-linearity. Due to a number of non-mutual parameters that affect the optimum performance, it is not straightforward to compare modulation schemes under diverse system constraints. Hence, a general conclusion is difficult to draw and contradictory results may be obtained depending upon the underlying assumptions. This section surveys the performance comparison of different modulation schemes, exclusively based on experimental results. Note that these results need to be interpreted with a caution, as they may not match theoretical predictions due to the difference in the underlying assumptions. For example, most of the theoretical studies are carried in an ideal AWGN without a dynamic range limitation and a nonlinear distortion, thus favouring some modulation schemes that may not be optimal in practice.

The optical power penalties for multi-level PAM schemes with a decision feedback equaliser (DFE) depend on the ratio of bandwidth to the data rate. Theoretical studies [178] showed that OOK requires a lower optical power than multi-level PAM if the data rate is less than three times the bandwidth. If the LED is modulated beyond three times the bandwidth, multi-level PAM performs better than OOK. The performance of multi-level PAM schemes, however, degrades in the presence of system non-linearity and hence most of the practical work tends to concentrate on a maximum PAM level of $L=16$. The simulation and experimental studies carried out by Li et al. showed that 4-PAM offered the best receiver sensitivity in comparison to 2-PAM and 8PAM [125] i.e. 4-PAM requires the lowest received optical power to achieve the desirable error rate. Similar conclusions were reached for MIMO-VLC systems [207] and for high-speed polymer optical fibre applications as well [180]. 
Among the huge number of variations of OFDM that have been applied to VLC, ACO-OFDM, PAMDMT, flip-OFDM, U-OFDM and enhanced U-OFDM offer equivalent spectral efficiencies and performance in an AWGN channel [204]. ACO-OFDM offers higher power efficiency than DCO-OFDM for an AWGN channel. However, in a severely bandwidth-limited system, ACO-OFDM is disadvantageous as it wastes half of the spectrum. Similarly, in a dynamic range limited system, the ACO-OFDM signal needs to be clipped at the upper level as well. Unlike zero level clipping, the noise generated by upper-level clipping falls in both the odd and even subcarriers. Similarly, system non-linearity generates harmonics which again affect both the even and odd subcarriers. These effects will degrade the performance of ACO-OFDM. Moreover, the spectrum of ACOOFDM has a large DC component [176] and in an AC-coupled system, this will cause significant baseline wander. Considering all these issues that arise in a practical system, it is not unanticipated that DCO-OFDM outperforms other OFDM schemes in a bandwidth and dynamic-range limited VLC system [37], [205], [206]. However, by using pre-equalization and using twice the bandwidth of DCO-OFDM, it was shown in [208] that ACO-OFDM required $1.5 \mathrm{~dB}$ less optical power than DCO-OFDM to achieve same data rate.

PAM, CAP and OFDM depend on the non-linearity, dynamic range, available SNR, and on the ratio of bandwidth to the data rate. At the high bandwidth to data rate ratio (i.e. low ISI), PAM schemes tend to offer the best performance, whereas at a low ratio (i.e. high ISI), OFDM tends to offer the best performance [178], [209]. A performance evaluation of OFDM and PAM is carried out in [210] for an intensity modulated optical communications system. The authors have considered nonlinear distortion caused by signal clipping and the limited resolution of the digital-to-analogue converter. Based on the analytical results, and confirmed by Monte Carlo simulations, the paper concluded that PAM modulation schemes outperform OFDM and require $3-3.5$ $\mathrm{dB}$ less optical power for bit resolutions of less than four. The experimental results demonstrated in [180] also highlighted that PAM outperforms OFDM. Stepniak et al. carried out theoretical comparisons of a number of modulation schemes for an indoor VLC system in [211] and studied these experimentally in [212]. The theoretical investigation in [211] indicates that among PAM with DFE, CAP with DFE and DMT with bit and power loading, PAM with DFE offers the best performance. Similar conclusions were reached when the comparisons of these modulation schemes were carried out experimentally for a VLC system with a white LED [212]. It has also been shown that 2-PAM and 4-CAP exhibit improved immunity to nonlinear distortions compared to their higher level counterparts. Note that the comparison of these modulation schemes was carried out at modest data rates of $300 \mathrm{Mbit} / \mathrm{s}$ and $450 \mathrm{Mbits} / \mathrm{s}$, which is significantly lower than the maximum data rates demonstrated using white LEDs. The performance of OFDM and CAP was also assessed in [155] using a single colour LED as well as a WDM system with RGB LEDs. In this work, an optimal bit and power loading technique was adopted for OFDM, and pre-emphasis and post equalization was adopted for the CAP. The maximum data rates achieved using CAP for both single and multi-colour systems were higher than those obtained with OFDM. These studies clearly indicate that, unlike in RF, OFDM is not always the optimum modulation scheme for IM optical communication. Nonetheless, most of the world record data rates for VLC systems have been achieved using a single colour or RGB LEDs with bit and power loading OFDM [18], [25], [30], [133].

\subsection{Equalization techniques}

Most VLC links are intrinsically line-of-sight (LOS). LOS link channels generally have high bandwidth in excess of GHz. However, the transceiver elements (especially white LEDs) impose a significant bandwidth bottleneck for high-speed communications, because (as discussed above) commercial white LEDs have a bandwidth of only a few MHz and the blue LED itself has a limited bandwidth of 10's of MHz. Though recently introduced GaN $\mu$ LEDs have a larger bandwidth up to 100's of MHz (as described in Section 3) [23], this is still only a fraction of the targeted data rate in the Gbit/s range. Hence, a number of techniques have been devised and studied to mitigate limitations of the modulation bandwidth. Equalization is a key technology to increase the effective bandwidth by applying a filter that has a frequency response inverse to the system response. Equalization can be analogue or use discretized time, and it can be applied in VLC systems either at the transmitter (pre-equalisation) or at the receiver (post-equalisation). These equalization techniques are discussed in the following sections.

\section{a) Analogue equalization}

The frequency response of an LED can be modelled using a first order low pass filter (LPF) [83] i.e. there is a $20 \mathrm{~dB} /$ decade increment in the attenuation for the frequencies above the $3 \mathrm{~dB}$ bandwidth. This rapid fall-off in the response can be compensated by the introduction of an analogue filter bank, with a combined response which is the inverse of the LED response. One of the simplest techniques to compensate the roll-off is using passive RC high-pass filters with different cut-on frequencies and applying weighted summing to the output of the filters [170]. Figure 14 showed an illustration bandwidth improvement using a simple analogue pre-equalization. Using a simple passive RC pre-emphasis, Burton et al.achieved an overall system bandwidth 
of $10 \mathrm{MHz}$ for a white phosphor LED [213]. Le Minh et al. achieved a tenfold increment in the bandwidth, from $\sim 2.5 \mathrm{MHz}$ to $25 \mathrm{MHz}$, by applying analogue resonant circuits with different central frequencies to 16 independent LEDs and combining their outputs. By using a similar technique, a bandwidth of $45 \mathrm{MHz}$ was achieved for the filtered blue response and an $80 \mathrm{Mb} / \mathrm{s}$ short range data link has been demonstrated [22].

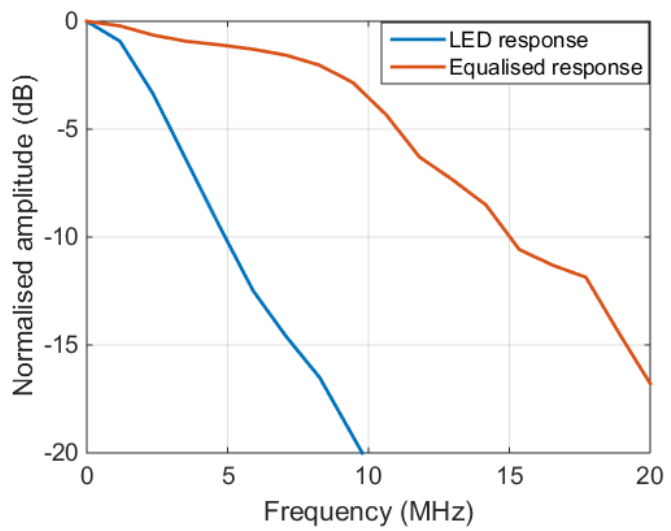

Figure 14: Frequency responses of a typical LED without and with an analogue pre-equalization. There is a more than fourfold increase in the equalized bandwidth.

Using active components, Li et al. demonstrated an analogue pre-emphasis circuit which extended the blue spectrum $-3 \mathrm{~dB}$ bandwidth from 12 to $77 \mathrm{MHz}$ [214]. They further improved the bandwidth up to $233 \mathrm{MHz}$ using multi-stage pre-emphasis based on NPN transistors, enabling data transmission of $550 \mathrm{Mb} / \mathrm{s}$ using OOKNRZ [147], [215] and $682 \mathrm{Mb} / \mathrm{s}$ using 16-QAM-OFDM [139]. Fujimoto et al. proposed a practical LED driver with a pre-emphasis circuit based on a current mode logic (CML) circuit, emitter follower, and RC network and demonstrated a data rate of $662 \mathrm{Mb} / \mathrm{s}$ using a blue LED [151], [163], [216]. Kishi et al. demonstrated an LED driver that sweeps out the remaining carriers in the LED when the LED is going into an off-state [217]. Predistortions are also adopted for other modulation schemes including CAP and OFDM [34], [133], [134], [136], [138], [218].

For VLC applications, post-equalization (equalizer at the receiver) is often the preferred option, although, for a linear system, similar performance can be achieved by pre- and post-equalization. If the primary role of the visible LEDs is to provide illumination then high current driver circuits need to be used to provide adequate illumination. If pre-equalization is adapted, the equalizer needs to be designed such that it can withstand a current of hundreds of milliamps, which is very challenging. If multiple sources are used to provide a high level of illumination or coverage, modification needs to be made for each individual source, thus making the system expensive. The limited dynamic range and non-linearity of the sources also need to be taken into account for optimum equalization. Moreover, the pre-equalizers (based on RC high pass filter) suppress low-frequency components leading to a lower modulation depth and reduced SNR. Post-equalizers are free from these constraints. However, post-equalization enhances noise and pre-equalization can offer a better receiver sensitivity [125].

Using an RC-network based on a first-order analogue post equalizer, Le Minh et al.improved the LED bandwidth from $14 \mathrm{MHz}$ to $50 \mathrm{MHz}$ and achieved a $100 \mathrm{Mb} / \mathrm{s}$ link using OOK-NRZ modulation schemes [83]. Using a post-equaliser based on two passive and one active equaliser, Li et al.extended the LED bandwidth by more than 50 times the raw bandwidth and demonstrated a $340 \mathrm{Mb} / \mathrm{s}$ VLC link [146]. Simple RC-based post equalizers were investigated for a number of modulation schemes in [182], [219], [220]. The pre- and postanalogue equalizations were combined to further improve the data rate beyond $500 \mathrm{Mb} / \mathrm{s}$ [147], [216].

\section{b) Discrete-time equalization}

Discrete-time equalization is typically preferred over analogue equalization as the former offers improved performance [221] and it can be implemented in any modern digital signal processing (DSP) platform, making it easy to adapt to specific applications. Since the ISI caused by limited bandwidth system response is limited to a finite number of symbols, the discrete-time equalizer can be realized based on a finite impulse response (FIR) digital filter. If a system is time-invariant and the response is known in advance, the filter coefficients can be predefined. In a time-variant system, the filter coefficients are adjusted dynamically by transmitting a training sequence in a regular interval.

Discrete-time equalizations are classified into either linear equalizers or decision feedback equalizers (DFEs). A linear equalizer is constructed with a transversal filter that contains delay taps and weighting 
coefficients $\left(C_{n}\right)$ on each tap (see Figure 15(a)). For zero ISI at the sampling instant, the frequency response $G_{e}(f)$ of the equalizer should be an inverse of the system impulse response $c(f)$ [222] i.e.

$$
\mathbf{G}_{e}(\boldsymbol{f})=\frac{\mathbf{1}}{\mathbf{C}(\boldsymbol{f})} .
$$

This class of filter is called the zero forcing equalizer (ZFE) as the ISI is zero at the sampling instant. A major drawback of the ZFE is that it ignores the presence of additive noise. This results in a significant noise enhancement. An alternative is the minimum mean-square error (MMSE) criterion which relaxes the zero ISI condition. The MMSE concurrently minimizes the ISI and noise, giving an optimum performance in the presence of AWGN.

A decision-feedback structure completely removes ISI caused by the previously detected symbols by feeding them back using a feedback structure (see Figure 15(b)). If the current symbol is detected correctly, then the ISI contributed by this symbol in the future received signal can be accurately predicted and removed. The DFE offers significantly improved performance in comparison to ZFE, especially for a severely band-limited system [178]. In fact, the performance of the DFE is close to that of the optimal maximum likelihood sequence detection (MLSD) technique [223].

As in the case of analogue equalization, both pre- and post-equalizations have been studied for VLC applications. Li et al. [125] investigated discrete-time feed-forward pre- and post-equalizations with various numbers of filter taps for PAM schemes. The simulation results showed that the pre-equalization outperforms post-equalization and an improvement of up to $5.5 \mathrm{~dB}$ in receiver sensitivity was obtained for OOK-NRZ. An error-free $2 \mathrm{~Gb} / \mathrm{s}$ VLC link was also demonstrated using a $\mu$ LED and APD receiver. By adaptively changing the pre-equalizer filter coefficients, Yeh et al. improved the system bandwidth using a linear pre-equalizer and achieved $20 \mathrm{Mb} / \mathrm{s}$ using an LED with a bandwidth of $1 \mathrm{MHz}$ [224]. Haigh et al. applied an adaptive linear equalizer and demonstrated a VLC link with a data rate of above $100 \mathrm{Mb} / \mathrm{s}$ using the blue spectral component of a white LED with a bandwidth of $8 \mathrm{MHz}$ [225].

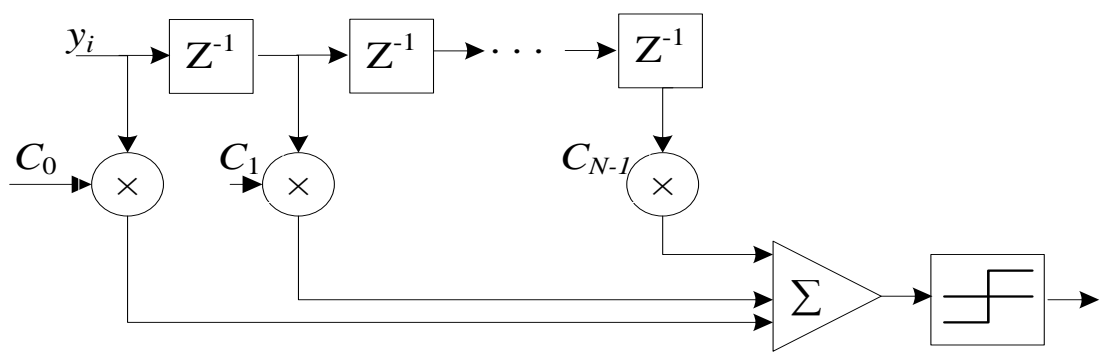

(a)

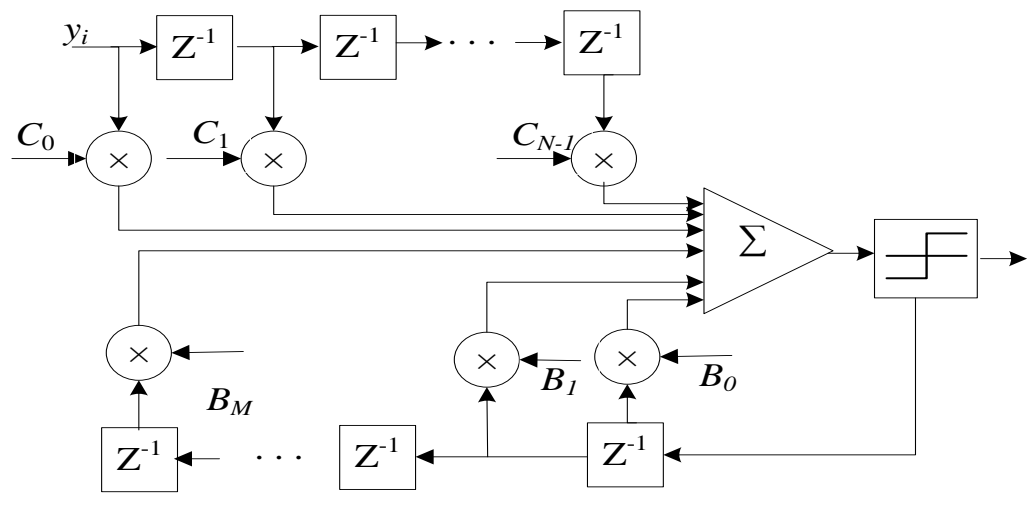

(b)

Figure 15: Schematic of time-domain a) linear equalizer and b) decision feedback equalizer.

A theoretical study showed that DFE significantly outperforms a linear structure without significantly higher complexity [178], which was verified practically in [153]. As a result, most of the high speed VLC 
demonstrations to date use DFE [137], [153], [155], [161], [226] often in combination with analogue preequalization [34], [155]. A fractionally spaced blind DFE algorithm was investigated in [227] in combination with OOK-NRZ and demonstrated a $10 \mathrm{Mb} / \mathrm{s}$ link using an LED with $1 \mathrm{MHz}$ bandwidth. DFE was also successfully applied to CAP to demonstrate a multi-Gb/s VLC link [154], [161]. A complex DFE is adopted in these reports and is applied to the in-phase and quadrature-phase channels of CAP.

Typically, LEDs have non-linear impulse responses. FIR-filter-based equalizers are not optimal for the compensation of ISI in a non-linear system [228], [229]. Rajbhandari et al. studied an artificial neural network (ANN) based receiver for compensating ISI and non-linear response of VLC systems and practically demonstrated that the ANN outperforms filter-based equalizers [152], [153]. For example, a data rate of $170 \mathrm{Mb} / \mathrm{s}$ was achieved using a decision feedback ANN structure for OOK-NRZ. For the identical link, only $90 \mathrm{Mb} / \mathrm{s}$ was feasible with DFE, demonstrating a clear advantage of ANN for the VLC link. However, the computational complexity of the ANN-based receiver is significantly higher than DFE, as the ANN employs non-linear transfer functions to realize a non-linear response. Alternatively, a Volterra-based nonlinear DFE was adapted to compensate the LED non-linearity and ISI for PAM and CAP modulation schemes [34], [130], [137], [230]. In [230], a non-linear DFE structure was realized with a second order nonlinear Volterra feed-forward section. It was demonstrated that the Volterra DFE can efficiently compensate LED nonlinearity and can improve the receiver sensitivity by up to $5 \mathrm{~dB}$ for multilevel PAM. A data rate of $1.1 \mathrm{~Gb} / \mathrm{s}$ was accomplished using the symbol-spaced Volterra DFE for the PAM scheme [137]. Due to the non-linear response, the Volterra DFE requires a significantly longer training time as the convergence is slower than in the case of traditional DFE [230]. In order to reduce the training duration, Wang et al. proposed a modified cascaded multi-modulus algorithm (M-CMMA) which updates the filter weights of the nonlinear equalizer without using training symbols [130]. An aggregate data rate of $4.5 \mathrm{~Gb} / \mathrm{s}$ was achieved with RGB LEDs by compensating the nonlinearity with the Volterra DFE using M-CMMA training. They further improved the data rate up to $8 \mathrm{~Gb} / \mathrm{s}$ using a hybrid equalizer consisting of a linear, a Volterra-series-based nonlinear, and a decision-directed least mean squares equalizer [34]. Non-linear compensation techniques using pre-distortion and post-distortion are further outlined in [231]-[233].

Frequency domain equalizers (FDE) have recently also been explored for VLC systems [143], [145], [234]-[237]. In the FDE approach, a fast-Fourier-transform (FFT) is performed on a block of symbols before applying a single-tap equalizer in the frequency domain as in the case of the OFDM. The combination of PAM with block transmission and FDE has potential to offer improved performance over PAM-DMT and OFDM [237], [238]. Kashani et al. studied the performance of Nyquist single carrier FDE (NS-CFDE) for VLC [239]. The NS-CFDE has a similar spectral efficiency as DCO-OFDM but a significantly reduced PAPR. Hence, NSCFDE has potential to outperform OFDM and OOK schemes, especially for dynamic range limited cases [239]. Wang et al. applied NS-CFDE [145] experimentally and demonstrated a data rate of 4.22-Gb/s for RGB LEDs with WDM. NS-CFDE was also studied for a $2 \times 2$ MIMO system [143] where MIMO de-multiplexing and postequalisation were employed in the frequency domain.

The OFDM scheme uses a single tap FDE to compensate for ISI. OFDM-VLC demonstrations often apply an analogue pre-equalization and frequency domain post-equalization [134], [138]. Xingxing et al. [130] applied a cascaded analogue pre-equalizer to increase the $-3 \mathrm{~dB}$ bandwidth of commercial white LEDs from 17 $\mathrm{MHz}$ to $366 \mathrm{MHz}$, and a data rate of $1.6 \mathrm{~Gb} / \mathrm{s}$ was demonstrated using 16-QAM-OFDM. In [25], the performance of DCO-OFDM with pre-equalization and post-equalization was investigated for a blue $\mu$ LED and it was demonstrated that combining pre- and post- equalizations offers an improved performance rather than relying only on the post-equalization. The optimum performance, however, was obtained by applying an adaptive bit and power loading [25]. In sub-carrier modulation schemes like OFDM, the constellation level and the assigned power level in each sub-carrier can be independently adjusted depending upon the available SNR, thus optimizing the achievable data rates. Using adaptive bit and power loading, a blue $\mu$ LED based VLC link achieved $3.22 \mathrm{~Gb} / \mathrm{s}$ in comparison to $2.63 \mathrm{~Gb} / \mathrm{s}$ using combined pre- and post-equalization [25]. Similarly, for a VLC link based on a single phosphorescent white LED with a blue filter, a data rate of $2 \mathrm{~Gb} / \mathrm{s}$ was achieved using adaptive bit and power loading in [133] whereas the same link offered only $1.6 \mathrm{~Gb} / \mathrm{s}$ when incorporating analogue pre-equalization with post equalization. These results clearly demonstrated that OFDM with bit and power loading maximize the system capacity and, as a result, most of the high-speed VLC demonstrations have employed adaptive bit and power loading [18], [25], [30], [159], [240]. The performance of OFDM also suffers due to its high PAPR and susceptibility to a nonlinear system response. The effect of non-linearity can be reduced by limiting the PAPR by pre/post distortion. In [200], the authors recommended applying an inverse nonlinear polynomial of the LED I-V response to a signal before intensity modulating the LED so that the overall system response is a linear function. A hybrid time-frequency domain equalization that compensates ISI and nonlinear distortion for OFDM was experimentally demonstrated in [240]. In this case, ISI was compensated by FDE and the nonlinear distortion by an adaptive nonlinear-Volterra-based time domain 
equalizer. Qian et al. proposed an adaptive post-distortion for compensating the non-linear response of LEDs [232]. The authors applied a memory polynomial model to track and compensate the LED's nonlinearity.

\subsection{Spatial Multiplexing/Modulation}

VLC systems can take advantage of the availability of multi-element, multi-coloured illumination devices to improve coverage, data rate and the reliability of the communications system by exploiting spatial multiplexing and WDM. This section provides an overview of spatial modulation/multiplexing and WDM is introduced in the next section.

There are a number of ways to drive the spatially separated LEDs required, which can be broadly classified into:

a) A 'ganging' approach

b) A multiple input multiple output (MIMO) approach

\section{a) Ganging Approach}

In the ganging approach, also known as repetition coding [241], multiple LEDs are modulated using the same data source and spatially superposed as shown in Figure 16. The individual LEDs can have an individual driver with all drivers receiving the same data. Alternatively, the LEDs can be connected in series or parallel and driven by a single driver. Multiple LEDs are ganged together in order to provide a better link margin, wider coverage or increased resilience to blocking of the path between LED and receiver. In [170], 16 white LEDs were driven by 16 drivers to provide an improved SNR at the receiver and a data rate of $40 \mathrm{Mb} / \mathrm{s}$ was achieved using OOK-NRZ. In [242]-[244], increased area coverages were achieved using a number of transmitters each of which had a limited divergence angle and pointed in a different direction. Increased angular diversity can be achieved by a planar array of LEDs in combination with optics as in [244] or by arranging LEDs in a semispherical base with each LED pointing in a different direction [242], [243].

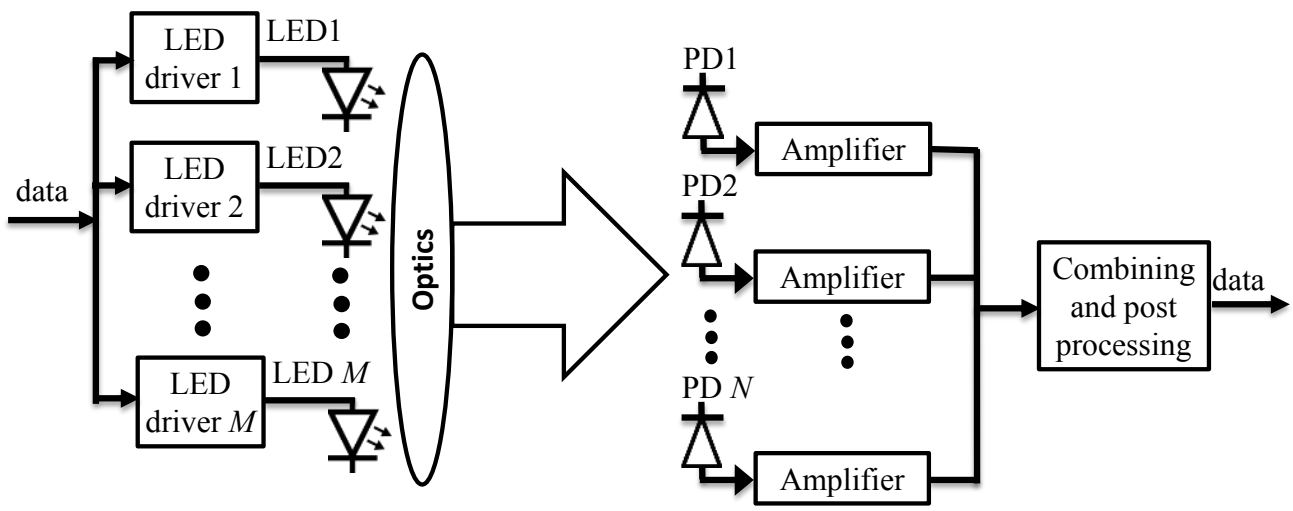

(a)

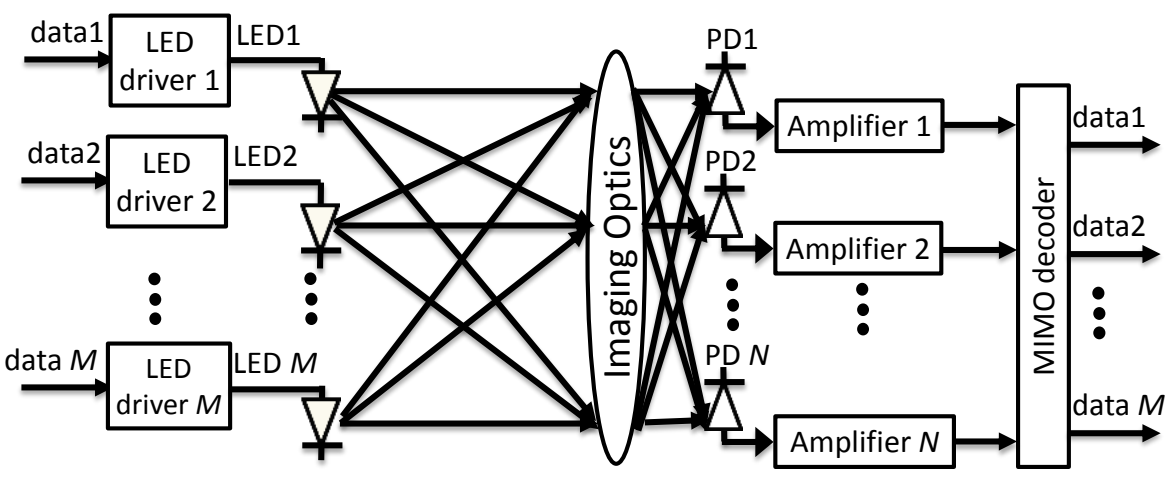

(b) approach.

Figure 16: Approaches to drive multiple LEDs using an individual driver a) ganging approach and b) MIMO 
The spatial diversity can be applied to the receiver by different diversity combing algorithms which include equal gain combining (EGC), maximum ratio combining (MRC) and selection combining (SelC). In the EGC spatial diversity technique, the photocurrents from all receivers are summed giving equal weight to them. In the MRC techniques, a weight proportional to the received optical power is given to each receiver before linearly combining the photocurrent. In the SelC algorithm, the receiver with the highest SNR or largest photocurrent is selected disregarding other receivers. Hence, the output is equal to the signal on only one of the receivers and is not the linear sum of the individual photocurrents. As the MRC algorithm requires the estimation of the received optical power for each receiver, it is the most complex algorithm. Nonetheless, MRC is often the optimal spatial combing algorithm [245]. However, a simple selection combing scheme which detects the best channel based on received signal amplitude is often the adequate making the receiver design simple [242].

In [246], [247], spatial diversity receivers with MRC were considered for a single input multiple output (SIMO) system reducing the BER by one to two orders of magnitude. The coverage and mobility of a VLC system were improved by applying SelC in conjunction with angle diversity receivers [248].

\section{b) MIMO approach}

The link capacity of a communications system is governed by Shannon's channel capacity formula i.e.

$$
C=B \log _{2}(1+S N R)
$$

where $C$ is the channel capacity (in bits/Hz), $B$ is the bandwidth.

In the ganging scheme, the SNR at the receiver increases linearly with the number of transmitters. However, based on (6), the system capacity increases only logarithmically with the SNR. The MIMO scheme, on the other hand, has the potential to linearly increase the capacity with the minimum number of the transmitters and receivers by parallel transmission of data. A simplified schematic of the MIMO scheme for VLC systems is shown in Figure 16(b) with $M$ transmitters and $N$ receivers.

The received signal at any discrete-time instance $k$ for a MIMO system is given by:

$$
\mathbf{y}(k)=\mathbf{H x}(k)+\mathbf{n}(k)
$$

where $\mathbf{x}(k)$ is a $(M \times 1)$ transmitted signal vector, $\mathbf{y}(k)$ is a $(N \times 1)$ received signal vector, $\mathbf{n}(k)$ is a $(N \times 1)$ noise vector and $\mathbf{H}$ is a $(N \times M)$ channel matrix.

The channel $\mathbf{H}$-matrix is given by:

$$
\mathbf{H}=\left[\begin{array}{cccc}
h_{11} & h_{12} & \cdots & h_{1 M} \\
h_{21} & h_{22} & \cdots & h_{2 M} \\
\vdots & \vdots & \ddots & \vdots \\
h_{N 1} & h_{N 2} & \cdots & h_{N M}
\end{array}\right]
$$

where $h_{i j}$ is the channel gain from the $j^{\text {th }}$ transmitter to the $i^{\text {th }}$ receiver elements. For a MIMO system to operate optimally, the channel $\mathbf{H}$-matrix must be full-rank. For the channel $\mathbf{H}$-matrix to be a full-rank in an IM/DD system, the number of receivers should be larger or equal to the number of transmitters, i.e. $N \geq M$ [170], [249].

There are a number of MIMO techniques for VLC systems. The unique features and advantages of these individual techniques are reviewed in the following section.

\section{4.a.1 Spatially superposed modulation}

In the spatially superposed modulation scheme, the signal at the receiver plane is obtained by superposing the optical signals from the different transmitters. In superposed PAM (SPAM), the individual transmitters transmit simple 'ON-OFF' data which are weighted and superposed to obtain the desired multilevel PAM [182], [250]-[252]. In superposed OFDM, the individual LEDs transmit fractions of the subcarrier bands of an OFDM symbol which are overlaid in free space [253], [254]. The superposed OFDM reduces the PAPR per LED and hence reduces the effect of non-linear clipping. Alternatively, the OFDM signal amplitude can be iteratively clipped to ensure that the signal falls within the linear region of the individual LEDs and 
transmitted using different LEDs as outlined in [255]. In both OFDM schemes, the required dynamic range of LEDs is significantly reduced.

SPAM can be generated by ganging a different number of LEDs together and driving them with an equal intensity [252] or by independently adjusting the modulation current of individual transmitter elements [182]. A phase-shifted OOK scheme with multiple LEDs was studied in [256]. The parallel streams of data from different LEDs with an appropriate delay were optically superimposed. Using a high sampling rate at the receiver with phase shift information, the individual data streams were recovered. It is shown that the overall data rate can be improved linearly with the number of LEDs using phase-shifted OOK. In [253], [254], an OFDM signal was divided into a number of narrowband OFDM signals which were transmitted simultaneously using different LEDs.

SPAM has an advantage over multi-level PAM because SPAM can be generated by a simple 'ON-OFF' modulation of transmitters. This, in turn, avoids LED nonlinearity issues, and reduces the driver complexity as DACs are not required. In fact, this is equivalent to an optical DAC [257]. A narrowband subset of an OFDM signal has a significantly lower PAPR than the overall OFDM signal. Hence, the superposed OFDM transmitter requires a lower dynamic range and the effect of non-linearity in the performance is significantly reduced. In fact, it was shown that spatially superposed optical OFDM outperforms other variations of OFDM [253], [254].

\section{4.a.2 Spatial multiplexing}

The spatial multiplexing (SMP) technique relies on a number of transmitters each carrying an independent data stream to enhance the spectral efficiency. It was shown that the capacity of SMP is proportional to the minimum of transmitters $N$ and receivers $M$ [258]. SMP has been extensively studied in RF communications where arrays of antennas with a spacing larger than half the wavelength $\lambda / 2$ were used to provide significantly de-correlated copies of signals at the receiver. Owing to the shorter wavelength, the transmitter elements' separation and the receiver elements' separation can be in the millimetre range for the channel decorrelation in VLC systems. Hence, a massive MIMO within a tightly confined space is achievable in VLC. For example, a massive MIMO using a $512 \times 512$ pixel liquid crystal display panel and $154 \times 154$ pixels of a charge-coupled device camera was demonstrated and substantially increased the overall data rate [32]. In a non-imaging MIMO system, the receiver relies on the MIMO decoding algorithm to de-correlate the channels, whereas in so-called 'imaging MIMO' optics reduce the inter-channel cross-talk by creating an image of the source array on a detector array [170]. Detailed considerations for the design of imaging and non-imaging optics for a MIMO system is given below in section $4.4 \mathrm{~d}$.

\section{4.a.3 Space shift keying/Colour shift keying}

Space shift keying (SSK) [259], also known as optical spatial modulation (OSM) [35], combines the spatial multiplexing with a digital modulation technique for high spectral efficiency [241]. SSK activates only one of the $M$ spatially separated transmitters at a time. The symbol information is then carried by the index of the activated transmitter. Figure 17 shows a block diagram of SSK with four transmitters. Depending upon the incoming data stream, one of the $M$ LEDs is active during one symbol duration. In the illustration in Figure 17, an incoming 2-bit symbol of ' 00 ' actives the LED1, '01' actives LED2 and so on. Each active transmitter, in this case, encodes $\log _{2}(M)$ bits of information and, therefore, the spectral efficiency is increased by a factor of $\log _{2}(M)$. Unlike SMP, SSK completely avoids inter-channel interference (ICI) because only one LED is active at any given time. This simplifies the decoding algorithm and reduces the required number of receivers. Further improvement in spectral efficiency up to $M$ bits/symbol can be achieved by adopting generalized SSK (GSSK) in which multiple LEDs can be active during one symbol duration [260], [261]. SSK has also been applied in conjunction with optical OFDM to improve the throughput [262], [263].

Similar to SSK, colour shift keying (CSK) exploits the wavelength dimension of optical radiation rather than the spatial dimension. The IEEE 802.15.7 standard adopted CSK for VLC. In CSK, the information is encoded by varying the intensity of the individual components of a red-green-blue light source and decoded by exploiting the wavelength dependence of the receiving silicon photodiode (PD). The differentiability of SSK channels can also be improved by employing different wavelength LEDs at different locations [264]. 


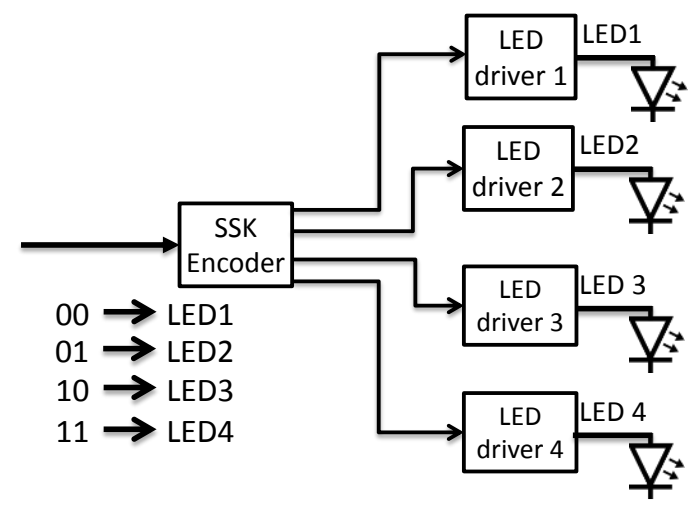

Figure 17: Schematic of a space shift keying transmitter, in this case with four LEDs.

c)

Optics systems for MIMO receivers

The optical power available at the receiver is proportional to the effective collection area. Increasing the photodiode area not only tends to be expensive but also reduces the bandwidth as the photodiode intrinsic capacitance, which governs the receiver bandwidth, depends on its area. Hence, it is essential that a high-speed communications system incorporates a small-area photodiode. In order to keep the photodiode area small without sacrificing the link margin, an optical concentrator is employed at the receiver. Typically, the concentrator comprises a set of lenses or concentrators but other implementations, such as e.g. luminescent concentrators, are also possible [265]. The optical concentrator increases the effective receiver area and thus improves link margin, albeit typically at the expense of a reduction of the field of view (FOV). The Conservation of Etendue governs the maximum theoretically achievable optical gain for a specified FOV, as given by [266]:

$$
A_{\text {coll }} \sin ^{2}\left(\frac{F O V}{2}\right) \leq A_{d e t}
$$

where $A_{\text {coll }}$ is the collection area and $A_{\text {det }}$ is the photodiode active area.

In order to achieve an optical gain close to the Etendue limit, both imaging and non-imaging optics have been studied for VLC systems which are described below.

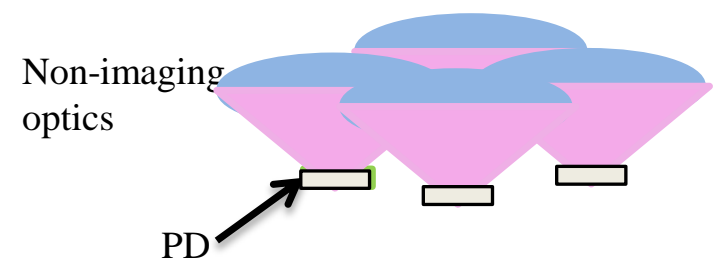

(a)

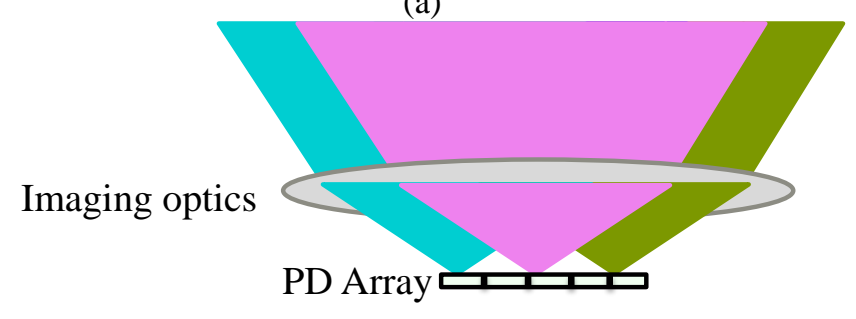

(b)

Figure 18: Schematic of a) non-imaging and b) imaging MIMO optics. Note that different emission colours are used for illustration purpose only and do not represent the wavelength of the light.

A schematic of a non-imaging MIMO receiver is shown in Figure 18 (a). It consists of a number PDs with individual optical concentrators. Hemispherical lenses, converging lenses or compound parabolic concentrators (CPCs) can be used for non-imaging MIMO systems. Although hemispherical lenses can achieve a gain of $n^{2}$ with the FOV $\sim \pi / 2$ [48], this is not suitable for a MIMO system, because most MIMO systems require a high 
gain but a limited FOV. CPCs, on the other hand, can be designed to have a higher gain, however the maximum gain is limited by the Conservation of Etendue [267]. Recently, a new type of non-imaging receiver based on wavelength conversion by a fluorescent material has shown a significant gain (beyond the Etendue limit) without reduction of the FOV [265], [268], [269]. Luminescent concentrators also currently attract much interest for solar energy applications, and it is expected that novel designs developed in this field will prove adaptable to VLC. Examples include mechanically flexible concentrators based on doped polysiloxanes [270], and optical cavity designs in which colour-converted light is trapped using an omnidirectional dielectric mirror [271].

An imaging receiver forms an approximate image of the transmitter elements on an array of receiver elements, as shown in Figure 18(b). In order to successfully separate the MIMO channels, it is not necessary to have perfect one-to-one imaging between the transmitter and receiver elements as long as the image of more than one transmitter does not fall entirely on a single receiver [249]. Imaging MIMO systems can use a singlet convex or Aspheric lens [249] or a combination of lenses [75], [157], [272], [273]. A wide FOV imaging receiver based on a hemispherical lens has been studied in [274], [275]. Hemispherical lenses produce distorted images, but this is not an issue for MIMO systems. An angle diversity MIMO receiver with a Fisheye-lensbased imaging system can provide high FOV and compact receiver structure [141], [276] and is a candidate for a high-speed communications system.

Imaging MIMO offers a number of advantages in comparison to the non-imaging system. The imaging system guarantees a full rank channel $\mathbf{H}$-matrix whereas the channel $\mathbf{H}$-matrix of a non-imaging optical MIMO system can be ill-conditioned for certain transmitter and receiver configurations. An ill-conditioned $\mathbf{H}$-matrix will increase the error probability drastically [170]. In practice though, non-invertible $\mathbf{H}$-matrices have only rarely been observed because manufacturing variations in the transceiver elements aid channel separation [144], [277]. Furthermore, a full rank H-matrix can be guaranteed by using a non-imaging receiver structure with different FOVs [278]. Imaging MIMO receivers are typically more compact and scalable than their non-imaging counterparts. In an imaging system, the entire PD array can share a single optical system which creates a demagnified the image of the transmitter [272], [273]. Moreover, the MIMO photodiode array can be planar which allows fabrication using highly scalable CMOS technology. The downside of imaging MIMO systems is their relatively limited FOV. In order to improve the FOV, imaging MIMO systems require a very large number of receiver elements in the range of 100's to 1000's which increases the computational complexity and cost.

\section{d) MIMO decoding algorithms}

The effect of intra-channel interference (ICI) can be mitigated by implementing channel inversion or equalization at the receiver. Similar to the equalization techniques described above in section 4.3, MIMO equalization can broadly be classified into linear and decision feedback equalization.

In the linear equalization approach, the transmitted signal $\widehat{\mathbf{x}}$ is estimated from the received signal $\mathbf{y}$ by linearly combining the elements of $\mathbf{y}$ with a weight vector, $\mathbf{W}$, i.e. the estimated transmitted signal is given by:

$$
\widehat{\mathbf{x}}(k)=\mathbf{W y}(k)
$$

The simplest linear equalization methods to estimate the transmitted signal are the so-called "zeroforcing" (ZF) and "minimum mean-squared error" (MMSE) algorithms. In the ZF algorithm, the ICI is suppressed by multiplying the received signal with a "pseudo-inverse" of the channel matrix $\mathbf{W}_{Z F}$ as given below:

$$
\mathbf{W}_{Z F}=\left(\mathbf{H}^{H} \mathbf{H}\right)^{-1} \mathbf{H}^{H}
$$

where $\mathbf{H}^{H}$ is the conjugated transpose of $\mathbf{H}$.

The ZF algorithm, however, neglects the presence of noise. A more robust equalizer is based on the MMSE algorithm which jointly minimizes the noise and interference. The pseudo inverse $\mathbf{W}_{M M S E}$ of $\mathbf{H}$ using MMSE is given by [279]:

$$
\mathbf{W}_{M M S E}=\left(\mathbf{H}^{H} \mathbf{H}+\frac{\mathbf{1}}{\operatorname{SNR}} \mathbf{I}\right)^{-\mathbf{1}} \mathbf{H}^{H}
$$

where $\mathbf{I}$ is a $M \times M$ identity matrix.

The ultimate linear algorithm is maximum-likelihood-decoding (MLD) which chooses the most probable transmitted vector out of all possible transmitted vectors based on the knowledge of the channel matrix and a received vector $\mathbf{y}(\mathrm{k})$ i.e. 
Though MLD offers the best possible channel separation, computational complexity increases exponentially with the number of parallel channels and constellation levels.

A non-linear approach, where ICI is cancelled iteratively, offers improved performance compared to the linear approach. A popular non-linear MIMO decoding algorithm is the vertical Bell Labs layered space-time algorithm (V-BLAST). The V-BLAST algorithm employs an estimation and successive interference cancellation strategy i.e. the strongest signal is estimated first, and then the inference from this symbol is removed from remaining symbols. Then the next strongest symbol is estimated followed by interference removal and so on. The V-BLAST algorithm can be considered as a generalized DFE [280].

In a bandlimited MIMO system, both spatial (among MIMO channels) and temporal (inter-symbol) interferences occur. A joint spatial and temporal decision feedback equalization (DFE) as outlined in [281] can be adopted in such system.

\section{e) Recent demonstrations}

Among the MIMO techniques discussed here, SMP offers the best spectral efficiencies, however at the cost of high computational complexity. Also, the performance of SMP is highly dependent upon the channel correlation [241]. A numerical study carried out by Butala et al. showed that SMP outperforms SSK for higher spectral efficiencies (8 bits/sym) whereas SSK offers improved performance at lower spectral efficiencies (4 bits/sym) [282].

There is only limited experimental work published using SSK. A proof-of-concept SSK demonstration using 4 transmitters and 1 receiver achieved a data rate of $18 \mathrm{Mbit} / \mathrm{s}$ using white LEDs [283]. Popoola et al. studied a GSSK scheme which offers a high spectral efficiency [260]. Simulation results and experimental measurements verified that GSSK incurs limited receiver mobility, as the error performance of GSSK depends heavily on the provision of dissimilar channel gain values. SSK is also applied in conjunction with OFDM and a $1.34 \mathrm{~Gb} / \mathrm{s}$ OSM-OFDM was demonstrated in [262] using $\mu$ LEDs in a $2 \times 2$ MIMO configuration. The experimental results showed that OSM-OFDM offers improved data rate in comparison to SISO-OFDM. However, the performance is dependent on the divergence angle of the LEDs. By far the most practical MIMO VLC demonstrations are based on SMP, which is possibly due to its high spectral efficiency [32], [60], [75], [143], [144], [156], [205], [249], [272], [284]-[286]. Low data rate and wide area coverage MIMO systems were mostly demonstrated using non-imaging optics [143], [144], [284], [285], [287] whereas higher data rate and integrated transceiver based demonstrations are preferentially realized with imaging optics [33], [156], [205], [272]. Imaging MIMO systems using standard camera technology were also adopted for low data rate communications [32], [60], [75].

The first demonstration of a non-imaging MIMO VLC system was reported in [284]. Four white LEDs with $0.2 \mathrm{~m}$ pitch simultaneously transmitted independent OOK-NRZ data to four receivers with a $0.1 \mathrm{~m}$ pitch. Non-optimal matrix inversion techniques were used to recover the data and data rates in the range 10-12 Mbits/s were demonstrated without any equalization. Burton et al. demonstrated a $4 \times 4$ non-imaging MIMO-VLC system with a link distance of $2 \mathrm{~m}$, an area coverage of $400 \mathrm{~cm}^{2}$ and a data rate of $50 \mathrm{Mb} / \mathrm{s}$ [144]. Each MIMO channel combined 4 LEDs driven by the same OOK-NRZ data and an analogue pre-equalizer circuit was incorporated to improve the bandwidth. A number of MIMO equalization techniques were also tested and the performance analysis showed that ZF offers similar performance to other computationally complex equalizers like V-BLAST. Haigh et al. used post-equalization using a neural network in combination with ZF demultiplexing and achieved an aggregate data rate of $1.8 \mathrm{Mb} / \mathrm{s}$ using white LEDs and four large area organic photodetectors (without any optics) [285]. Wang et al. used frequency domain equalization as well as simultaneous de-multiplexing and post-equalization to the MIMO signal and demonstrated an aggregate data rate of $500 \mathrm{Mb} / \mathrm{s}$ using a $2 \times 2$ non-imaging MIMO setup [143] and up to $1.4 \mathrm{Gbits} / \mathrm{s}$ using an imaging system [287].

Imaging MIMO systems guarantee a full rank $\mathbf{H}$-matrix if the optics are designed to match the transmitter pitch with the detector pitch [249] and hence offer a significant advantage over non-imaging MIMO [170], [282]. Moreover, imaging optics partially separate the MIMO channels optically, hence allow the use of simpler MIMO decoding algorithms. This makes imaging MIMO alluring for high-speed VLC systems. Azhar et al. demonstrated Gbit/s transmission using a 4×9 imaging MIMO system [157]. A singlet aspheric lens was used to form the image of the transmitter on the receiver array. The number of receiver elements was higher than the number of transmitter elements to provide wide coverage and resilience to vertical and horizontal displacement. Using high bandwidth $\mu$ LEDs, a 1.5 Gbits/s MIMO VLC system with a limited FOV was demonstrated using four parallel channels [156]. Recently, a multi-Gb/s MIMO-VLC system was developed under the UK Engineering and Physical Science Research Council's UP-VLC project [272]. The demonstrator 
used multiple $\mu$ LEDs driven by a custom designed CMOS based current-steering driver, a CMOS based $3 \times 3$ planar APD array with a pitch of $250 \mu \mathrm{m}$ [272] and imaging optics designed to match transmitter and receiver pitch. Photographs of the demonstrator are shown in Figure 19. By using joint spatial and temporal equalization for the 4-PAM scheme, a data rate of $1.3 \mathrm{Gbit} / \mathrm{s}$ was achieved using four channels within the FOV of 3.5 degrees [33] and a data rate up to $7 \mathrm{Gbit} / \mathrm{s}$ was achieved using nine channels at a reduced FOV. Furthermore, MIMO systems using LED displays as a transmitter and a commercial charge-coupled device (CCD) as a receiver have been developed [32], [60], [75]. These demonstrations highlight the feasibility of massive MIMO with a high spatial density.

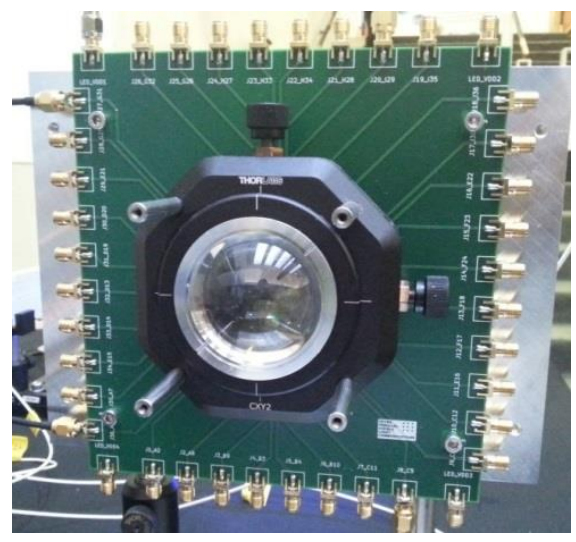

a)

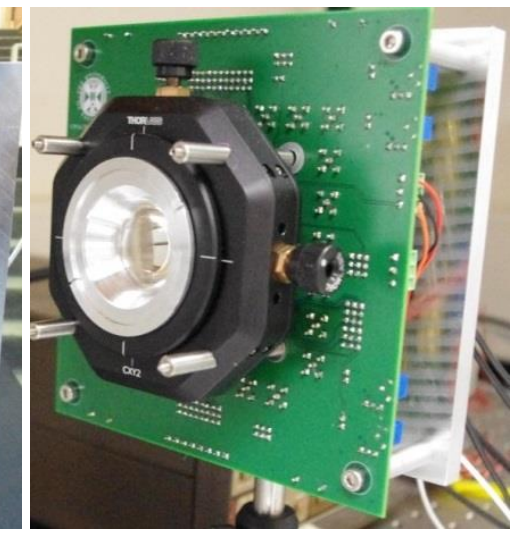

b)

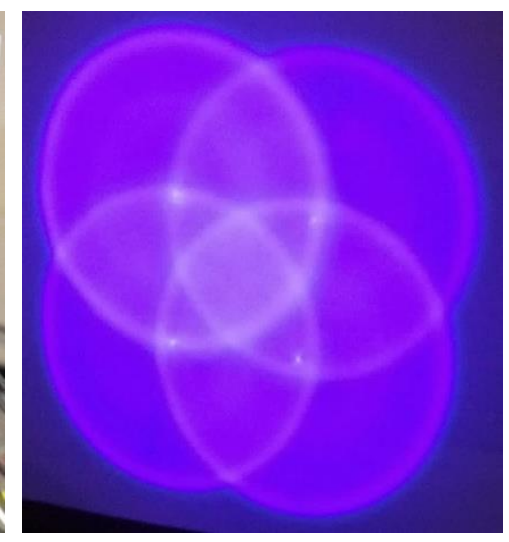

c)

Figure 19: A MIMO VLC demonstrator developed for the UP-VLC project, showing a) the transmitter system, b) the receiver system [33] and c) an image of the $4 \mu$ LED beams as seen at the receiver plane.

\subsection{Wavelength Division Multiplexing}

WDM is a multiplexing technique that allows simultaneous transmission of multi-stream data using different optical wavelengths. The white light suitable for illumination can be generated by mixing a number of narrowband emissions at multiple wavelengths. Naturally, these wavelengths can be exploited to increase the system capacity by independently modulating the individual narrow-band light sources using independent data streams. By concurrently transmitting information over different wavelengths, the system capacity can be linearly increased with the number of wavelengths used.

A schematic of a WDM-based transmitter and receiver is given in Figure 20. The transmitter consists of multiple sources with peak emission at different wavelengths (three in the schematic). The emissions from these sources are mixed together by superposing them in free space or by using an optical system such as a holographic diffuser. At the receiver, these wavelengths are split by optical bandpass filters (dichroic mirrors) and directed towards photodiodes.

WDM in VLC systems tends to be limited to three colours (Red-green-blue) or four colours (Redyellow/amber-green-blue) due to the availability of commercial devices with these wavelength components that were originally developed for illumination. Due to the relatively broad spectral full width at half maximum (FWHM) of LED emission (typically 25-100 nm), dense WDM is not feasible with current LEDs. Using lasers with narrow FWHM (typically $<5 \mathrm{~nm}$ ), a significantly larger number of WDM channels is practicable, enabling the feasibility of 100's of Gbits/s VLC systems [31]. Instead of using multi-colour LEDs, the authors in [38] applied single coloured GaN $\mu$ LEDs and polymer colour-converters to achieve emission at different wavelengths. The advantage of this approach is the possible cost reduction as a single semiconductor die with different polymer colour-converters deposited on the top can be used to generate white light rather than packaging multiple LED die together. Moreover, the green/yellow-emitting LEDs are still relatively inefficient compared to blue/red LEDs. Hence, the blue LED/polymer approach may be more efficient of generating multiple wavelengths. There have been a number of WDMWDM-VLC demonstrations using different modulation schemes [30], [34], [129], [130], [164], [165]. The earliest adaptation of WDM in a VLC system was reported in [165] using RGB LEDs. The authors optimized the capacity of each channel using DMT modulation with a bit and power loading QAM constellation. Cossu et al. adopted a similar approach of optimizing each channel by using bit and power loading with OFDM. They demonstrated an aggregate data rate of 3.4 Gbits/s using RGB LEDs [159], [160] and 5.6 Gbit/s using a red-blue-green-yellow (RGBY) LED [30]. 
Wang et al. also demonstrated a 4.5 Gbits/s system using an RGB LED [130] and an 8 Gbits/s VLC system using an RGBY LED [34] with CAP modulation and a Volterra-based non-linear equaliser. Chun et al. studied the impact of the different colour combination on the communications system and theoretically established the maximum achievable data rate for different RGB colour combinations as a function of CIE colour coordinates [18]. The authors also practically demonstrated a $\sim 11.3 \mathrm{~Gb} / \mathrm{s}$ VLC system using blue and green $\mu$ LED and red RCLED.

The demonstrations reported in [30], [130], [159], [160], [165] used a single receiver and optical filters were used to select a particular channel at a time. Though this approach provides a capacity estimation for WDM, this is far from being practical, as the practical system will require the simultaneous reception of all the channels. Chun et al. overcame this issue by adopting reflective dichroic filters as shown in Figure 20 [18] thus enabling simultaneous reception of the entire channels. However, the practical implementation of such a system is challenging, as it has FOV constraints and is difficult to miniaturize. An alternative colour de-multiplexer based on holographic gratings to separate RGB light and focus each colour onto different directions was studied in [288]. Holographic-based de-multiplexers tend to be more compact than filter and lens based WDM receivers. The best alternative for simultaneous reception of WDM channels is possibly to use a planar single photodetector array with plasmonic colour filters. As outlined in [289], three primary colours can be effectively separated by plasmonic colour filter integrated on a CMOS photodiode. Colour filtering was achieved using surface plasmon resonance (SPR) in thin metal films and can easily be realized in a conventional CMOS process

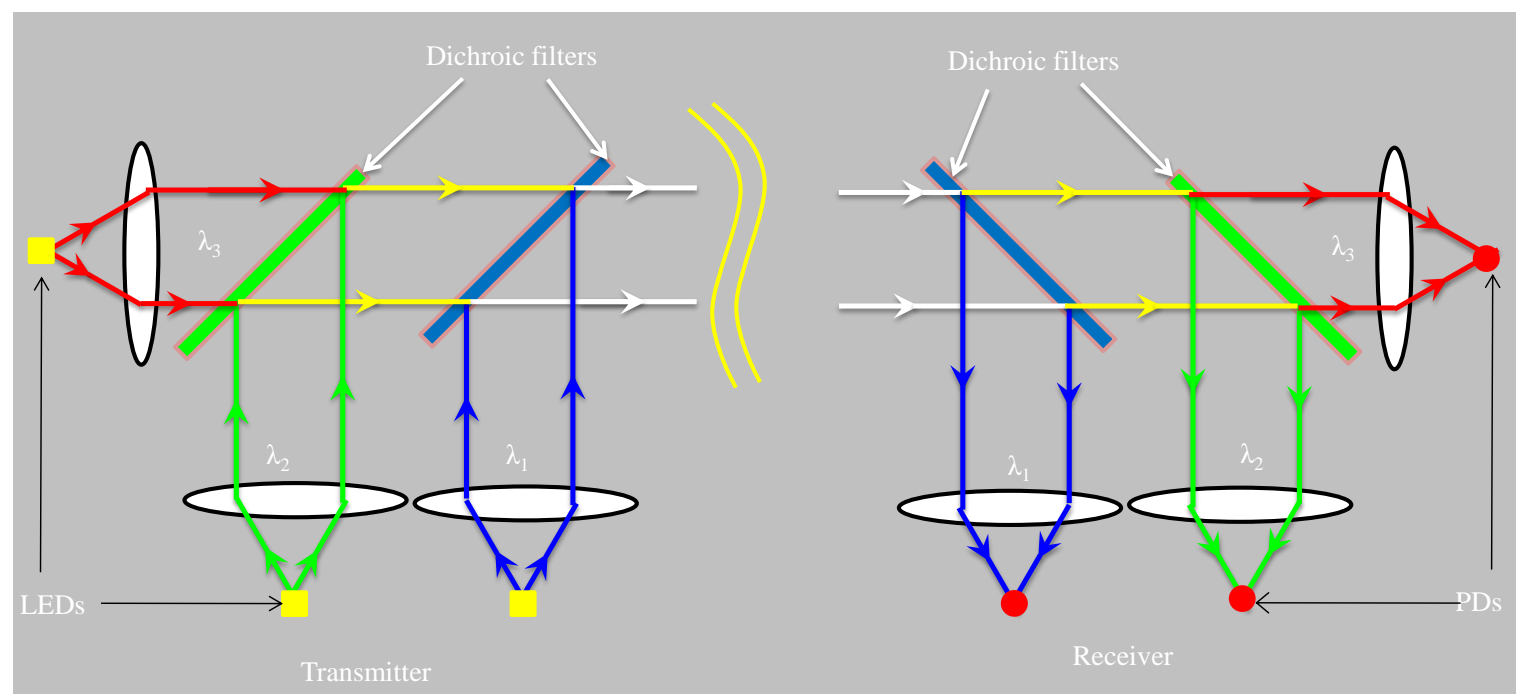

Figure 20: Schematic of a WDM-based VLC system.

\subsection{Approaches to generate white light and their data communication potential}

As described in the previous sections, there are two fundamental approaches to generate white light: by using a colour converter with a blue or ultraviolet source or by mixing the emissions from multiple monochromatic sources. The white light generated by mixing monochromatic sources enables WDM and offers high data rates, as described in section 4.5. This approach to white light generation, however, is not cost effective as there is a requirement of multiple drivers and colour balancing. The phosphor approach, on the other hand, provides a cost effective solution for the white light generation and hence is currently the dominant commercial white light source.

The data communication capacity of white light generated using the passive colour conversion tends to have lower capacity due to the slow temporal response of the colour converters. As described above, Ce:YAG phosphor is currently the most popular material to generate white light and has a limited bandwidth of around $2 \mathrm{MHz}$. An optical filter that rejects the yellowish emission from the received signal improves the system bandwidth. Using the optical filter, advanced modulation techniques and further signal processing, a Gbit/s VLC system was demonstrated using phosphor LEDs [132], [161]. The signal attenuation by a blue filter that rejects the longer wavelength is not negligible, however, and the potential benefit to achieve the higher data rate using a blue filter is questionable [211], [290], [291]. The study in [211], [290] showed that the blue filtering is not essential for advanced modulation schemes like DMT, CAP with DFEs. Nonetheless, blue filtering is a simple way to improve system capacity without increasing the computational complexity. 
To overcome the limited bandwidth of Ce:YAG, alternative materials suitable for high-speed VLC systems have been investigated. Colloidal quantum dots (CQDs) with CdSe/ZnS core-shell structure are suitable down-converting materials for white light emission from the blue and ultraviolet (UV) wavelengths [292], [293]. CQDs typically have an order of magnitude higher bandwidth than typical rare-earth-doped phosphors. Moreover, the CQD emission spectra have a narrow FWHM of $\sim 30 \mathrm{~nm}$ and the centre wavelength can be customized, thus paving the way for WDM [294]. A study carried out by Laurand et al. showed a clear 'eyeopening' (in an eye diagram) at $25 \mathrm{Mb} / \mathrm{s}$ OOK -NRZ with the wavelength-converted light [294]. Inorganic epitaxial multiple quantum well (MQW) colour converters can further improve the efficacy of white LEDs beyond 4001m/W [295]. A VLC system using hybrid LED based on II-VI CdMgZnSe MQW membrane bonded onto a blue $\mu$ LED was studied in [296]. The study illustrated an optical bandwidth of the hybrid-LED of $>50$ $\mathrm{MHz}$, significantly higher than that of phosphor based LEDs.

Among the colour conversion techniques, arguably the best potential (in terms of communications capability) is shown by organic conjugated polymers. Organic polymers can be found in organic light-emitting diodes (OLEDs), now widely use in commercial applications including smartphone displays and televisions. These materials have tunable emission from the near ultraviolet to the near infra-red, short radiative lifetime, and high photoluminescence quantum yield (PLQY), making them suitable for VLC applications. A number of such organic semiconductor colour converters covering a range of visible emission spectra were studied in [297], [298]. The bandwidths of these colour converters are 100's of MHz, significantly higher than commercial blue LEDs and hence they do not impose a bottleneck for VLC. Combining the PL from a copolymer of polypara-phenylene vinylene, named 'Super Yellow' (S-Y; PDY132, Merck KGaA) and the blue EL of a GaN $\mu \mathrm{LED}$, the authors in [26] generated white light suitable for illumination. S-Y has a bandwidth in excess of $100 \mathrm{MHz}$, which is a $>50$ fold increase in comparison to Ce:YAG (see the frequency response shown in Figure 21). A data rate of $1.68 \mathrm{~Gb} / \mathrm{s}$ was demonstrated using the white spectrum (without any optical filter at the receiver) generated using the S-Y based white LED at an illumination level of 240 lux and a PIN photodiode receiver [26]. At a similar lux level using similar receiver technology, the Ce:YAG a based white LED can only achieve a data rate of $\sim 300 \mathrm{Mb} / \mathrm{s}$ [157], clearly demonstrating the advantages of the polymer colour converter . The data rate was further improved to $2.3 \mathrm{~Gb} / \mathrm{s}$ using WDM-based on blue LEDs and green and red colourconverters [38]. These studies clearly demonstrated the potential of polymer colour converters for VLC applications.

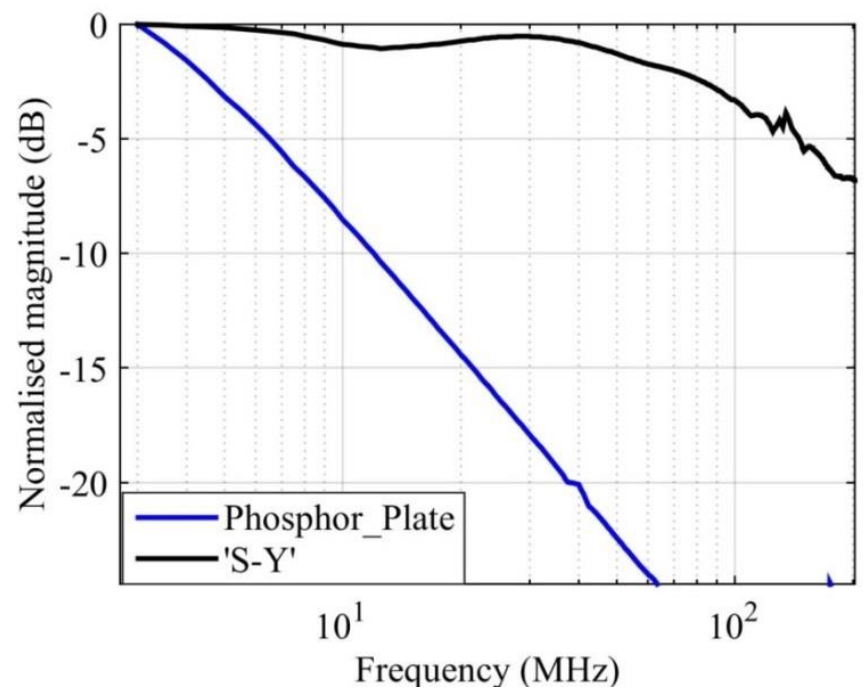

Figure 21: Frequency response of 'Ce:YAG' (phosphor plate) and 'S-Y' (polymer) colour converters.

\section{Applications of VLC}

Due to the wide availability of LEDs based lighting and signalling infrastructure, there are wide-ranging VLC applications such as a dedicated high-speed link in a room and office environments, underwater communication, vehicular communication, machine-to-machine communication. Some of the key applications that have been considered at present are discussed in this section. 


\section{a) High-speed data communication}

There are several application scenarios for indoor free space VLC where data transmission utilises SSL infrastructure, conventionally installed in ceiling fixtures. In a business or domestic environment with relatively small rooms, the blocking of signals by walls can be advantageous for security, and the links are not dependent on LOS conditions. Furthermore, VLC can avoid the need for RF emitters in sensitive environments such as hospitals and laboratories. The coverage of indoor LED transmitters is a few square metres, leading naturally to the so-called attocell concept in LiFi [4], which can support multi-user access in larger enclosed spaces such as transport terminals or supermarkets.

Guided wave VLC applications relevant to this review include the use of inexpensive plastic optical fibre (POF), for transmission over 10-100 metre distances. In building installations, POF-based VLC can effectively transmit data between rooms or apartments, and so complement more localised free space VLC links [56]. Such networks are also particularly easy to modify and extend, as splicing techniques for POF are non-demanding. POF-based VLC is also well suited to applications in automobiles and aircraft, where immunity to RF interference gives critical safety advantages and mass savings may also be important. Standards for POF-based automotive VLC, currently using red-emitting visible LEDs, have been developed for over a decade, and as of 2016 such installations can be found in over 200 car models sold by multiple manufacturers [299]. A distinct application for guided wave VLC uses planar polymer waveguides for high-density optical interconnections, notably at a board-to-board level in data centres [300]. Typical embodiments incorporate layers containing polymer waveguide arrays into multi-layer printed circuit boards also populated with metal tracks, and future developments will extend the technology to 2-dimensional waveguide arrays [301].

\section{b) Intelligent transport system (ITS)}

As with lighting infrastructure found in buildings, light sources used on street lamps and vehicle head and taillights are either already replaced or will soon be replaced by LEDs. This makes it feasible to make use of the lighting infrastructure for signalling, communication, and positioning, leading to the concept of an ITS. VLC is now considered for communication within a vehicle [302], between vehicles (i.e. vehicle-to-vehicle (V2V) communication) [303]-[308] and vehicle-to-Infrastructure (V2I) [309]-[311]. ITS systems can make use of LED arrays available within traffic lights, headlights and tail-lights as transmitters, and on-board camera systems a receivers [306], [311]. Since the application of VLC for ITS involves outdoor environment and strong ambient light, the communication channel is significantly different to the indoor environment. The performance of $\mathrm{V} 2 \mathrm{I}$ and $\mathrm{V} 2 \mathrm{~V}$ communication depends on environmental factors such as fog, rain and smoke [307]. A number of studies have been carried out to model V2V VLC channels [304], [305]. In order to provide reliable communication for a fast moving vehicle, ITS often incorporates tracking algorithms [309].

\section{c) Underwater communications}

VLC is considered as an alternative to acoustic communication for underwater communication, especially for sensor networks, high-speed communications for autonomous underwater vehicle (AUV), and diver communications [312]-[315]. RF signals have a high attenuation in water and hence are not suitable for underwater communication. Though acoustic communications are currently used for long distance underwater communication, they suffer from a limited data rate and high latency due to the low speed of sound in water. Hence, VLC could play a vital role for short range underwater communication, especially for diver communications. Underwater VLC channel suffers from high attenuation and multiple scattering. Scattering and attenuation are strongly wavelength dependent, and hence underwater communication systems use blue-green (400-550 nm) LEDs to exploit the low absorption in this wavelength region [316]-[318].

\section{d) Indoor Positioning}

The global position system (GPS) does not work well in an indoor environment. There are cases where indoor navigation is important in places like tunnels, mines, museums, supermarkets and hospitals. Using the existing indoor lighting infrastructure, VLC can be combined with indoor localisation and navigation is feasible [319], and VLC-based indoor positioning is well suited to the enclosed environments just mentioned [320]. Unlike RF based indoor positioning, VLC based positioning suffers less severely from multipath propagation and hence positioning accuracy is higher than with RF systems. Though positioning based on the received signal strength 
(RSS) (which is very popular in RF positioning) is considered by a number of researchers [321]-[323], alternative localisation algorithms based on the angle of arrival, time difference of arrival (TDOA) are also considered for higher accuracy. A detailed comparison of these location algorithms is surveyed in [324] and hence it is not repeated here.

\section{e) Other applications}

VLC has potential to provide ubiquitous networking for sensor networks [325], internet of things (IoT) and machine-to-machine (M2M) communication [79], [326]. As LEDs are widely used as a visual indicators in most modern mobile devices, home appliances, and industrial and commercial equipment it is feasible to use them in a communication transceiver and hence possible to interconnect billions of machines and objects. The Walt Disney Company has already demonstrated toy-to-toy communication using LEDs as transceiver [327] proving the potential of optical interconnects for IoT. Other researchers considered near field communication for mobile payment [328], barcode reading for smartphones [329], to name a few of many possible applications.

\section{Challenges and open issues}

\section{a) Commercialisation}

Though VLC has potential to provide secure, high-speed data communication, the market penetration is gaining momentum only lately. The survey by MarketsandMarkets predicts that the compound annual growth rate (CAGR) of VLC from 2015 to 2020 will be over 90\% [330]. A number of companies including PureLiFIi (UK), Phillips (Netherlands), Disney (Switzerland), Hua Wei Communications (UK), OLEDComm (France), AXRTEK (US), and HHI (Germany) are some of the companies that are involved in research and development of VLC technology, and now offer commercial solutions.

The commercial success of VLC largely depends on how the OWC (wireless infrared and visible communication) ecosystem will evolve in the future, and also the number of applications developed for this ecosystem. One of the key challenges for VLC is that in order to offer the best VLC service, there must be coordination of two industries, lighting and communication [331]. There has already been an effort by Philips in collaboration with French supermarket chain Carrerfour to deliver indoor localisation to the customer using lighting infrastructure and user mobile device camera [332]. VLC has also been standardised by IEEE (IEEE 802.15.7) along with other short range OWC technologies [19], [20] and is widely expected to be a part of 5G networks.

\section{b) Modulation bandwidth of LEDs}

The illumination industries still use large area blue LED and phosphor colour converters, which are suitable for mass production. However, phosphor based white LEDs have a limited bandwidth of a few MHz, making them non-ideal for high-speed communication. Though we have discussed high-bandwidth LEDs and colour converters in this paper, these are developed primarily to increase the modulation bandwidth and are still far from being adopted by illumination industries. The adaptation of these LEDs to suit illumination industries will take time and depends on the additional benefit (i.e. revenue) that can be generated by adopting the new technologies, which in turn depends on the market penetration of VLC. Though it is feasible to increase the modulation bandwidth using equalisation techniques as outlined in Section 4.3, equalisation increases system complexity (and hence cost) as well as incurring a power penalty.

\section{c) LED nonlinearity and dynamic range}

The current-power response of LEDs is non-linear and has a limited dynamic range. LED non-linearity has a significant impact on performance as non-linearity introduces amplitude and phase distortion. The impact of non-linearity is more severe in high order sub-carrier modulation like OFDM due to high PAPR. In order to minimise the impact of non-linearity, it is necessary to bias LEDs in an optimum level and also limit the peakto-peak input signal [333], [334]. The non-linearity can be mitigated by using pre-distortion [200], postdistortion [231], [232] or using non-linear equalisers [130]. 


\section{d) Mobility and coverage}

Most high-speed VLC applications consider a narrow FOV LOS link which can utilise a dense deployment of LEDs. Also, a small cell radius (less than few $\mathrm{m}^{2}$ ) offers a high spatial density, which will naturally increase the overall system capacity. However, the small cell radius creates two fundamental challenges: a) the high spatial density causes high inter-cell interference (ICI) and b) a small coverage area requires frequent hand-over for mobile terminals, increasing system complexity and overhead. The ICI can be minimised by using different wavelengths at neighbouring cells. However, illumination constraints may not always permit use of different wavelengths. Alternately, a MIMO concept where each receiver is trained to nullify the ISI can be used to solve the problem. The need for frequency hand-over can be minimised by using a wider divergence angle transmitter and a diffuse link to provide large area coverage. A diffuse link has limited channel capacity due to multipath propagation, but can be advantageous if applications do not require a dedicated high-speed link.

\section{e) Convergence/ Augmentation with Existing Systems}

It is widely accepted that the VLC will complement the existing RF network to provide dedicated high-speed data links. However, there has not been significant research work to integrate VLC with existing wired and wireless infrastructure. It is not cost effective to deploy dedicated infrastructure for VLC, and there is limited research and development work to make the current backbone network infrastructure compatible with RF and VLC systems. Considering that VLC and RF are different technologies, significant research and development activities are necessary to fully benefit from their unique advantages. This possibly requires not only development in the physical layer, but also the development of new algorithms for media access control (MAC) and higher level. Except for the FP7 project on Home Access Networks (OMEGA), which investigated the integration of power line communication (PLC) with VLC [16], there had not been extensive afford to ingrate VLC with existing communication infrastructure. As VLC systems penetrates communication markets, we can expect such issues to be addressed in the future.

\section{Conclusions}

With the evolution and rapid growth of the deployment of visible-emitting solid state lighting devices, the integration of illumination and communications can provide significant benefits as high-speed data communications can be realized using existing lighting infrastructure with minimal additional cost. Moreover, the so-called 'spectrum-crunch' in the utilization of the RF spectrum appears unavoidable due to the limited available RF spectral range and the exponential increase of the network traffic. In order to leverage the potential of communications technologies, it is essential that in the future a number of complementary technologies coexist servicing the specific areas to which each technology is most suited. VLC is one of the key technologies among those having the high potential of providing $\mathrm{Gb} / \mathrm{s}$ data rates using the $\sim 300 \mathrm{THz}$ of the unregulated visible spectrum. This paper presents a survey of GaN-based VLC systems with focus on a number of key developments over the last two decades that have enabled practical demonstration of $\mathrm{Gb} / \mathrm{s}$ data rates.

First envisioned in 1979 by Gfeller and Bapst, VLC has advanced significantly by achieving two orders of magnitude higher data rates within less than a decade and now reaching data rate beyond $10 \mathrm{~Gb} / \mathrm{s}$. As the research pushes towards high data rate practical systems, a number of new technologies have emerged. Early VLC demonstrations used commercial white LEDs. Though a few hundreds $\mathrm{Mb} / \mathrm{s}$ up to $\mathrm{Gb} / \mathrm{s}$ data rates were achieved by using these LEDs with equalization and advanced modulation schemes, it was realized that the phosphor used in the commercial white LEDs provides severe challenges in producing multi-Gb/s systems. Hence, high bandwidth micro-LEDs and alternative approaches in white light generation using RGB LEDs, organic colour converters, and CQDs have been introduced. Furthermore, in recent years, there is an increasing emphasis towards spatial multiplexing and wavelength division multiplexing for multi-Gb/s system. Some of these technologies are still in the embryonic stage, but have already proved their fundamental potential. Hence, it is expected that further research and development will offer even higher data rate VLC systems that are energy efficient, robust and resilient. To take full advantage of the benefits of high-speed VLC links, there is also a growing need to develop suitable protocols for the higher network layers. 


\section{Acknowledgement}

The authors gratefully acknowledge support by the UK Engineering and Physical Sciences Research Council (EPSRC) under grant EP/K00042X/1. We also gratefully acknowledge the contributions to this work of all the partners in UP-VLC.

\section{Reference}

[1] "Cisco Visual Networking Index: Forecast and Methodology, 2014-2019," 2016. [Online]. Available: http://www.cisco.com/c/en/us/solutions/collateral/service-provider/ip-ngn-ip-next-generation-network/white_paper_c11481360.pdf.

[2] C. X. Wang, F. Haider, X. Gao, X. H. You, Y. Yang, D. Yuan, H. M. Aggoune, H. Haas, S. Fletcher, and E. Hepsaydir, “Cellular architecture and key technologies for 5G wireless communication networks," IEEE Commun. Mag., vol. 52, no. 2, pp. 122-130, 2014.

[3] M. Ayyash, H. Elgala, A. Khreishah, V. Jungnickel, T. Little, S. Shao, M. Rahaim, D. Schulz, J. Hilt, and R. Freund, “Coexistence of WiFi and LiFi towards 5G: Concepts, Opportunities, and Challenges," IEEE Commun. Mag., vol. 54, no. 2, pp. 64-71, 2016.

[4] H. Haas, L. Yin, Y. Wang, and C. Chen, "What is LiFi?," J. Light. Technol., vol. 36, no. 6, pp. 1533 - $1544,2016$.

[5] S. Wu, H. Wang, and C. Youn, "Visible light communications for 5G wireless networking systems: From fixed to mobile communications," IEEE Netw., vol. 28, no. 6, pp. $41-45,2014$

[6] S. Zvanovec, P. Chvojka, P. Haigh, and Z. Ghassemlooy, "Visible Light Communications towards 5G," Radioengineering, vol. 24, no. 1, pp. 1-9, 2015.

[7] U. S. D. of Energy, "Energy savings forecast of solid-state lighting in general illumination applications," 2014

[8] R. Baleja, J. Sumpich, P. Bos, B. Helstynova, K. Sokansky, and T. Novak, "Comparison of LED properties, compact fluorescent bulbs and bulbs in residential areas," in 16th International Scientific Conference on Electric Power Engineering (EPE), 2015, 2015, pp. 566-571.

[9] R. Haitz and J. Y. Tsao, "Solid-state lighting: 'The case' 10 years after and future prospects," Phys. Status Solidi Appl. Mater. Sci., vol. 208, no. 1, pp. 17-29, 2011.

[10] M. H. Crawford, "LEDs for solid-state lighting: Performance challenges and recent advances," IEEE J. Sel. Top. Quantum Electron., vol. 15, no. 4, pp. 1028-1040, 2009.

[11] D. O’Brien, G. Parry, and P. Stavrinou, "Optical hotspots speed up wireless communication," Nat. Photonics, vol. 1, no. 5, pp. 245-247, 2007.

[12] G. Pang and T. Kwan, "LED traffic light as a communications device," in IEEE/IEEJ/JSAI International Conference on Intelligent Transportation Systems, 1999, pp. 788-793.

[13] Y. Tanaka, S. Haruyama, and M. Nakagawa, "Wireless optical transmissions with white colored LED for wireless home links," in The 11th IEEE International Symposium on Personal, Indoor and Mobile Radio Communications (PIMRC) 2000., 2000, vol. 2, pp. 1325-1329.

[14] C. G. Lee, "Visible Light Communication," in Short-Range Wireless Communications: Emerging Technologies and Applications, vol. 11, no. 01, R. Kraemer and M. Katz, Eds. Wiley Publishing, 2015, pp. 327-338.

"visible light communication associations (VLCA)." [Online]. Available: http://vlca.net.

[16] "hOME Gigabit Access project (OMEGA)." [Online]. Available: http://www.ict-omega.eu.

[16] "hOME Gigabit Access project (OMEGA)." [Online]. Available: http://www.ict-omega.eu.

[18] H. Chun, S. Rajbhandari, G. Faulkner, D. Tsonev, E. Xie, J. J. D. McKendry, E. Gu, M. D. Dawson, D. O’Brien, and H. Haas, "LED based wavelength division multiplexed $10 \mathrm{~Gb} / \mathrm{s}$ visible light communications," J. Light. Technol., vol. 34, no. 13, pp. 3047 $-3052,2016$.

[19] S. Rajagopal, R. D. Roberts, and L. Sang-Kyu, "IEEE 802.15.7 visible light communication: modulation schemes and dimming support," IEEE Commun. Mag., vol. 50, no. 3, pp. 72-82, 2012

[20] A. C. Boucouvalas, P. Chatzimisios, Z. Ghassemlooy, M. Uysal, and K. Yiannopoulos, "Standards for indoor optical wireless communications," IEEE Commun. Mag., vol. 53, no. 3, pp. 24-31, 2015.

[21] "IEEE 802.15.7r." [Online]. Available: http://www.ieee802.org/15/pub/IEEE 802 15 WPAN 157 Revision1 Task Group.htm. [Accessed: 19-Apr-2016].

[22] H. Le Minh, D. O. Brien, G. Faulkner, L. Zeng, K. Lee, D. Jung, and Y. Oh, "80 Mbit/s visible light communications using preequalized white LED," in 34th European Conference on Optical Communication, 2008. ECOC 2008., 2008, vol. 5, no. September, pp. $1-2$.

[23] J. J. D. McKendry, R. P. Green, A. E. Kelly, G. Zheng, B. Guilhabert, D. Massoubre, E. Gu, and M. D. Dawson, "High-speed visible light communications using individual pixels in a micro light-emitting diode array," IEEE Photonics Technol. Lett., vol. 22, no. 18 , pp. $1346-1348,2010$.

[24] R. X. G. Ferreira, E. Xie, J. J. D. McKendry, S. Rajbhandari, H. Chun, G. Faulkner, S. Watson, A. E. Kelly, E. Gu, R. V Penty, I. H. White, D. C. O’Brien, and M. D. Dawson, "High bandwidth GaN-based micro-LEDs for multi-Gbps visible light communications," IEEE Photonics Technol. Lett., vol. 28, no. 19, pp. 2023-2026, 2016.

[25] D. Tsonev, H. Chun, S. Rajbhandari, J. McKendry, S. Videv, E. Gu, M. Haji, S. Watson, A. Kelly, G. Faulkner, M. Dawson, H. Haas, and D. O'Brien, "A 3-Gb/s single-LED OFDM-based wireless VLC link using a Gallium Nitride $\mu$ LED," IEEE Photonics Technol. Lett., vol. 26, no. 7, pp. 637-640, 2014.

[26] H. Chun, P. Manousiadis, S. Rajbhandari, D. A. Vithanage, G. Faulkner, D. Tsonev, J. J. D. McKendry, S. Videv, X. Enyuan, G. Erdan, M. D. Dawson, H. Haas, G. A. Turnbull, I. D. W. Samuel, and D. C. O’Brien, "Visible light communication using a blue GaN $\mu$ LED and fluorescent polymer color converter," IEEE Photonics Technol. Lett., vol. 26, no. 20, pp. $2035-2038,2014$.

[27] L. Chien-Lan, H. Chong-Lung, C. Yung-Fu, W. Chi-Hung, and W. Meng-Chyi, "High-speed light-emitting diodes emitting at 500 nm with 463-MHz modulation bandwidth," IEEE Electron Device Lett., vol. 35, no. 5, pp. 563-565, 2014.

[28] J. W. Shi, C. W. L. Lin, W. Chen, J. E. Bowers, J. K. Sheu, C. W. L. Lin, Y. L. Li, J. Vinogradov, O. Ziemann, L. Che-Wei, C. Wei, J. E. Bowers, J. K. Sheu, L. Ching-Liang, L. Yun-Li, J. Vinogradov, and O. Ziemann, "Very high-speed GaN-based cyan light emitting diode on patterned sapphire substrate for 1 Gbps plastic optical fiber communication," in Optical Fiber Communication Conference and Exposition (OFC/NFOEC), 2012 and the National Fiber Optic Engineers Conference, 2012, pp. $1-3$.

[29] T. Dongdong, W. Mingyang, L. Lilin, and W. Gang, "Size- and current-density-controlled tunable wavelength in GaN-based LEDs for potential dense wavelength-division multiplexing application," IEEE Wirel. Commun., vol. 22, no. 2, pp. 74-79, 2015. 
[30] G. Cossu, W. Ali, R. Corsini, and E. Ciaramella, "Gigabit-class optical wireless communication system at indoor distances (1.5 $\div$ 4 m)," Opt. Express, vol. 23, no. 12, pp. 15700-15705, 2015.

[31] D. Tsonev, S. Videv, and H. Haas, “Towards a $100 \mathrm{~Gb} / \mathrm{s}$ visible light wireless access network," Opt. Express, vol. 23, no. 2, pp. 1627-1637, 2015.

[32] S. Hranilovic and F. R. Kschischang, "A pixelated MIMO wireless optical communication system,” IEEE J. Sel. Top. Quantum Electron., vol. 12, no. 4, pp. 859-874, 2006.

[33] S. Rajbhandari, A. V. N. Jalajakumari, H. Chun, G. Faulkner, K. Cameron, R. Henderson, D. Tsonev, H. Haas, E. Xie, J. J. D. McKendry, J. Herrnsdorf, R. Ferreira, E. Gu, M. D. Dawson, and D. O’Brien, "A Multi-Gigabit/sec Integrated Multiple-Input Multiple-Output VLC Demonstrator," J. Light. Technol. [Unpublished], 2016.

[34] Y. Wang, L. Tao, X. Huang, J. Shi, and N. Chi, "8-Gb/s RGBY LED-Based WDM VLC System Employing High-Order CAP Modulation and Hybrid Post Equalizer," IEEE Photonics J., vol. 7, no. 6, pp. 1-7, 2015.

[35] R. Mesleh, H. Elgala, and H. Haas, "Optical Spatial Modulation,” J. Opt. Commun. Netw., vol. 3, no. 3, p. 234 , Feb. 2011.

[36] R. Singh, T. O'Farrell, and J. David, "An Enhanced Colour Shift Keying Modulation Scheme for High Speed Wireless Visible Light Communications,” J. Light. Technol., vol. 32, no. 14, pp. 2582 - 2592, 2014.

[37] S. Dimitrov, S. Member, and H. Haas, "Information Rate of OFDM-Based Optical Wireless Communication Systems With Nonlinear Distortion,” J. Light. Technol., vol. 31, no. 6, pp. 918-929, 2013.

[38] P. Manousiadis, C. Hyunchae, S. Rajbhandari, R. Mulyawan, D. A. Vithanage, G. Faulkner, D. Tsonev, J. J. D. McKendry, M. Ijaz, X. Enyuan, E. Gu, M. D. Dawson, H. Haas, G. A. Turnbull, I. D. W. Samuel, and D. O’Brien, "Demonstration of 2.3 Gb/s RGB white-light VLC using polymer based colour-converters and GaN micro-LEDs," in Summer Topicals Meeting Series (SUM), 2015, 2015, pp. 222-223.

[39] D. A. Steigerwald, J. C. Bhat, D. Collins, R. M. Fletcher, M. O. Holcomb, M. J. Ludowise, P. S. Martin, and S. L. Rudaz, "Illumination with solid state lighting Technology," IEEE J. Sel. Top. Quantum Electron., vol. 8, no. 2, pp. 310-320, 2002.

[40] M. S. Shur and A. Žukauskas, "Solid-state lighting: Toward superior illumination," Proc. IEEE, vol. 93, no. 10, pp. 1691-1703, 2005.

[41] J. J. Wierer, J. Y. Tsao, and D. S. Sizov, "Comparison between blue lasers and light-emitting diodes for future solid-state lighting," Laser Photonics Rev., vol. 7, no. 6, pp. 963-993, 2013.

[42] H. Chun, S. Rajbhandari, D. Tsonev, G. Faulkner, H. Haas, and D. O'Brien, "Visible light communication using laser diode based remote phosphor technique," in Communication Workshop (ICCW), 2015 IEEE International Conference on, 2015, pp. 13921397.

[43] Y.-C. Chi, D.-H. Hsieh, C.-Y. Lin, H.-Y. Chen, C.-Y. Huang, J.-H. He, B. Ooi, S. P. DenBaars, H.-C. Kuo, and G.-R. Lin, "Phosphorous diffuser diverged blue laser diode for indoor lighting and communication," Sci. Rep., vol. 5, no. 18690, pp. 1-9, 2015.

[44] I. A. Barlow, T. Kreouzis, and D. G. Lidzey, "High-speed electroluminescence modulation of a conjugated-polymer light emitting diode," Appl. Phys. Lett., vol. 94, no. 24, p. 243301, 2009.

[45] P. A. Haigh, Z. Ghassemlooy, S. Rajbhandari, and I. Papakonstantinou, "Visible light communications using organic light emitting diodes," IEEE Commun. Mag., vol. 51, no. 8, pp. 148-154, 2013.

[46] H. Chun, C. J. Chiang, A. Monkman, and D. Obrien, "A study of illumination and communication using organic light emitting diodes," J. Light. Technol., vol. 31, no. 22, pp. 3511-3517, 2013.

[47] Y. Qiu, H.-H. Chen, and W.-X. Meng, "Channel modeling for visible light communications-a survey," Wirel. Commun. Mob. Comput., vol. 16, no. 14, pp. 2016-2034, Feb. 2016.

[48] J. M. Kahn and J. R. Barry, "Wireless infrared communications," Proc. IEEE, vol. 85, no. 2, pp. $265-298,1997$.

[49] Z. Ghassemlooy, W. O. Popoola, and S. Rajbhandari, Optical wireless communications - System and channel modelling with Matlab, 1st ed. CRC Press, 2012.

[50] L. Kwonhyung, P. Hyuncheol, and J. R. Barry, "Indoor channel characteristics for visible light communications," IEEE Commun. Lett., vol. 15, no. 2, pp. 217-219.

[51] A. Sivabalan and J. John, "Modeling and simulation of indoor optical wireless channels: a review," in TENCON 2003. Conference on Convergent Technologies for Asia-Pacific Region, 2003, vol. 3, pp. 1082-1085.

[52] D. Wu, Z. Ghassemlooy, H. Le Minh, S. Rajbhandari, and A. C. Boucouvalas, "Improvement of the Transmission bandwidth for indoor optical wireless communication systems using a diffused Gaussian beam," Commun. Lett. IEEE, vol. 16, no. 8, pp. 1316$1319,2012$.

[53] J. B. Carruthers, S. M. Caroll, and P. Kannan, "Propagation modelling for indoor optical wireless communications using fast multi-receiver channel estimation," IEE Proceedings-Optoelectron., vol. 150, no. 5, pp. 473-481, 2003.

[54] K. Cui, G. Chen, Z. Xu, and R. D. Roberts, "Traffic light to vehicle visible light communication channel characterization," Appl. Opt., vol. 51, no. 27, pp. 6594-6605, 2012.

[55] X. Li, N. Bamiedakis, J. L. Wei, J. J. D. McKendry, E. Xie, R. Ferreira, E. Gu, M. D. Dawson, R. V Penty, and I. H. White, " $\mu$ LED-based single-wavelength bi-directional POF link with $10 \mathrm{~Gb} / \mathrm{s}$ aggregate data rate," J. Light. Technol., vol. PP, no. 99, p. $1,2015$.

[56] Y. Dong and K. W. Martin, "Gigabit communications over plastic optical fiber opportunities and challenges for the home network," IEEE Solid State Circuits Mag., vol. 3, no. 1, pp. 60-69, 2011.

[57] J. Vinogradov, R. Kruglov, C. Kai-Lun, S. Jin-Wei, M. Bloos, S. Loquai, and O. Ziemann, "GaN light-emitting diodes for up to 5.5-Gb/s short-reach data transmission over SI-POF," IEEE Photonics Technol. Lett., vol. 26, no. 24, pp. $2473-2475,2014$.

[58] P. J. Pinzón, I. P. Garcilópez, and C. Vázquez, "Efficient multiplexer/demultiplexer for visible WDM transmission over SI-POF technology," J. Light. Technol., vol. 33, no. 17, pp. 3711-3718, 2015.

[59] N. T. Le, M. S. Ifthekhar, Y. M. Jang, and N. Saha, "Survey on optical camera communications: challenges and opportunities," IET Optoelectron., vol. 9, no. 5, pp. 172-183, 2015.

[60] S. D. Perli, N. Ahmed, and D. Katabi, "PixNet: interference-free wireless links using LCD-camera pairs," Proceedings of the sixteenth annual international conference on Mobile computing and networking. ACM, Chicago, Illinois, USA, pp. 137-148, 2010.

[61] T. Yamazato, I. Takai, H. Okada, T. Fujii, T. Yendo, S. Arai, M. Andoh, T. Harada, K. Yasutomi, K. Kagawa, and S. Kawahito, "Image-sensor-based visible light communication for automotive applications," IEEE Commun. Mag., vol. 52, no. 7, pp. 88-97, 2014.

[62] C. Danakis, M. Afgani, G. Povey, I. Underwood, and H. Haas, "Using a CMOS camera sensor for visible light communication," in 2012 IEEE Globecom Workshops, GC Wkshps 2012, 2012, pp. 1244-1248.

[63] T.-H. Do and M. Yoo, "Performance analysis of visible light communication using CMOS sensors," Sensors, vol. 16, no. 3, p. 309, 2016. 
[64] P. Luo, M. Zhang, Z. Ghassemlooy, H. Le Minh, H.-M. Tsai, X. Tang, and D. Han, "Experimental demonstration of a 1024-QAM optical camera communication system," IEEE Photonics Technol. Lett., vol. 28, no. 2, pp. 139-142, Jan. 2016.

[65] O. Kharraz and D. Forsyth, "Performance comparisons between PIN and APD photodetectors for use in optical communication systems," Optik (Stuttg)., vol. 124, no. 13, pp. 1493-1498, 2013.

[66] H. Nie, "High performance, low-cost PIN, APD receivers in fiber optical networks and FTTx applications," in 14th Annual International Conference on Wireless and Optical Communications, 2005. WOCC 2005, pp. 94-94.

[67] D. Chitnis, L. Zhang, H. Chun, S. Rajbhandari, G. Faulkner, D. O'Brien, and S. Collins, "A $200 \mathrm{Mb} / \mathrm{s}$ VLC demonstration with a SPAD based receiver," in 2015 IEEE Summer Topicals Meeting Series (SUM), 2015, pp. 226-227.

[68] I. Alsolami, D. Chitnis, D. C. O'Brien, and S. Collins, "Broadcasting over photon-counting channels via multiresolution PPM: Implementation and experimental results," IEEE Commun. Lett., vol. 16, no. 12, pp. 2072-2074, 2012.

[69] M. Safari, R. Henderson, and H. Haas, "Optical OFDM with single-photon avalanche diode," IEEE Photonics Technol. Lett., vol. 27, no. 9, pp. 943-946, May 2015.

[70] O. Almer, D. Tsonev, N. A. W. Dutton, T. Al Abbas, S. Videv, S. Gnecchi, H. Haas, and R. K. Henderson, "A SPAD-based visible light communications receiver employing higher order modulation," in 2015 IEEE Global Communications Conference (GLOBECOM), 2015, pp. 1-6.

[71] A. Kerans, D. Vo, P. Conder, and S. Krusevac, "Pricing of spectrum based on physical criteria," in 2011 IEEE International Symposium on Dynamic Spectrum Access Networks, DySPAN 2011, 2011, pp. 223-230.

[72] "Price hike for UK mobile spectrum." [Online]. Available: http://www.bbc.co.uk/news/technology-34346822. [Accessed: 26May-2016].

[73] "Radio Spectrum Allocation." [Online]. Available: https://www.fcc.gov/engineering-technology/policy-and-rulesdivision/general/radio-spectrum-allocation. [Accessed: 20-May-2016].

[74] P. Goud, C. Schlegel, W. A. Krzymien, and R. Hang, "Multiple-antenna communication systems: an emerging technology," Can. J. Electr. Comput. Eng., vol. 29, no. 1/2, pp. 51-59, Jan. 2004.

[75] S.-M. Kim and J.-B. Jeon, "Experimental Demonstration of $4 \times 4$ MIMO Wireless Visible Light Communication Using a Commercial CCD Image Sensor,” J. Inf. Commun. Converg. Eng., vol. 10, no. 3, pp. 220-224, 2012.

[76] H. Waves, "What you see is what you send," Eng. Technol., vol. 3, no. 19, pp. 66-69, 2008.

[77] G. Corbellini, K. Akșit, S. Schmid, S. Mangold, and T. R. Gross, "Connecting networks of toys and smartphones with visible light communication," IEEE Commun. Mag., vol. 52, no. 7, pp. 72-78, 2014.

[78] S. Schmid, J. Ziegler, T. R. Gross, M. Hitz, A. Psarra, G. Corbellini, and S. Mangold, "(In)visible light communication: Combining illumination and communication," in ACM SIGGRAPH 2014 Emerging Technologies, 2014, pp. 2012-2013.

[79] Z. Tian, K. Wright, and X. Zhou, "Lighting up the internet of things with DarkVLC," in Proceedings of the 17th International Workshop on Mobile Computing Systems and Applications, 2016, pp. 33-38.

[80] C. A. Hurni, A. David, M. J. Cich, R. I. Aldaz, B. Ellis, K. Huang, A. Tyagi, R. A. Delille, M. D. Craven, F. M. Steranka, and M. R. Krames, "Bulk GaN flip-chip violet light-emitting diodes with optimized efficiency for high-power operation," Appl. Phys. Lett., vol. 106, no. 3, 2015.

[81] I. M. Watson, "Metal organic vapour phase epitaxy of AlN, GaN, InN and their alloys: A key chemical technology for advanced device applications," Coord. Chem. Rev., vol. 257, no. 13-14, pp. 2120-2141, 2013.

[82] J. Piprek, "Efficiency droop in nitride-based light-emitting diodes," Phys. Status Solidi, vol. 207, no. 10, pp. 2217-2225, Oct. 2010.

[83] H. Le Minh, D. O’Brien, G. Faulkner, Z. Lubin, L. Kyungwoo, J. Daekwang, O. Yunje, and W. Eun Tae, "100-Mb/s NRZ visible light communications using a postequalized white LED,” IEEE Photonics Technol. Lett., vol. 21, no. 15, pp. 1063-1065, 2009.

[84] W. Yang, S. Zhang, J. J. D. McKendry, J. Herrnsdorf, P. Tian, Z. Gong, Q. Ji, I. M. Watson, E. Gu, M. D. Dawson, L. Feng, C. Wang, and X. Hu, "Size-dependent capacitance study on InGaN-based micro-light-emitting diodes," J. Appl. Phys., vol. 116, no. 4, p. 044512, 2014

[85] D. Delbeke, R. Bockstaele, P. Bienstman, R. Baets, and H. Benisty, "High-efficiency semiconductor resonant-cavity light-emitting diodes : A review," IEEE J. Sel. Top. Quantum Electron., vol. 8, no. 2, pp. 189-206, 2002.

[86] C. L. Tsai and Z. F. Xu, "Line-of-Sight Visible Light Communications With InGaN-Based Resonant Cavity LEDs," Photonics Technol. Lett., vol. 25, no. 18, pp. 1793-1796, 2013.

[87] M. Guina, S. Orsila, M. Dumitrescu, M. Saarinen, P. Sipilä, V. Vilokkinen, B. Roycroft, P. Uusimaa, M. Toivonen, and M. Pessa, "Light-emitting diode emitting at $650 \mathrm{~nm}$ with 200-MHz small-signal modulation bandwidth," IEEE Photonics Technol. Lett., vol. 12, no. 7, pp. 786-788, 2000.

[88] M. Pessa, M. Guina, M. Dumitrescu, I. Hirvonen, M. Saarinen, L. Toikkanen, and N. Xiang, "Resonant cavity light emitting diode for a polymer optical fibre system," Semicond. Sci. Technol., vol. 17, pp. R1-R9, 2002.

[89] A. J. Shaw, A. L. Bradley, J. F. Donegan, and J. G. Lunney, "GaN resonant cavity light-emitting diodes for plastic optical fiber applications," IEEE Photonics Technol. Lett., vol. 16, no. 9, pp. 2006-2008, Sep. 2004.

[90] J. F. Carlin, C. Zellweger, J. Dorsaz, S. Nicolay, G. Christmann, E. Feltin, R. Butté, and N. Grandjean, "Progresses in III-nitride distributed Bragg reflectors and microcavities using AllnN/GaN materials," Phys. Status Solidi Basic Res., vol. 242, no. 11, pp. 2326-2344, 2005.

[91] M. Akhter, P. P. Maaskant, B. Roycroft, B. Corbett, P. de Mierry, B. Beaumont, and K. Panzer, " 200 Mbit/s data transmission through 100m of plastic optical fibre with nitride LEDs," Electron. Lett., vol. 38, no. 23, pp. 1457-1458, 2002.

[92] O. Ziemann, J. Krauser, P. E. Zamzow, and W. Daum, POF Handbook, Second. Springer, Berlin, 2008.

[93] F. Scholz, "Semipolar GaN grown on foreign substrates: a review," Semicond. Sci. Technol., vol. 27, no. 2, p. 024002, Feb. 2012.

[94] H. Masui, S. Nakamura, S. P. DenBaars, and U. K. Mishra, "Nonpolar and Semipolar III-Nitride light-emitting diodes: Achievements and challenges," IEEE Trans. Electron Devices, vol. 57, no. 1, pp. 88-100, 2010.

[95] Y. Jho, J. Yahng, E. Oh, and D. Kim, "Field-dependent carrier decay dynamics in strained InxGa1-xN/GaN quantum wells," Phys. Rev. B, vol. 66, no. July, pp. 1-11, 2002.

[96] Z. H. Zhang, W. Liu, Z. G. Ju, S. T. Tan, Y. Ji, Z. Kyaw, X. L. Zhang, L. C. Wang, X. W. Sun, and H. V Demir, "InGaN/GaN multiple-quantum-well light-emitting diodes with a grading $\mathrm{InN}$ composition suppressing the Auger recombination," Appl. Phys. Lett., vol. 105, no. 3, p. 5, 2014.

[97] P. Waltereit, O. Brandt, A. Trampert, H. T. Grahn, J. Menniger, M. Ramsteiner, M. Reiche, and K. H. Ploog, "Nitride semiconductors free of electrostatic fields for efficient white light-emitting diodes," Nature, vol. 406, pp. 865-868, 2000

[98] M. Funato and Y. Kawakami, "Excitonic properties of polar, semipolar, and nonpolar InGaN/GaN strained quantum wells with potential fluctuations," J. Appl. Phys., vol. 103, no. 9, p. 093501, 2008.

[99] G. A. Garrett, H. E. Shen, M. Wraback, A. Tyagi, M. C. Schmidt, J. S. Speck, S. P. DenBaars, S. Nakamaura, and S. Nakamura, "Comparison of time-resolved photoluminescence from InGaN single quantum wells grown on nonpolar and semipolar bulk GaN 
substrates," Phys. Status Solidi, vol. 6, no. S2, pp. S800-S803, Jun. 2009.

[100] T. Jung, L. K. Lee, and P.-C. C. Ku, "Novel epitaxial nanostructures for the improvement of InGaN LEDs efficiency," IEEE J. Sel. Top. Quantum Electron., vol. 15, no. 4, pp. 1073-1079, 2009.

[101] H. Yang, L. X. Zheng, J. B. Li, X. J. Wang, D. P. Xu, Y. T. Wang, X. W. Hu, and P. D. Han, "Cubic-phase GaN light-emitting diodes," Appl. Phys. Lett., vol. 74, no. 17, pp. 2498-2500, 1999.

[102] H. F. Liu, H. Chen, Z. Q. Li, L. Wan, Q. Huang, J. M. Zhou, N. Yang, K. Tao, Y. J. Han, and Y. Luo, "MBE growth and Raman studies of cubic and hexagonal GaN films on (001)-oriented GaAs substrates," J. Cryst. Growth, vol. 218, no. 2-4, pp. 191-196, 2000.

[103] S. N. Waheeda, N. Zainal, Z. Hassan, S. V Novikov, A. V Akimov, and A. J. Kent, "Low fraction of hexagonal inclusions in thick and bulk cubic GaN layers," Appl. Surf. Sci., vol. 317, pp. 1010-1014, 2014.

[104] S. F. Chichibu, T. Onuma, T. Aoyama, K. Nakajima, P. Ahmet, T. Chikyow, T. Sota, S. P. DenBaars, S. Nakamura, T. Kitamura, Y. Ishida, and H. Okumura, "Recombination dynamics of localized excitons in cubic InxGa1-xN/GaN multiple quantum wells grown by radio frequency molecular beam epitaxy on 3C-SiC substrate," J. Vac. Sci. Technol. B, vol. 21, no. 4, pp. 1856-1862, 2003.

[105] C. J. M. Stark, T. Detchprohm, S. C. Lee, Y. B. Jiang, S. R. J. Brueck, and C. Wetzel, "Green cubic GaInN/GaN light-emitting diode on microstructured silicon (100)," Appl. Phys. Lett., vol. 103, no. 23, 2013.

[106] J.-W. W. Shi, J. K. K. Sheu, C.-H. H. Chen, G.-R. R. Lin, and W.-C. C. Lai, "High-speed GaN-based green light-emitting diodes with partially n-doped active layers and current-confined apertures," IEEE Electron Device Lett., vol. 29, no. 2, pp. 158-160, Feb. 2008.

[107] J.-W. W. Shi, H.-Y. Y. Huang, J. K. K. Sheu, C.-H. H. Chen, Y.-S. S. Wu, and W.-C. C. Lai, "The improvement in modulation speed of GaN-based Green light-emitting diode (LED) by use of n-type barrier doping for plastic optical fiber (POF) communication," IEEE Photonics Technol. Lett., vol. 18, no. 15, pp. 1636-1638, Aug. 2006.

[108] H. Li, P. Li, J. Kang, Z. Li, Y. Zhang, Z. Li, J. Li, X. Yi, J. Li, and G. Wang, "Quantum efficiency enhancement of 530nm InGaN green light-emitting diodes with shallow quantum well," Appl. Phys. Express, vol. 6, no. 5, pp. 1-4, 2013.

[109] S.-J. J. Lee, C.-Y. Y. Cho, S.-H. H. Hong, S.-H. H. Han, S. Yoon, S.-T. T. Kim, and S.-J. J. Park, "Enhanced optical power of inGaN/GaN light-emitting diode by ALGaN interlayer and electron blocking layer," IEEE Photonics Technol. Lett., vol. 24, no. 22, pp. 1991-1994, Nov. 2012.

[110] J.-W. Shi, K.-L. Chi, J.-M. Wun, J. Bowers, Y.-H. Shih, and J.-K. Sheu, "III-Nitride Based Cyan Light-Emitting Diodes with GHz Bandwidth for High-Speed Visible Light Communication,” IEEE Electron Device Lett., pp. 1-1, 2016.

[111] F. Qian, S. Gradečak, Y. Li, C. Y. Wen, and C. M. Lieber, "Core/multishell nanowire heterostructures as multicolor, highefficiency light-emitting diodes," Nano Lett., vol. 5, no. 11, pp. 2287-2291, 2005.

[112] S. F. Li and A. Waag, "GaN based nanorods for solid state lighting," J. Appl. Phys., vol. 111, no. 7, p. $071101,2012$.

[113] R. Koester, D. Sager, W.-A. Quitsch, O. Pfingsten, A. Poloczek, S. Blumenthal, G. Keller, W. Prost, G. Bacher, and F.-J. Tegude, "High-speed GaN/GaInN nanowire array light-emitting diode on silicon(111)," Nano Lett., vol. 15, no. 4, pp. 2318-2323, 2015.

[114] J. J. D. McKendry, D. Massoubre, S. Zhang, B. R. Rae, R. P. Green, E. Gu, R. K. Henderson, A. E. Kelly, and M. D. Dawson, "Visible-light communications using a CMOS-controlled micro-light- emitting-diode array," J. Light. Technol., vol. 30, no. 1, pp. 61-67, 2012.

[115] R. P. Green, J. J. D. McKendry, D. Massoubre, E. Gu, M. D. Dawson, and A. E. Kelly, "Modulation bandwidth studies of recombination processes in blue and green InGaN quantum well micro-light-emitting diodes," Appl. Phys. Lett., vol. 102, no. 9, 2013.

[116] C. L. Liao, Y. F. Chang, C. L. Ho, and M. C. Wu, "High-speed GaN-based blue light-emitting diodes with gallium-doped ZnO current spreading layer," IEEE Electron Device Lett., vol. 34, no. 5, pp. 611-613, May 2013.

[117] P. P. Maaskant, H. Shams, M. Akhter, W. Henry, M. J. Kappers, D. Zhu, C. J. Humphreys, and B. Corbett, "High-speed substrateemitting micro-light-emitting diodes for applications requiring high radiance," Appl. Phys. Express, vol. 6, no. 2, p. 022102, Jan. 2013.

[118] J. M. Wun, C. W. Lin, W. Chen, J. K. Sheu, C. L. Lin, Y. L. Li, J. E. Bowers, J.-W. Shi, J. Vinogradov, R. Kruglov, and O. Ziemann, "GaN-based miniaturized cyan light-emitting diodes on a patterned sapphire substrate with improved fiber coupling for very high-speed plastic optical fiber communication," IEEE Photonics J., vol. 4, no. 5, pp. 1520-1529, 2012.

[119] K. Okamoto, I. Niki, A. Scherer, Y. Narukawa, T. Mukai, and Y. Kawakami, "Surface plasmon enhanced spontaneous emission rate of InGaNGaN quantum wells probed by time-resolved photoluminescence spectroscopy," Appl. Phys. Lett., vol. 87, no. 7, 2005.

[120] X. Gu, T. Qiu, W. Zhang, and P. K. Chu, "Light-emitting diodes enhanced by localized surface plasmon resonance.," Nanoscale Res. Lett., vol. 6, no. 1, p. 199, 2011.

[121] S.-C. Zhu, Z.-G. Yu, L.-X. Zhao, J.-X. Wang, and J.-M. Li, "Enhancement of the modulation bandwidth for GaN-based lightemitting diode by surface plasmons," Opt. Express, vol. 23, no. 11, pp. 13752-13760, 2015.

[122] C. Yang, A. A. Bettiol, Y. Shi, M. Bosman, H. R. Tan, W. P. Goh, J. H. Teng, and E. J. Teo, "Fast electrical modulation in a plasmonic-enhanced, V-Pit-Textured, light-emitting diode," Adv. Opt. Mater., vol. 3, no. 12, pp. 1703-1709, 2015.

[123] F. R. Gfeller and U. Bapst, "Wireless in-house data communication via diffuse infrared radiation," Proc. IEEE, vol. 67, no. 11, pp. 1474-1486, 1979.

[124] M. Kavehrad, "Optical wireless applications: A solution to ease the wireless airwaves spectrum crunch," in Proc. SPIE 8645, Broadband Access Communication Technologies VII, 86450G, 2013, vol. 8645, p. 86450G-1.

[125] X. Li, N. Bamiedakis, X. Guo, J. McKendry, E. Xie, R. Ferreira, E. Gu, M. Dawson, R. Penty, and I. White, "Wireless visible light communications employing feed-forward pre-equalization and PAM-4 modulation," J. Light. Technol., vol. 34, no. 8, pp. 2049-2055, 2016.

[126] C. Shen, T. K. Ng, J. T. Leonard, A. Pourhashemi, H. M. Oubei, M. S. Alias, S. Nakamura, S. P. DenBaars, J. S. Speck, A. Y. Alyamani, M. M. Eldesouki, and B. S. Ooi, "High-modulation-efficiency, integrated waveguide modulator-laser diode at 448 nm," ACS Photonics, vol. 3, no. 2, pp. 262-268, 2016.

[127] B. Janjua, H. M. Oubei, J. R. D. Retamal, T. K. Ng, C.-T. Tsai, H.-Y. Wang, Y.-C. Chi, H.-C. Kuo, G.-R. Lin, J.-H. He, and B. S. Ooi, "Going beyond 4 Gbps data rate by employing RGB laser diodes for visible light communication," Opt. Express, vol. 23, no. 14, pp. 18746-18753, 2015.

[128] Y.-C. Chi, D.-H. Hsieh, C.-T. Tsai, H.-Y. Chen, H.-C. Kuo, and G.-R. Lin, “450-nm GaN laser diode enables high-speed visible light communication with 9-Gbps QAM-OFDM,” Opt. Express, vol. 23, no. 10, pp. 13051-13059, 2015.

[129] G. Cossu, A. Wajahat, R. Corsini, and E. Ciaramella, "5.6 Gbit/s downlink and 1.5 Gbit/s uplink optical wireless transmission at indoor distances ( $\geq 1.5 \mathrm{~m}$ ), " in Optical Communication (ECOC), 2014 European Conference on, 2014, pp. 1-3.

[130] Y. Wang, L. Tao, X. Huang, J. Shi, and N. Chi, "Enhanced performance of a high-speed WDM CAP64 VLC system employing 
Volterra series-based nonlinear equalizer," IEEE Photonics J., vol. 7, no. 3, pp. 1-7, 2015.

[131] J. R. D. Retamal, H. M. Oubei, B. Janjua, Y.-C. Chi, H.-Y. Wang, C.-T. Tsai, T. K. Ng, D.-H. Hsieh, H.-C. Kuo, M.-S. Alouini, J.-H. He, G.-R. Lin, and B. S. Ooi, "4-Gbit/s visible light communication link based on 16-QAM OFDM transmission over remote phosphor-film converted white light by using blue laser diode," Opt. Express, vol. 23, no. 26, pp. 33656-33666, 2015.

[132] C. Lee, C. Shen, H. M. Oubei, M. Cantore, B. Janjua, T. K. Ng, R. M. Farrell, M. M. El-Desouki, J. S. Speck, S. Nakamura, B. S. Ooi, and S. P. DenBaars, "2 Gbit/s data transmission from an unfiltered laser-based phosphor-converted white lighting communication system," Opt. Express, vol. 23, no. 23, pp. 29779-29787, 2015.

[133] X. Huang, S. Chen, Z. Wang, J. Shi, Y. Wang, J. Xiao, and N. Chi, "2.0-Gb/s visible light link based on adaptive bit allocation OFDM of a single phosphorescent white LED,” IEEE Photonics J., vol. 7, no. 5, pp. 1-8, 2015.

[134] X. Huang, Z. Wang, J. Shi, Y. Wang, and N. Chi, "1.6 Gbit/s phosphorescent white LED based VLC transmission using a cascaded pre-equalization circuit and a differential outputs PIN receiver," Opt. Express, vol. 23, no. 17, pp. 22034-22042, 2015.

[135] Nan Chi, Yiguang Wang, and Xingxing Huang, "Advancing the capacity of phosphorescent white LED based visible light communication network," 2015 IEEE Summer Top. Meet. Ser., vol. 2, pp. 33-34, 2015.

[136] X. Huang, J. Shi, J. Li, Y. Wang, and N. Chi, “A Gb/s VLC transmission using hardware preequalization circuit,” IEEE Photonics Technol. Lett., vol. 27, no. 18, pp. 1915-1918, 2015.

[137] G. Stepniak, L. Maksymiuk, and J. Siuzdak, "1.1 Gbit/s white lighting LED-based visible light link with pulse amplitude modulation and Volterra DFE equalization,” Microw. Opt. Technol. Lett., vol. 57, no. 7, pp. 1620-1622, Jul. 2015.

[138] H. Xingxing, S. Jianyang, L. Jiehui, W. Yiguang, W. Yuanquan, and C. Nan, "750Mbit/s visible light communications employing 64QAM-OFDM based on amplitude equalization circuit," in Optical Fiber Communications Conference and Exhibition (OFC), 2015, 2015, pp. 1-3.

[139] H. Li, Y. Zhang, X. Chen, C. Wu, J. Guo, Z. Gao, W. Pei, and H. Chen, "682 Mbit/s phosphorescent white LED visible light communications utilizing analog equalized 16QAM-OFDM modulation without blue filter,” Opt. Commun., vol. 354, pp. 107$111,2015$.

[140] J. Li, Y. Xu, J. Shi, Y. Wang, X. Ji, H. Ou, and N. Chi, "A $2 \times 2$ imaging MIMO system based on LED Visible Light Communications employing space balanced coding and integrated PIN array reception," Opt. Commun., vol. 367, pp. 214-218, 2016.

[141] T. Chen, L. Liu, Z. Zheng, J. Song, K. Wu, and W. Hu, "Fisheye-lens-based space division multiplexing system for visible light communications," EURASIP J. Wirel. Commun. Netw., vol. 2015, no. 1, pp. 1-7, 2015.

[142] Y. Wang, C. Yang, Y. Wang, and N. Chi, "Gigabit polarization division multiplexing in visible light communication," Opt. Lett., vol. 39, no. 7, pp. 1823-1826, 2014.

[143] W. Yuanquan, C. Nan, Y. Wang, and N. Chi, "Demonstration of high-speed 2x2 non-imaging MIMO Nyquist single carrier visible light communication with frequency domain equalization,” Light. Technol. J., vol. 32, no. 11, pp. $2087-2093,2014$.

[144] A. Burton, H. Minh, Z. Ghassemlooy, E. Bentley, and C. Botella, "Experimental demonstration of 50-Mbps visible light communications using $4 \times 4$ MIMO,” IEEE Photonics Technol. Lett., vol. 26, 2014.

[145] Y. Wang, X. Huang, J. Zhang, Y. Wang, and N. Chi, "Enhanced performance of visible light communication employing 512QAM N-SC-FDE and DD-LMS,” Opt. Express, vol. 22, no. 13, pp. 15328-15334, 2014.

[146] L. Honglei, C. Xiongbin, H. Beiju, T. Danying, C. Hongda, H. Li, X. Chen, B. Huang, D. Tang, and H. Chen, "bandwidth visible light communications based on a post-equalization circuit," IEEE Photonics Technol. Lett., vol. 26, no. 2, pp. 119-122, 2014.

[147] H. Li, X. Chen, J. Guo, and H. Chen, “A $550 \mathrm{Mbit} / \mathrm{s}$ real-time visible light communication system based on phosphorescent white light LED for practical high-speed low-complexity application,” Opt. Express, vol. 22, no. 22, pp. 27203-27213, 2014.

[148] S. Watson, M. Tan, S. P. Najda, P. Perlin, M. Leszczynski, G. Targowski, S. Grzanka, and A. E. Kelly, "Visible light communications using a directly modulated 422 nm GaN laser diode," Opt. Lett., vol. 38, no. 19, pp. 3792-3794, 2013.

[149] P. H. Binh, V. D. Trong, D. D. Hung, P. Renucci, A. Balocchi, and X. Marie, "Demonstration of $300 \mathrm{Mbit} / \mathrm{s}$ free space optical link with commercial visible LED," in New Circuits and Systems Conference (NEWCAS), 2013 IEEE 11th International, 2013, pp. 13 .

[150] L. Grobe, A. Paraskevopoulos, J. Hilt, D. Schulz, F. Lassak, F. Hartlieb, C. Kottke, V. Jungnickel, and K. D. Langer, "High-speed visible light communication systems," IEEE Commun. Mag., vol. 51, no. 12, pp. 60-66, 2013.

[151] N. Fujimoto and H. Mochizuki, "477 Mbit/s visible light transmission based on OOK-NRZ modulation using a single commercially available visible LED and a practical LED driver with a pre-emphasis circuit," in Optical Fiber Communication Conference and Exposition and the National Fiber Optic Engineers Conference (OFC/NFOEC), 2013, 2013, pp. 1-3.

[152] S. Rajbhandari, P. A. Haigh, Z. Ghassemlooy, and W. Popoola, "Wavelet-neural network VLC receiver in the presence of artificial light interference," Photonics Technol. Lett. IEEE, vol. 25, no. 15, pp. 1424-1427, 2013.

[153] P. A. Haigh, Z. Ghassemlooy, S. Rajbhandari, I. Papakonstantinou, and W. Popoola, "Visible light communications: $170 \mathrm{Mb} / \mathrm{s}$ using an artificial neural network equalizer in a low bandwidth white light configuration," Light. Technol. J., vol. 32, no. 9, pp. $1807-1813,2014$.

[154] F.-M. Wu, C.-T. Lin, C.-C. Wei, C.-W. Chen, Z.-Y. Chen, and K. Huang, "3.22-Gb/s WDM visible light communication of a single RGB LED employing carrier-less amplitude and phase modulation," in Optical Fiber Communication Conference/National Fiber Optic Engineers Conference 2013, 2013, p. OTh1G.4.

[155] F. M. Wu, C. T. Lin, C. C. Wei, C. W. Chen, Z. Y. Chen, H. T. Huang, and S. Chi, "Performance comparison of OFDM signal and CAP signal over high capacity RGB-LED-based WDM visible light communication," IEEE Photonics J., vol. 5, no. 4, 2013.

[156] Z. Shuailong, S. Watson, J. J. D. McKendry, D. Massoubre, A. Cogman, G. Erdan, R. K. Henderson, A. E. Kelly, and M. D. Dawson, "1.5 Gbit/s multi-channel visible light communications using CMOS-controlled GaN-based LEDs," J. Light. Technol., vol. 31, no. 8, pp. 1211-1216, 2013.

[157] A. H. Azhar, T. Tran, and D. C. O’Brien, "A gigabit/s indoor wireless transmission using MIMO-OFDM visible-light communications," IEEE Photonics Technol. Lett., vol. 25, no. 2, pp. 171-174, 2013.

[158] Y. Wang, Y. Wang, N. Chi, J. Yu, and H. Shang, "Demonstration of 575-Mb/s downlink and 225-Mb/s uplink bi-directional SCM-WDM visible light communication using RGB LED and phosphor-based LED," Opt. Express, vol. 21, no. 1, pp. 12031208, 2013.

[159] G. Cossu, A. M. Khalid, P. Choudhury, R. Corsini, and E. Ciaramella, "3.4 Gbit/s visible optical wireless transmission based on RGB LED," Opt. Express, vol. 20, 2012.

[160] G. Cossu, A. M. Khalid, P. Choudhury, R. Corsini, and E. Ciaramella, "2.1 Gbit/s visible optical wireless transmission," in Optical Communications (ECOC), 2012 38th European Conference and Exhibition on, 2012, pp. 1-3.

[161] F. Wu, C. Lin, C. Wei, and C. Chen, "1.1-Gb/s white-LED-based visible light communication employing carrier-less amplitude and phase modulation,” IEEE Photonics Technol. Lett., vol. 24, no. 19, pp. 1730-1732, 2012.

[162] A. M. Khalid, G. Cossu, R. Corsini, P. Choudhury, and E. Ciaramella, "1-Gb/s transmission over a phosphorescent white LED by 
using rate-adaptive discrete multitone modulation," IEEE Photonics J., vol. 4, no. 5, pp. 1465-1473, 2012.

[163] N. Fujimoto and H. Mochizuki, "614 Mbit/s OOK-based transmission by the duobinary technique using a single commercially available visible LED for high-speed visible light communications,” Opt. Commun. (ECOC), 2012 38th Eur. Conf. Exhib., pp. 1$3,2012$.

[164] C. Kottke, J. Hilt, K. Habel, J. Vucic, and K. D. Langer, "1.25 Gbit/s visible light WDM link based on DMT modulation of a single RGB LED luminary,” in Optical Communications (ECOC), 2012 38th European Conference and Exhibition on, 2012, pp. $1-3$.

[165] J. Vucic, C. Kottke, K. Habel, and K.-D. Langer, "803 Mbit/s visible light WDM link based on DMT modulation of a single RGB LED luminary," in 2011 Optical Fiber Communication Conference and Exposition and the National Fiber Optic Engineers Conference, 2011, pp. 1-3.

[166] J. Vucic, C. Kottke, S. Nerreter, K. Langer, and J. W. Walewski, "513 Mbit/s visible light communications link based on DMTmodulation of a white LED," Light. Technol. J., vol. 28, no. 24, pp. 3512-3518, 2010.

[167] J. Vucic, C. Kottke, S. Nerreter, K. Habel, A. Buttner, K.-D. Langer, and J. W. Walewski, "230 Mbit/s via a wireless visible-light link based on OOK modulation of phosphorescent white LEDs," Opt. Fiber Commun. (OFC), collocated Natl. Fiber Opt. Eng. Conf. 2010 Conf., pp. 7-9, 2010.

[168] J. Vǔcić, C. Kottke, S. Nerreter, A. Büttner, K. D. Langer, and J. W. Walewski, "White light wireless transmission at 200+ Mb/s net data rate by use of discrete-multitone modulation," IEEE Photonics Technol. Lett., vol. 21, no. 20, pp. 1511-1513, 2009.

[169] J. Vucic, C. Kottke, S. Nerreter, K. Habel, a. Buttner, K.-D. D. Langer, and J. W. Walewski, "125 Mbit/s over 5 m wireless distance by use of OOK-Modulated phosphorescent white LEDs," in Optical Communication, 2009. ECOC '09. 35th European Conference on, 2009, no. 1, pp. 1-2.

[170] H. Le Minh, D. O’Brien, G. Faulkner, L. Zeng, K. Lee, D. Jung, and Y. J. Oh, "High-speed visible light communications using multiple-resonant equalization," IEEE Photonics Technol. Lett., vol. 20, no. 14, pp. 1243-1245, 2008.

[171] J. Grubor, S. C. J. Lee, K.-D. Langer, T. Koonen, and J. W. Walewski, "Wireless High-Speed Data Transmission with Phosphorescent White-Light LEDs," in Optical Communication - Post-Deadline Papers (published 2008), 2007 33rd European Conference and Exhibition of, 2007, pp. 1-2.

[172] M. Z. Afgani, H. Haas, H. Elgala, and D. Knipp, "Visible light communication using OFDM," in 2nd International Conference on Testbeds and Research Infrastructures for the Development of Networks and Communities, 2006. TRIDENTCOM 2006., 2006, pp. $129-134$.

[173] T. Komine and M. Nakagawa, "Integrated system of white LED visible-light communication and power-line communication," in IEEE International Symposium on Personal, Indoor and Mobile Radio Communications, PIMRC, 2002, vol. 4, pp. 1762-1766.

[174] J. Armstrong, "OFDM for optical communications," Light. Technol. J., vol. 27, no. 3, pp. 189-204, 2009.

[175] T. Lueftner, C. Kroepl, M. Huemer, J. Hausner, R. Hagelauer, and R. Weigel, "Edge-position modulation for high-speed wireless infrared communications," IEE Proc. Optoelectron., vol. 150, no. 5, pp. 427-437, 2003.

[176] J. Armstrong, B. Schmidt, D. Kalra, H. A. Suraweera, and A. J. Lowery, "Performance of Asymmetrically clipped optical OFDM in AWGN for an Intensity modulated direct detection system," in Global Telecommunications Conference, 2006. GLOBECOM '06. IEEE, 2006, pp. 1-5.

[177] E. Monteiro and S. Hranilovic, "Design and implementation of color-shift keying for visible light communications," Light. Technol. J., vol. 32, no. 10, pp. 2053-2060, 2014.

[178] S. Randel, F. Breyer, S. C. J. Lee, and J. W. Walewski, “Advanced modulation schemes for short-range optical communications," IEEE J. Sel. Top. Quantum Electron., vol. 16, no. 5, pp. 1280-1289, 2010.

[179] J. R. Barry, Wireless infrared communications. Boston: Kluwer Academic Publishers, 1994.

[180] S. Loquai, R. Kruglov, B. Schmauss, C. Bunge, F. Winkler, O. Ziemann, E. Hartl, and T. Kupfer, "Comparison of modulation schemes for $10.7 \mathrm{~Gb} / \mathrm{s}$ transmission over large-core $1 \mathrm{~mm}$ PMMA polymer optical fiber," J. Light. Technol., vol. 31, no. 13, pp. 2170-2176, 2013.

[181] S. H. Yang, D. H. Kwon, and S. K. Han, "Overcoming bandwidth limitation of LED by using multilevel differential PAM in VLC," in Progress In Electrmagnetics Research Symposium, 2015, pp. 1397-1401.

[182] J. F. Li, Z. T. Huang, R. Q. Zhang, F. X. Zeng, M. Jiang, and Y. F. Ji, "Superposed pulse amplitude modulation for visible light communication," Opt. Express, vol. 21, no. 25, pp. 31006-31011, 2013.

[183] R. A. Cryan and R. T. Unwin, "Optical space communications employing pulse position modulation," in IEE Colloquium on Advanced Modulation and Coding Techniques for Satellite Communications, 1992, pp. 7/1-7/5.

[184] Y. Kawamura and T. Tanaka, "Transmission of the LED light from the space to the ground," AIP Adv., vol. 3, no. 10, pp. 0-6, 2013.

[185] J. Grubor, S. Lee, and K. Langer, "Wireless high-speed data transmission with phosphorescent white light LEDs," in Ecoc 2007, 2007, vol. 1, no. 1, pp. 3-4.

[186] H. Park and J. R. Barry, "Performance of multiple pulse position modulation on multipath channels," Optoelectron. IEE Proc. -, vol. 143 , no. 6 , pp. 360-364, 1996.

[187] H. Sugiyama and K. Nosu, "MPPM: a method for improving the band-utilization efficiency in optical PPM," Light. Technol. J., vol. 7, no. 3, pp. 465-472, 1989.

[188] D. Shiu and J. M. Kahn, "Differential pulse position modulation for power-efficient optical communication," IEEE Trans. Commun., vol. 47, no. 8, pp. 1201-1210, 1999.

[189] M. Noshad and M. Brandt-Pearce, "Application of expurgated PPM to indoor visible light communications -Part I: Single-user systems," J. Light. Technol., vol. 32, no. 5, pp. 883-890, 2014.

[190] W. C. Palsy, "A VDSL Tutorial," Luleå Tekniska Univ., Luleå, Sweden, Tech. Rep., 2000, 2010. [Online]. Available: http://epubl.1tu.se/1402-1528/2000/02/LTU-FR-0002-SE.pdf. [Accessed: 26-May-2016].

[191] M. I. Olmedo, T. Zuo, J. B. Jensen, Q. Zhong, X. Xu, S. Popov, and I. T. Monroy, "Multiband carrierless amplitude phase modulation for high capacity optical data links," J. Light. Technol., vol. 32, no. 4, pp. 798-804, 2014.

[192] J. Gao, Y. H. L. Y. H. Leung, and V. Sreeram, "Digital filters for carrierless amplitude and phase receivers," in Proceedings of IEEE Region 10 International Conference on Electrical and Electronic Technology. TENCON 2001 (Cat. No.01CH37239), 2001, vol. 2 , no. 446 , pp. $575-579$.

[193] A. H. Abdolhamid and D. A. Johns, "A comparison of CAP/QAM architectures," in ISCAS '98. Proceedings of the 1998 IEEE International Symposium on Circuits and Systems (Cat. No.98CH36187), vol. 4, p. 316,-316/3.

[194] L. Tao, Y. Wang, Y. Gao, A. P. T. Lau, N. Chi, and C. Lu, "Experimental demonstration of $10 \mathrm{~Gb} / \mathrm{s}$ multi-level carrier-less amplitude and phase modulation for short range optical communication systems.," Opt. Express, vol. 21, no. 5, pp. 6459-65, Mar. 2013.

[195] J. Gao and Y. H. Leung, “A new adaptive equalizer for carrierless amplitude and phase (CAP) receivers,” ISCAS'99. Proc. 1999 
IEEE Int. Symp. Circuits Syst. VLSI (Cat. No.99CH36349), vol. 3, pp. 90-93, 1999.

[196] P. Haigh, A. Burton, K. Werfli, H. Minh, E. Bentley, P. Chvojka, W. Popoola, I. Papakonstantinou, and S. Zvanovec, "A multiCAP visible light communications system with 4.85 b/s/Hz spectral efficiency," IEEE J. Sel. Areas Commun., vol. 33, no. 9, p. 1, 2015.

[197] Y. Wang, L. Tao, Y. Wang, and N. Chi, "High speed WDM VLC system based on multi-band CAP64 with weighted preequalization and modified CMMA based post-equalization," IEEE Commun. Lett., vol. 18, no. 10, pp. 1719-1722, 2014.

[198] S. Dimitrov, S. Sinanovic, and H. Haas, "Signal shaping and modulation for optical wireless communication," J. Light. Technol., vol. 30, no. 9, pp. 1319-1328, 2012.

[199] D. Tsonev, S. Videv, and H. Haas, "Unlocking spectral efficiency in intensity modulation and direct detection systems," IEEE J. Sel. Areas Commun., vol. 33, no. 9, pp. 1758-1770, 2015

[200] H. Elgala, R. Mesleh, and H. Haas, "Non-linearity effects and predistortion in optical OFDM wireless transmission using LEDs," Int. J. Ultra Wideband Commun. Syst., vol. 1, no. 2, pp. 143-150, 2009.

[201] J. Armstrong and B. J. C. Schmidt, "Comparison of asymmetrically clipped optical OFDM and DC-biased optical OFDM in AWGN," Commun. Lett. IEEE, vol. 12, no. 5, pp. 343-345, 2008.

[202] D. Tsonev and H. Haas, "Avoiding spectral efficiency loss in unipolar OFDM for optical wireless communication," 2014 IEEE Int. Conf. Commun. ICC 2014, pp. 3336-3341, 2014.

[203] N. Fernando, Y. Hong, and E. Viterbo, "Flip-OFDM for unipolar communication systems," IEEE Trans. Commun., vol. 60, no. 12, pp. 3726-3733, Dec. 2012.

[204] D. Tsonev, S. Sinanovic, and H. Haas, "Complete modeling of nonlinear distortion in OFDM-based optical wireless communication,” J. Light. Technol., vol. 31, no. 18, pp. 3064-3076, 2013.

[205] A. H. Azhar and D. O'Brien, "Experimental comparisons of optical OFDM approaches in visible light communications," 2013 IEEE Globecom Work. GC Wkshps 2013, pp. 1076-1080, 2013.

[206] Z. Yu, R. J. Baxley, and G. T. Zhou, "Achievable data rate analysis of clipped FLIP-OFDM in optical wireless communication," 2012 IEEE Globecom Work. GC Wkshps 2012, no. 3, pp. 1203-1207, 2012.

[207] D. O'Brien, H. Haas, S. Rajbhandari, H. Chun, G. Faulkner, K. Cameron, A. V. N. Jalajakumari, R. Henderson, D. Tsonev, M. Ijaz, Z. Chen, E. Xie, J. J. D. McKendry, J. Herrnsdorf, E. Gu, and M. D. Dawson, "Integrated multiple-input multiple-output visible light communications systems: recent progress and results," in Proc. SPIE 9387, Broadband Access Communication Technologies IX, 2015, vol. 9387, p. 93870P-93870P-6.

[208] Y. Wang, Y. Wang, and N. Chi, "Experimental verification of performance improvement for a gigabit wavelength division multiplexing visible light communication system utilizing asymmetrically clipped optical orthogonal frequency division multiplexing," Photonics Res., vol. 2, no. 5, p. 138, Sep. 2014.

[209] J. L. Wei, J. D. Ingham, D. G. Cunningham, R. V. Penty, and I. H. White, "Performance and power dissipation comparisons between $28 \mathrm{~Gb} / \mathrm{s}$ NRZ, PAM, CAP and optical OFDM systems for data communication applications," J. Light. Technol., vol. 30, no. 20, pp. 3273-3280, 2012.

[210] E. Vanin, "Performance evaluation of intensity modulated optical OFDM system with digital baseband distortion.," Opt. Express, vol. 19, no. 5, pp. 4280-4293, 2011.

[211] G. Stepniak, M. Schuppert, and C. A. Bunge, "Advanced modulation formats in phosphorous LED VLC links and the impact of blue filtering," Light. Technol. J., vol. 33, no. 21, pp. 4413-4423, 2015.

[212] G. Stepniak, L. Maksymiuk, and J. Siuzdak, "Experimental comparison of PAM, CAP, and DMT modulations in phosphorescent white LED transmission link," Photonics Journal, IEEE, vol. 7, no. 3, pp. 1-8, 2015.

[213] H. Le Minh, S.-K. Liaw, N. Aslam, E. Bentley, Z. Ghassemlooy, and A. Burton, "Experimental demonstration of a 10BASE-T Ethernet visible light communications system using white phosphor light-emitting diodes," IET Circuits, Devices Syst., vol. 8, no. 4, pp. 322-330, 2014.

[214] H. Li, X. Chen, J. Guo, D. Tang, B. Huang, and H. Chen, "200 Mb/s visible optical wireless transmission based on NRZ-OOK modulation of phosphorescent white LED and a pre-emphasis circuit," Chinese Opt. Lett., vol. 12, no. 10, p. 100604, 2014.

[215] Honglei Li; Xiongbin Chen; Junqing Guo; Zongyu Gao; Hongda Chen, H. Li, X. Chen, J. Guo, Z. Gao, and H. Chen, “An analog modulator for $460 \mathrm{Mb} / \mathrm{s}$ visible light data transmission based on OOK-NRZ modulation,” IEEE Wirel. Commun., vol. 22, no. 2, pp. 68-73, Apr. 2015.

[216] N. Fujimoto and S. Yamamoto, "The fastest visible light transmissions of $662 \mathrm{Mb} / \mathrm{s}$ by a blue LED, $600 \mathrm{Mb} / \mathrm{s}$ by a red LED, and $520 \mathrm{Mb} / \mathrm{s}$ by a green LED based on simple OOK-NRZ modulation of a commercially available RGB-type white LED using preemphasis and post-equalizing techniques," Eur. Conf. Opt. Commun. ECOC, no. Cml, pp. 3-5, 2014.

[217] T. Kishi, H. Tanaka, Y. Umeda, and O. Takyu, "A high-speed LED driver that sweeps out the remaining carriers for visible light communications," J. Light. Technol., vol. 32, no. 2, pp. 239-249, 2014.

[218] C. H. Yeh, C. W. Chow, H. Y. Chen, J. Chen, and Y. L. Liu, “Adaptive 84.44-190 Mbit/s phosphor-LED wireless communication utilizing no blue filter at practical transmission distance," Opt Express, vol. 22, no. 8, pp. 9783-9788, 2014.

[219] H. Chou, S. Liaw, C. Teng, J. Jiang, C. Tsai, C. Wu, and M. Chien, "RGB LEDs visible light communications based on equalized receiver with broadband optical filters," in Fiber Optics in Access Network (FOAN), 2015 International Workshop on, 2015, pp. $22-25$.

[220] J. Shi, X. Huang, Y. Wang, and N. Chi, "Real-time bi-directional visible light communication system utilizing a phosphor-based LED and RGB LED," in 2014 6th International Conference on Wireless Communications and Signal Processing, WCSP 2014, 2014.

[221] K. Azadet, E. F. Haratsch, H. Kim, F. Saibi, J. H. Saunders, M. Shaffer, and L. Song, "Equalization and FEC techniques for optical transceivers," IEEE J. Solid-State Circuits, vol. 37, no. 3, pp. 317-327, Mar. 2002.

[222] J. G. Proakis, Digital Communications. New York: McGraw-Hill, 2004.

[223] J. M. Kahn, W. J. Krause, and J. B. Carruthers, "Experimental characterization of non-directed indoor infrared channels," IEEE Trans. Commun., vol. 43, no. 234, pp. 1613-1623, 1995.

[224] C. H. Yeh, Y. F. Liu, C. W. Chow, Y. Liu, P. Y. Huang, and H. K. Tsang, "Investigation of 4-ASK modulation with digital filtering to increase 20 times of direct modulation speed of white-light LED visible light communication system," Opt. Express, vol. 20 , no. 15 , p. 16218,2012

[225] P. A. Haigh, Z. Ghassemlooy, S. Rajbhandari, and E. Leitgeb, “A $100 \mathrm{Mb} / \mathrm{s}$ visible light communications system using a linear adaptive equalizer," in 2014 19th European Conference on Networks and Optical Communications - (NOC), 2014, pp. 136-139.

[226] A. Hussain, J. J. Soraghan, and T. S. Durrani, "A new adaptive functional-link neural-network-based DFE for overcoming cochannel interference," IEEE Trans. Commun., vol. 45, no. 11, pp. 1358-1362, 1997.

[227] M. Zhanga, Y. Zhanga, and N. Haoa, "Visible light communications using blind equalization," in Communications and Photonics Conference and Exhibition, 2011. ACP. Asia, 2011, vol. 8309, pp. 1-6. 
[228] S. Haykin, "Adaptive digital communication receivers," IEEE Commun. Mag., vol. 38, no. 12, pp. 106-114, 2002.

[229] S. Haykin, J. Nie, and B. Currie, "Neural network-based receiver for wireless communications," IEE Electron. Lett., vol. 35, no. 3, pp. 203-204, 1999.

[230] G. Stepniak, J. Siuzdak, and P. Zwierko, "Compensation of a VLC phosphorescent white LED nonlinearity by means of Volterra DFE,” IEEE Photonics Technol. Lett., vol. 25, no. 16, pp. 1597-1600, 2013

[231] G.-K. C. and G. T. Z. Kai Ying, Zhenhua Yu, R. J. Baxley, Hua Qian, "Nonlinear distortion mitigation in visible light communications," IEEE Wirel. Commun., vol. 22, no. 2, pp. 36-45, 2015.

[232] H. Qian, S. J. Yao, S. Z. Cai, and T. Zhou, "Adaptive postdistortion for nonlinear LEDs in visible light communications," IEEE Photonics J., vol. 6, no. 4, 2014.

[233] R. Mitra and V. Bhatia, "Chebyshev polynomial based adaptive predistorter for nonlinear LED compensation in VLC," IEEE Photonics Technol. Lett., vol. 1135, no. 2, pp. 1-1, May 2016.

[234] Y. Wang, J. Shi, C. Yang, Y. Wang, and N. Chi, "Integrated $10 \mathrm{~Gb} / \mathrm{s}$ multilevel multiband passive optical network and 500 Mb/s indoor visible light communication system based on Nyquist single carrier frequency domain equalization modulation.," Opt. Lett., vol. 39, no. 9, pp. 2576-9, May 2014.

[235] S. Long, M. Khalighi, M. Wolf, Z. Ghassemlooy, and S. Bourennane, "Performance of carrier-less amplitude and phase modulation with frequency domain equalization for indoor visible light communications," in 4th International Workshop on Optical Wireless Communications (IWOW), 2015, 2015, pp. 16-20.

[236] Y. Wang, R. Li, Y. Wang, and Z. Zhang, "3.25-Gbps visible light communication system based on single carrier frequency domain equalization utilizing an RGB LED," in Optical Fiber Communication Conference, OSA Technical Digest, 2014 , no. Th1F.1., pp. 3-5.

[237] A. Nuwanpriya, S.-W. Ho, J. Zhang, A. Grant, and L. Luo, "PAM-SCFDE for optical wireless communications," J. Light. Technol., vol. 33, no. 14, pp. 2938 - 2949, 2015.

[238] M. Wolf and M. Haardt, "Comparison of OFDM and frequency domain equalization for dispersive optical channels with direct detection," in International Conference on Transparent Optical Networks, 2012, pp. 1-7.

[239] M. A. Kashani and M. Kavehrad, "On the performance of single- and multi-carrier modulation schemes for indoor visible light communication systems," in Proceedings of Globecom, Austin, TX: IEEE, 2014, pp. 2084-2089.

[240] J. Li, Z. Huang, X. Liu, and Y. Ji, "Hybrid time-frequency domain equalization for LED nonlinearity mitigation in OFDM-based VLC systems," Opt. Express, vol. 23, no. 1, pp. 611-9, 2015.

[241] T. Fath and H. Haas, "Performance comparison of MIMO techniques for optical wireless communications in indoor environments," IEEE Trans. Commun., vol. 61, 2013.

[242] D. O’Brien, R. Turnbull, H. Le Minh, G. Faulkner, O. Bouchet, P. Porcon, M. El Tabach, E. Gueutier, M. Wolf, L. Grobe, and J. Li, "High-speed optical wireless demonstrators: Conclusions and future directions," J. Light. Technol., vol. 30, no. 13, pp. 21812187, 2012.

[243] Z. Chen, D. Tsonev, and H. Haas, "Improving SINR in indoor cellular visible light communication networks," in 2014 IEEE International Conference on Communications, ICC 2014, 2014, no. 1, pp. 3383-3388.

[244] D. C. O’Brien, G. E. Faulkner, E. B. Zyambo, K. Jim, D. J. Edwards, P. Stavrinou, G. Parry, J. Bellon, M. J. Sibley, V. A Lalithambika, V. M. Joyner, R. J. Samsudin, D. M. Holburn, and R. J. Mears, "Integrated transceivers for optical wireless communications," IEEE J. Sel. Top. Quantum Electron., vol. 11, no. 1, pp. 173-183, 2005.

[245] A. Tsiatmas, F. M. J. Willems, and S. Baggen, "Optimum Diversity Combining Techniques for Visible Light Communication Systems," in Globecom 2014 Workshop - Optical Wireless Communications, 2014, vol. 1, pp. 541-546.

[246] J. Li, X. Huang, and N. Chi, "Enhanced performance of single-input multiple-output visible light communication system utilizing space diversity technology," Opt. Eng., vol. 54, no. 3, p. 036109, Mar. 2015.

[247] J. Li, X. Huang, and N. Chi, "High speed wireless access based on visible light communication utilizing maximum ratio combination of multi-detectors," in International Conference on Ambient Computing, Applications, Services and Technologies High, 2014, pp. 39-42.

[248] A. Burton, Z. Ghassemlooy, S. Rajbhandari, and S.-K. Liaw, "Design and analysis of an angular-segmented full-mobility visible light communications receiver," Trans. Emerg. Telecommun. Technol., vol. 25, no. 6, pp. 591-599, Jun. 2014.

[249] K. D. Dambul, D. O’Brien, and G. Faulkner, "Indoor optical wireless MIMO System with an imaging receiver," IEEE Photonics Technol. Lett., vol. 23, no. 2, pp. 97-99, 2011

[250] J. Herrnsdorf, J. J. D. Mckendry, R. Ferreira, R. Henderson, S. Videv, S. Watson, H. Haas, A. E. Kelly, E. Gu, and M. D. Dawson, "Single-chip discrete multitone generation," in Summer Topicals Meeting Series (SUM), 2015, 2015, vol. 2, pp. 47-48.

[251] X. Li, N. Bamiedakis, J. L. Wei, J. J. D. Mckendry, E. Xie, R. Ferreira, and E. Gu, "6.25 Gb/s POF link using GaN $\mu$ LED arrays and optically generated pulse amplitude modulation," in 2015 Conference on Lasers and Electro-Optics (CLEO), 2015, pp. 1-2.

[252] A. Yang, Y. Wu, M. Kavehrad, and G. Ni, "Grouped modulation scheme for LED array module in a visible light communication system,” IEEE Wirel. Commun., vol. 22, no. 2, pp. 24-28, 2015.

[253] M. S. A. Mossaad and S. Hranilovic, "Practical OFDM signalling for visible light communications using spatial summation," 2014 27th Bienn. Symp. Commun. QBSC 2014, pp. 5-9, 2014.

[254] B. Yu, H. Zhang, L. Wei, and J. Song, "Subcarrier grouping OFDM for visible-light communication systems," IEEE Photonics J., vol. 7, no. 5, pp. 1-12, 2015.

[255] R. Mesleh, H. Elgala, and T. Little, "A novel method to mitigate LED nonlinearity distortions in optical wireless OFDM systems," in Optical Fiber Communication Conference/National Fiber Optic Engineers Conference 2013, 2013, p. JW2A.69.

[256] D. F. Zhang, Y. J. Zhu, and Y. Y. Zhang, "Multi-LED phase-shifted OOK modulation based visible light communication systems," IEEE Photonics Technol. Lett., vol. 25, no. 23, pp. 2251-2254, 2013.

[257] A. Dobesch, L. N. Alves, and O. Wilfert, "Spatial ODAC performance for indoor environment," in 2014 3rd International Workshop in Optical Wireless Communications (IWOW), 2014, pp. 70-74

[258] D. Tse and P. Viswanath, Fundamentals of Wireless Communications. Cambridge, UK: University Press, 2006.

[259] M. Di Renzo, H. Haas, A. Ghrayeb, S. Sugiura, L. Hanzo, M. Di Renzo, H. Haas, A. Ghrayeb, S. Sugiura, and L. Hanzo, "Spatial modulation for generalized MIMO: Challenges, opportunities, and implementation," Proc. IEEE, vol. 102, no. 1, pp. 56-103, 2014.

[260] W. O. Popoola and H. Haas, "Demonstration of the merit and limitation of generalised space shift keying for indoor visible light communications," J. Light. Technol., vol. 32, no. 10, pp. 1960-1965, 2014

[261] W. O. Popoola, E. Poves, and H. Haas, "Error performance of generalised space shift keying for indoor visible light communications," IEEE Trans. Commun., vol. 61, no. 5, pp. 1968-1976, 2013.

[262] M. Ijaz, D. Tsonev, J. J. D. McKendry, E. Xie, S. Rajbhandari, H. Chun, G. Faulkner, E. Gu, M. D. Dawson, D. O’Brien, and H. Haas, "Experimental proof-of-concept of optical spatial modulation OFDM using micro LEDs," in 2015 IEEE International 
Conference on Communication Workshop, ICCW 2015, 2015, vol. 2, pp. 1338-1343.

[263] K. Cai and M. Jiang, "SM/SPPM aided multiuser precoded visible light communication systems," IEEE Photonics J., vol. 8, no. 2, pp. 1-9, 2016.

[264] T. Fath and H. Haas, "Optical spatial modulation using colour LEDs,” 2013 IEEE Int. Conf. Commun., pp. 3938-3942, 2013.

[265] S. Collins, R. Mulyawan, S. Rajbhandari, H. Chun, G. E. Faulkner, D. C. O’Brien, P. P. Manousiadis, D. A. Vithanage, G. A. Turnbull, and I. D. W. Samuel, "A Simple Wide Field of View Concentrator for Free Space Visible Light Communications," in IEEE Photonics Society Summer Topical Meeting Series, 2015, 2015, pp. 43-44.

[266] D. C. O’Brien, M. Katz, P. Wang, K. Kalliojarvi, S. Arnon, M. Matsumoto, R. Green, and S. Jivkova, "Short-range optical wireless communications," Wirel. World Res. Forum, vol. 1, no. 22, pp. 1-22, 2005.

[267] R. Ramirez-Iniguez and R. J. Green, "Optical antenna design for indoor optical wireless communication systems," vol. 18 , no. 3. pp. 229-245, 2005.

[268] S. Collins, D. C. O'Brien, and A. Watt, "High gain, wide field of view concentrator for optical communications," Opt. Lett., vol. 39, no. 7, pp. 1756-1759, 2014.

[269] G. Smestad, H. Ries, R. Winston, and E. Yablonovitch, "The thermodynamic limits of light concentrators," Sol. Energy Mater., vol. 21, no. 2-3, pp. 99-111, 1990.

[270] C. Tummeltshammer, A. Taylor, A. J. Kenyon, and I. Papakonstantinou, "Flexible and fluorophore-doped luminescent solar concentrators based on polydimethylsiloxane," Opt. Lett., vol. 41, no. 4, pp. 713-716, 2016.

[271] N. D. Bronstein, Y. Yao, L. Xu, E. O’Brien, A. S. Powers, V. E. Ferry, A. P. Alivisatos, and R. G. Nuzzo, “Quantum dot luminescent concentrator cavity exhibiting 30-fold concentration," ACS Photonics, vol. 2, no. 11, pp. 1576-1583, 2015.

[272] S. Rajbhandari, H. Chun, G. Faulkner, K. Cameron, A. V. N. Jalajakumari, R. Henderson, D. Tsonev, M. Ijaz, Z. Chen, H. Haas, E. Xie, J. J. D. McKendry, J. Herrnsdorf, E. Gu, M. D. Dawson, and D. O Brien, "High-speed integrated visible light communication system: Device constraints and design considerations," IEEE J. Sel. areas Commun., vol. 33, no. 9, pp. 17501757, 2015.

[273] J. M. Kahn, P. Djahani, A. G. Weisbin, K. T. Beh, A. P. Tang, R. You, P. Djahani, A. G. Weisbin, B. K. Teik, and A. P. Tang, "Imaging diversity receivers for high-speed infrared wireless communication," IEEE Commun. Mag., vol. 36, no. 12, pp. 88-94, 1998.

[274] T. Q. Wang, Y. A. Sekercioglu, and J. Armstrong, "Analysis of an optical wireless receiver using a hemispherical lens with application in MIMO visible light communications," J. Light. Technol., vol. 31, no. 11, pp. 1744-1754, 2013.

[275] B. Li, X. Lai, J. Wang, X. Liang, and C. Zhao, "Performance analysis of the imaging receivers using a hemispherical lens for Visible Light Communications," in 2013 International Conference on Wireless Communications and Signal Processing, 2013, pp. $1-5$.

[276] T. Chen, L. Liu, B. Tu, Z. Zheng, and W. Hu, "High-spatial-diversity imaging receiver using fisheye lens for indoor MIMO VLCs," IEEE Photonics Technol. Lett., vol. 26, no. 22, pp. 2260-2263, 2014.

[277] D. O’Brien, "Optical Multi-Input Multi-Output systems for short-range free-space data transmission," Communication Systems Networks and Digital Signal Processing (CSNDSP), 2010 7th International Symposium on. pp. 517-521, 2010.

[278] C. He, T. Q. Wang, A. Masum, and J. Armstrong, "Performance of optical receivers using photodetectors with different fields of view in an indoor cellular communication system," J. Light. Technol., vol. 33, no. 23, pp. 4957-4967, 2015.

[279] Y. Jiang, M. K. Varanasi, and J. Li, "Performance analysis of ZF and MMSE equalizers for MIMO systems: An in-depth study of the high SNR regime," IEEE Trans. Inf. Theory, vol. 57, no. 4, pp. 2008-2026, 2011.

[280] G. Ginis and J. M. Cioffi, "On the relation between V-BLAST and the GDFE," Commun. Lett. IEEE, vol. 5, no. 9, pp. 364-366, 2001.

[281] R. Fischer, J. Huber, and C. Windpassinger, "Signal processing in decision-feedback equalization of intersymbol-interference and multiple-input/multiple-output channels: a unified view," Signal Processing, vol. 83, no. 8, pp. 1633-1642, 2003.

[282] P. M. Butala, H. Elgala, and T. D. C. Little, "Performance of optical spatial modulation and spatial multiplexing with imaging receiver," in Wireless Communications and Networking Conference (WCNC), 2014 IEEE, 2014, pp. 394-399.

[283] E. Poves, W. Popoola, H. Haas, J. Thompson, and D. Cárdenas, "Experimental Results on the Performance of Optical Spatial Modulation Systems," in 2012 IEEE Vehicular Technology Conference (VTC Fall), 2012, pp. 1-5.

[284] D. O'Brien, "Multi-input multi-output (MIMO) indoor optical wireless communications," in 2009 Conference Record of the Forty-Third Asilomar Conference on Signals, Systems and Computers, 2009, pp. 1636-1639.

[285] P. A. Haigh, Z. Ghassemlooy, I. Papakonstantinou, F. Tedde, S. F. Tedde, O. Hayden, and S. Rajbhandari, "A MIMO-ANN system for increasing data rates in organic visible light communications systems," in Communications (ICC), 2013 IEEE International Conference on, 2013, pp. 5322-5327.

[286] A. H. Azhar, T. Tran, D. O. Brien, M. Ieee, and A. S. Overview, "Demonstration of high-speed data transmission using MIMOOFDM visible light communications," in GLOBECOM Workshops, 2010, pp. 1052-1056.

[287] Y. W. Yuanquan Wang and N. C. Nan Chi, "Indoor gigabit $2 \times 2$ imaging multiple-input-multiple-output visible light communication," Chinese Opt. Lett., vol. 12, no. 10, pp. 100603-100606, 2014.

[288] Z. Shen, T. Lan, L. Wang, and G. Ni, "Color demultiplexer using angularly multiplexed volume holograms as a receiver optical end for VLC based on RGB white LED,” Opt. Commun., vol. 333, pp. 139-145, 2014.

[289] Q. Chen, D. Chitnis, K. Walls, T. D. Drysdale, S. Collins, and D. R. S. Cumming, "CMOS photodetectors integrated with plasmonic color filters," IEEE Photonics Technol. Lett., vol. 24, no. 3, pp. 197-199, 2012.

[290] J.-Y. Sung, C.-W. Chow, and C.-H. Yeh, "Is blue optical filter necessary in high speed phosphor-based white light LED visible light communications?," Opt. Express, vol. 22, no. 17, pp. 20646-20651, 2014.

[291] H. Chun, S. Rajbhandari, G. Faulkner, and D. O’Brien, "Effectiveness of blue-filtering in WLED based indoor Visible light communication," 2014 3rd Int. Work. Opt. Wirel. Commun. IWOW 2014, pp. 60-64, 2014.

[292] N. J. Findlay, J. Bruckbauer, A. R. Inigo, B. Breig, S. Arumugam, D. J. Wallis, R. W. Martin, and P. J. Skabara, “An organic down-converting material for white-light emission from hybrid LEDs.," Adv. Mater., vol. 26, no. 43, pp. 7290-4, 2014.

[293] S. Jahangir, I. Pietzonka, M. Strassburg, and P. Bhattacharya, "Monolithic phosphor-free InGaN/GaN quantum dot wavelength converter white light emitting diodes," Appl. Phys. Lett., vol. 105, no. 11, p. 111117, Sep. 2014.

[294] N. Laurand, B. Guilhabert, J. McKendry, A. E. Kelly, B. Rae, D. Massoubre, Z. Gong, E. Gu, R. Henderson, and M. D. Dawson, "Colloidal quantum dot nanocomposites for visible wavelength conversion of modulated optical signals," Opt. Mater. Express, vol. 2, no. 3, p. 250, 2012.

[295] T. Kim, J. Kim, M.-S. Yang, S. Lee, Y. Park, U.-I. Chung, and Y. Cho, "Highly efficient yellow photoluminescence from \{11-22\} InGaN multiquantum-well grown on nanoscale pyramid structure,” Appl. Phys. Lett., vol. 97, no. 24, p. 241111, Dec. 2010.

[296] J. M. M. Santos, B. E. Jones, P. J. Schlosser, S. Watson, J. Herrnsdorf, B. Guilhabert, J. J. D. McKendry, J. De Jesus, T. A. Garcia, M. C. Tamargo, A. E. Kelly, J. E. Hastie, N. Laurand, and M. D. Dawson, "Hybrid GaN LED with capillary-bonded II-VI 
MQW color-converting membrane for visible light communications," Semicond. Sci. Technol., vol. 30, no. 3, p. $035012,2015$.

[297] M. T. Sajjad, P. P. Manousiadis, C. Orofino, D. Cortizo-Lacalle, A. L. Kanibolotsky, S. Rajbhandari, D. Amarasinghe, H. Chun, G. Faulkner, D. C. O'Brien, P. J. Skabara, G. A. Turnbull, and I. D. W. Samuel, "Fluorescent Red-Emitting BODIPY Oligofluorene Star-Shaped Molecules as a Color Converter Material for Visible Light Communications," Adv. Opt. Mater., vol. 3, no. 4, pp. 536-540, 2015.

[298] M. T. Sajjad, P. P. Manousiadis, H. Chun, D. A. Vithanage, S. Rajbhandari, A. L. Kanibolotsky, G. Faulkner, D. Obrien, P. J. Skabara, I. D. W. Samuel, and G. A. Turnbull, "Novel fast color-converter for visible light communication using a blend of conjugated polymers," ACS Photonics, vol. 2, no. 2, pp. 194-199, 2015.

[299] "MOST 2016 Technology report," 2016. [Online]. Available: http:/www.mostcooperation.com/publications/most-brochuresnewsletters/.

[300] R. C. A. Pitwon, K. Wang, J. Graham-Jones, I. Papakonstantinou, H. Baghsiahi, B. J. Offrein, R. Dangel, D. Milward, and D. R. Selviah, "FirstLight: Pluggable optical interconnect technologies for polymeric electro-optical printed circuit boards in data centers," J. Light. Technol., vol. 30, no. 21, pp. 3316-3329, 2012.

[301] M. U. Khan, J. Justice, J. Petäjä, T. Korhonen, A. Boersma, S. Wiegersma, M. Karppinen, and B. Corbett, "Multi-level single mode 2D polymer waveguide optical interconnects using nano-imprint lithography," Opt. Express, vol. 23, no. 11, p. 14630, 2015.

[302] Z. S. Rihawi, Z. Abdul Mutalip, R. J. Green, M. D. Higgins, and M. S. Leeson, "Free-Space Optical Communications in Vehicular Networks Using Rectangular Guiding Models," IEEE Photonics Technol. Lett., vol. 28, no. 13, pp. 1430-1433, 2016.

[303] S. H. Yu, O. Shih, H. M. Tsai, and R. D. Roberts, "Smart automotive lighting for vehicle safety," IEEE Commun. Mag., vol. 51, no. 12 , pp. 50-59, 2013.

[304] L. C. Wu and H. M. Tsai, "Modeling vehicle-to-vehicle visible light communication link duration with empirical data," 2013 IEEE Globecom Work. GC Wkshps 2013, pp. 1103-1109, 2013.

[305] P. Luo, Z. Ghassemlooy, H. Le Minh, E. Bentley, A. Burton, and X. Tang, "Fundamental analysis of a car to car visible light communication system," 2014 9th Int. Symp. Commun. Syst. Networks Digit. Signal Process. CSNDSP 2014, pp. 1011-1016, 2014.

[306] I. Takai, S. Ito, K. Yasutomi, K. Kagawa, M. Andoh, and S. Kawahito, "LED and CMOS image sensor based optical wireless communication system for automotive applications," IEEE Photonics J., vol. 5, no. 5, 2013.

[307] Y. H. Kim, W. A. Cahyadi, and Y. H. Chung, "Experimental Demonstration of VLC-Based Vehicle-to-Vehicle Communications Under Fog Conditions," IEEE Photonics J., vol. 7, no. 6, 2015.

[308] C. B. Liu, B. Sadeghi, and E. W. Knightly, "Enabling vehicular visible light communication (V2LC) networks," in Vanet'11, 2011, pp. 41-50.

[309] T. Nagura, T. Yamazato, M. Katayama, T. Yendo, T. Fujii, and H. Okada, "Tracking an LED array transmitter for visible light communications in the driving situation," in Proceedings of the 2010 7th International Symposium on Wireless Communication Systems, ISWCS'10, 2010, pp. 765-769.

[310] N. Kumar, "Visible Light Communication Based Traffic Information Broadcasting Systems," Int. J. Futur. Comput. Commun., vol. 3 , no. 1 , pp. 26-30, 2014 .

[311] H. C. N. Premachandra, T. Yendo, M. P. Tehrani, T. Yamazato, H. Okada, T. Fujii, and M. Tanimoto, "High-speed-camera image processing based LED traffic light detection for road-to-vehicle visible light communication," Intell. Veh. Symp. IV 2010 IEEE, pp. 793-798, 2010

[312] F. Hanson and S. Radic, "High bandwidth underwater optical communication,” Appl. Opt., vol. 47, no. 2, pp. 277-283, Jan. 2008.

[313] G. Cossu, R. Corsini, A. M. Khalid, S. Balestrino, A. Coppelli, A. Caiti, and E. Ciaramella, "Experimental demonstration of high speed underwater visible light communications," in 2013 2nd International Workshop on Optical Wireless Communications (IWOW), 2013, pp. 11-15.

[314] M. Doniec, I. Vasilescu, M. Chitre, C. Detweiler, M. Hoffmann-Kuhnt, and D. Rus, "AquaOptical: A lightweight device for highrate long-range underwater point-to-point communication," in OCEANS 2009, 2009, pp. 1-6.

[315] S. Arnon, "Underwater optical wireless communication network," Opt. Eng., vol. 49, no. 1, pp. 1-6, 2010.

[316] H. M. Oubei, J. R. Duran, B. Janjua, H.-Y. Wang, C.-T. Tsai, Y.-C. Chi, T. K. Ng, H.-C. Kuo, J.-H. He, M.-S. Alouini, G.-R. Lin, and B. S. Ooi, "4.8 Gbit/s 16-QAM-OFDM transmission based on compact 450-nm laser for underwater wireless optical communication," Opt. Express, vol. 23, no. 18, p. 23302, 2015.

[317] S. Arnon and D. Kedar, "Non-line-of-sight underwater optical wireless communication network," J. Opt. Soc. Am. A, vol. 26, no. 3, pp. 530-539, Feb. 2009.

[318] S. P. Najda, P. Perlin, T. Suski, L. Marona, M. Boćkowski, M. Leszczyński, P. Wisniewski, R. Czernecki, R. Kucharski, G. Targowski, S. Watson, and A. E. Kelly, "Free-space and underwater GHz data transmission using AlGaInN laser diode technology," 2016, p. 983309.

[319] J. Armstrong, Y. Sekercioglu Ahmet, and A. Neild, "Visible light positioning: A roadmap for international standardization," IEEE Commun. Mag., vol. 51, no. 12, pp. 68-73, 2013.

[320] D. Iturralde, C. Azurdia-Meza, N. Krommenacker, I. Soto, Z. Ghassemlooy, and N. Becerra, "A new location system for an underground mining environment using visible light communications," in 2014 9th International Symposium on Communication Systems, Networks \& Digital Sign (CSNDSP), 2014, pp. 1165-1169.

[321] S.-Y. Jung, C.-K. Choi, S. H. Heo, S. R. Lee, and C.-S. Park, "Received signal strength ratio based optical wireless indoor localization using light emitting diodes for illumination," in IEEE International Conference on Consumer Electronics (ICCE), 2013, pp. 63-64.

[322] W. Zhang, M. I. S. Chowdhury, and M. Kavehrad, "Asynchronous indoor positioning system based on visible light communications," Opt. Eng., vol. 53, no. 4, p. 045105, 2014.

[323] Z. Zhou, M. Kavehrad, and P. Deng, "Indoor positioning algorithm using light-emitting diode visible light communications," Opt. Eng., vol. 51, no. 8, pp. 1-7, 2012.

[324] T.-H. Do and M. Yoo, “An in-Depth Survey of Visible Light Communication Based Positioning Systems,” Sensors, vol. 16, no. 5, p. 678,2016

[325] I. Vol, J. Ivkovi, N. S. Technical, and M. Pupin, "Analysis of Visible Light Communication System for Implementation in Sensor Networks," INFOTEH-JAHORINA, vol. 15, no. March, pp. 274-279, 2016.

[326] K. Warmerdam, A. Pandharipande, and D. Caicedo, "Connectivity in IoT indoor lighting systems with visible light communications," 2015 IEEE Online Conf. Green Commun. OnlineGreenComm 2015, pp. 47-52, 2016.

[327] L. T. Dung, S. Jo, and B. An, "Demonstration of Low-Complexity LED-to-LED Two-way Visible Light Communication System," Ieee Is3C 2016, no. April, 2016.

[328] C. Wang, Z. Zhang, M. Zhang, and H. Zhu, "Visible light communication application scenarios based on Android smart devices' LED lamp," Photonic Netw. Commun., pp. 1-5, Feb. 2016. 
[329] B. Zhang, K. Ren, G. Xing, X. Fu, and C. Wang, "SBVLC: Secure barcode-based visible light communication for smartphones," in Proceedings - IEEE INFOCOM, 2014, pp. 2661-2669.

[330] "Global Visible Light Communication (VLC)/Li-Fi Technology Market worth \$6,138.02 Million by 2018," MarketsandMarkets, 2013. [Online]. Available: http://www.prnewswire.com/news-releases/global-visible-light-communication-vlcli-fi-technologymarket-worth-613802-million-by-2018-186272641.html. [Accessed: 21-Sep-2016].

[331] A. Jovicic, L. Junyi, and T. Richardson, "Visible light communication: opportunities, challenges and the path to market," Commun. Mag. IEEE, vol. 51, no. 12, pp. 26-32, 2013.

[332] "Carrefour guides shoppers to in-store discounts via the ceiling lights." [Online]. Available: http://luxreview.com/article/2015/05/carrefour-guides-shoppers-to-in-store-discounts-via-the-ceiling-lights.

[333] R. Mesleh, H. Elgala, and H. Haas, "LED Nonlinearity Mitigation Techniques in Optical Wireless OFDM Communication Systems," J. Opt. Commun. Netw., vol. 4, no. 11, p. 865, Nov. 2012.

[334] Z. Wang, Q. Wang, S. Chen, and L. Hanzo, "An adaptive scaling and biasing scheme for OFDM-based visible light communication systems,” Opt. Express, vol. 22, no. 10, p. 12707, 2014. 